University of Nebraska - Lincoln

DigitalCommons@University of Nebraska - Lincoln

U.S. Air Force Research

U.S. Department of Defense

2014

Reduced order unsteady aerodynamic modeling for stability and control analysis using computational fluid dynamics

Mehdi Ghoreyshi

U.S. Air Force Academy

Adam Jirasek

U.S. Air Force Academy

Russell M. Cummings

USAF Academy

Follow this and additional works at: http:// digitalcommons.unl.edu/usafresearch

Ghoreyshi, Mehdi; Jirasek, Adam; and Cummings, Russell M., "Reduced order unsteady aerodynamic modeling for stability and control analysis using computational fluid dynamics" (2014). U.S. Air Force Research. 57.

http://digitalcommons.unl.edu/usafresearch/57

This Article is brought to you for free and open access by the U.S. Department of Defense at DigitalCommons@University of Nebraska - Lincoln. It has been accepted for inclusion in U.S. Air Force Research by an authorized administrator of DigitalCommons@University of Nebraska - Lincoln. 


\title{
Reduced order unsteady aerodynamic modeling for stability and control analysis using computational fluid dynamics
}

\author{
Mehdi Ghoreyshi*, Adam Jirásek, Russell M. Cummings \\ High Performance Computing Research Center, U.S. Air Force Academy, USAF Academy, CO 80840-6400, USA
}

\section{A R T I C L E I N F O}

\section{Article history:}

Received 12 August 2014

Accepted 2 September 2014

Available online 3 October 2014

Keywords:

Unsteady aerodynamics

Computational fluid dynamics

Reduced order model

Indicial functions

Radial basis functions

Volterra functions

\begin{abstract}
A B S T R A C T
Recent advances and challenges in the generation of reduced order aerodynamic models using computational fluid dynamics are presented. The models reviewed are those that can be used for aircraft stability and control analysis and include linear and nonlinear indicial response methods, Volterra theory, radial basis functions, and a surrogate-based recurrence framework. The challenges associated with identification of unknowns for each of the reduced order methods are addressed. A range of test cases, from airfoils to full aircraft, have been used to evaluate and validate the reduced order methods. The motions have different amplitudes and reduced frequencies and could start from different flight conditions including those in the transonic speed range. Overall, these reduced order models help to produce accurate predictions for a wide range of motions, but with the advantage that model predictions require orders of magnitude less time to evaluate once the model is created.
\end{abstract}

Published by Elsevier Ltd.

\section{Contents}

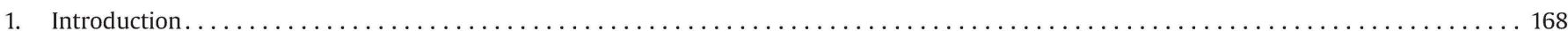

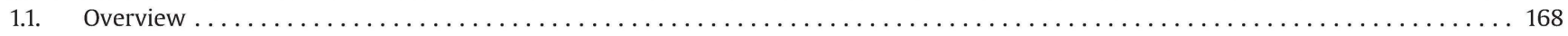

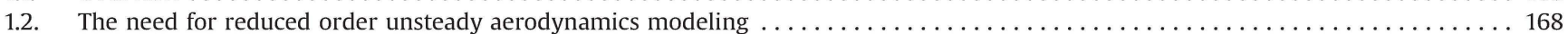

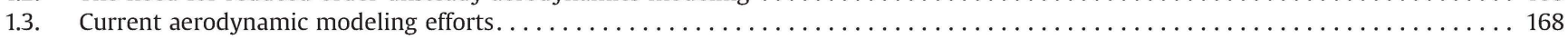

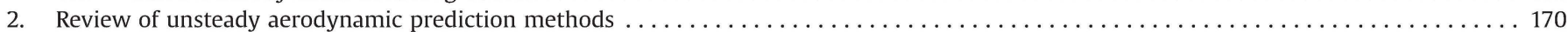

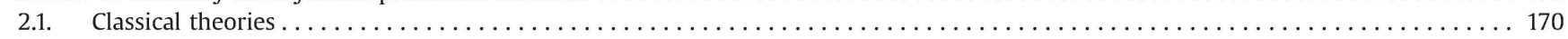

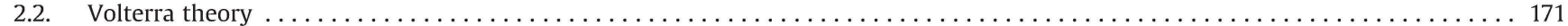

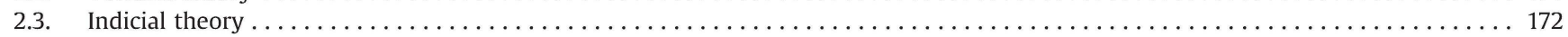

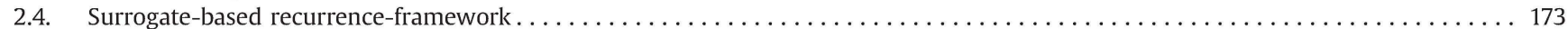

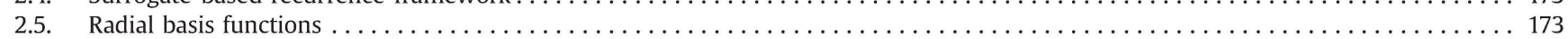

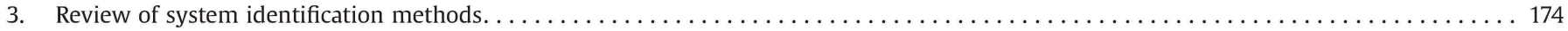

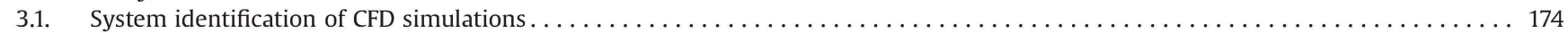

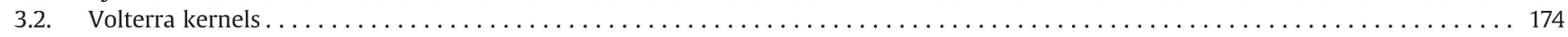

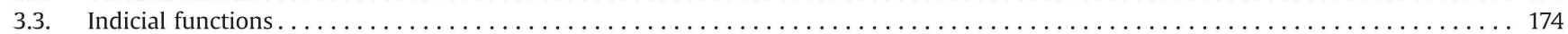

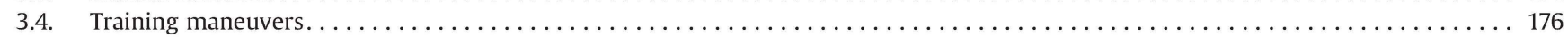

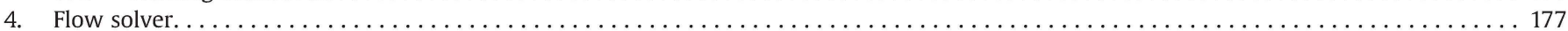

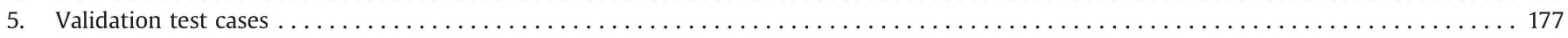

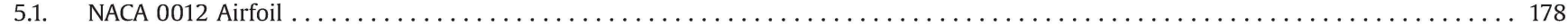

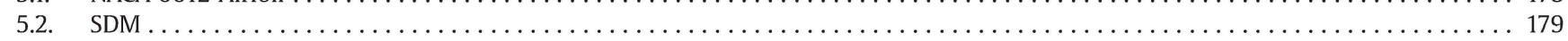

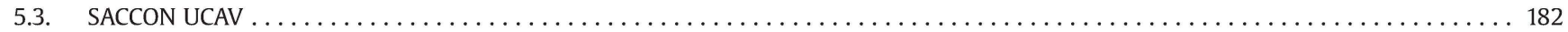

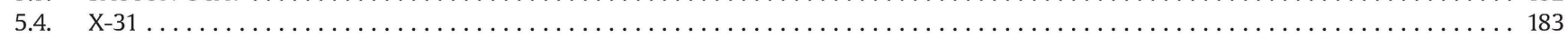

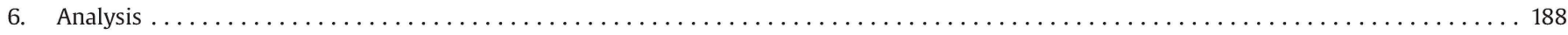

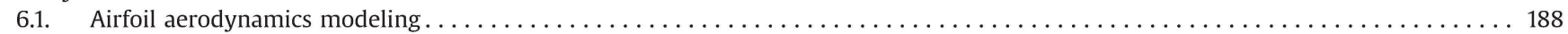

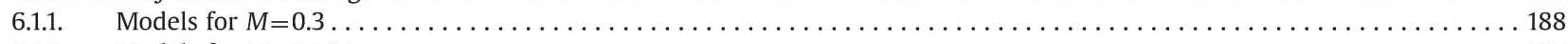

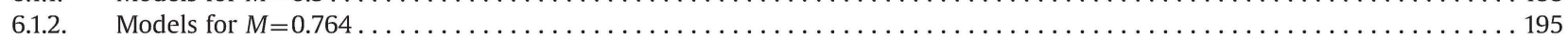

\footnotetext{
* Corresponding author.

E-mail addresses: Mehdi.Ghoreyshi@usafa.edu (M. Ghoreyshi), Adam.jirasek@gmail.com (A. Jirásek), Russ.Cummings@usafa.edu (R.M. Cummings).
} 


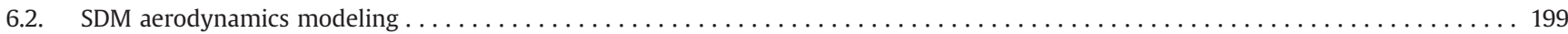

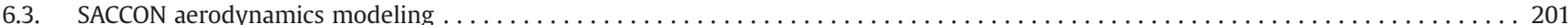

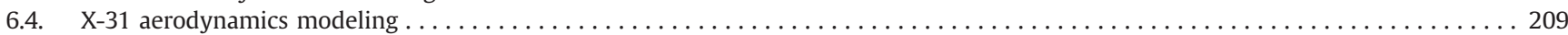

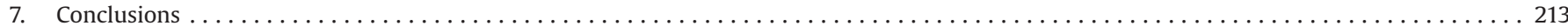

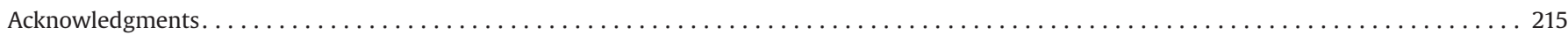

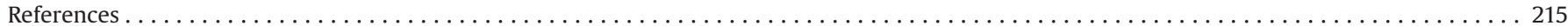

\section{Introduction}

\subsection{Overview}

The understanding and prediction of unsteady nonlinear aerodynamics attach great importance to advance aircraft aeroelasticity and flight dynamics. Maneuvering flight at high angles of attack, fast motion rates, and gusty wind conditions, as well as sudden control surface deflections and flutter phenomenon, all are examples of unsteady flight conditions [1]. The unsteady aerodynamic forces and moments in these conditions can have a significant effect on aircraft aeroelastic and dynamic stability characteristics [2]. In particular, flow hysteresis in a rapid maneuvering fighter can lead to pronounced time lags in the increase and decline of aerodynamic loads with respect to the flow field changes $[3,4]$. The main sources of aerodynamic nonlinearities are shock wave motions and separated flows which can generate limit cycle oscillations and have a significant impact on aircraft performance and stability [5]. An aerodynamic model that can accurately predict the nonlinear and unsteady airloads of a maneuvering aircraft can improve the accuracy of the structural analysis and flight control design. This translates into reduced project risk and enhanced analysis of system performance prior to first flight.

A wide range of mathematical models have been developed to represent the unsteady aerodynamic loads for use in aircraft stability and control $(\mathrm{S} \& \mathrm{C})$ analysis, including classical linearized models using rotary and unsteady aerodynamic derivatives (the $\dot{\alpha}$ terms) [6]. The unsteady derivatives of static and weakly nonlinear systems can be determined by a Taylor series expansion about given equilibrium flight conditions. However, these derivatives are not suitable for analysis of a high performance aircraft with highly nonlinear and unsteady aerodynamics $[7,8]$. There are also only limited experimental measurements available to determine the effects of unsteady flow on the aerodynamic forces and moments acting on an aircraft. This is mainly due to the complexity of unsteady flow and the limitations of existing test facilities [8]. During the past decade, efforts have been made at formulating techniques that predict continuous and discrete-time aerodynamic responses using Computational Fluid Dynamics (CFD) $[9,10]$. These recent advances have led to the generation of unsteady nonlinear aerodynamic models for six-degree-of-freedom (6DoF) aircraft maneuvers with moving control surfaces. This work reviews the current state-of-the-art in unsteady aerodynamic modeling for use in S\&C and discusses current challenges for these modeling approaches.

\subsection{The need for reduced order unsteady aerodynamics modeling}

With advances in computing techniques, one straightforward way to predict flow-induced nonlinearities is to develop a fullorder model (often called a time-marching CFD solution) based on time integration of the structural and aerodynamic equations [11-13]. However, creating a full-order model for S\&C is a computationally expensive approach and is impractical from a designer's point of view because it requires a large number of computations for different values of motion frequency and amplitude $[14,15]$. Also, this approach makes the solution of the aircraft equations of motion an infinite-dimensional problem, where the current states depend on the evolution of previous states at infinitely many points in time [16]. It is therefore desirable to use a relatively simple but accurate approximation for the unsteady aerodynamics by using a Reduced Order Model (ROM) that allows describing the unsteady flow in the form of a small number of spatial/temporal modes (typically less than one hundred) compared with the very large number of grid points in the full-order model (on the order of 5-50 million or more) [17-19]. The ROM can then predict the responses to an arbitrary input much faster than computing the input in a full CFD solution [20].

The creation of ROMs using CFD is an area of active research. These models can be classified into nonparametric and parametric models depending on the identification methods used [21,22]. The parametric models provide a structure for representing aerodynamic forces and moments in the aircraft equations of motion. Nonparametric models, on the other hand, are concerned with the measured input-output behavior of the aircraft dynamics [21]. ROMs can also be classified into time domain and frequency domain approaches [9]. While time-domain models are necessary for rotary wing applications and predicting responses to a timedependent input, the formulation in frequency-domain is required for determination of flutter instability boundary [23]. Some popular ROMs are indicial response methods [24-27], proper orthogonal decomposition (POD) [28,29], Volterra theory [22,30-32], surrogate-based recurrence framework (SBRF) [33,34], radial basis functions (RBF) [35-37] and state-space modeling [38,39]. Notice that some of these models, such as indicial response methods, Volterra theory, and POD analysis, can be performed either in the time or frequency domain. Recent applications of POD methods has been reviewed by Lucia et al. [9] and therefore these methods are not discussed here. Also, the state space model proposed by Goman and Khrabrov [38] uses experimental data to estimate the model unknowns. There are only a few published studies of this model using CFD and therefore the model is also not discussed. This paper reviews the recent development and applications of Volterra theory, linear and nonlinear indicial response methods, RBF, and a SBRF model using CFD data. These models were selected because they have been used or can be used in the aircraft $S \& C$ analysis.

\subsection{Current aerodynamic modeling efforts}

Early numerical models of unsteady aerodynamics were mostly developed for flows in cascades and turbomachineries and were based on the Fourier series decomposition of the inputs and responses [40-44]. These models can be performed either in the time or frequency domain. However, for time-periodic flows, such as flows about helicopter rotors and flows in turbomachinery, the frequency-domain models can dramatically reduce the computational time compared with time-domain models [45]. The recent modeling efforts of the Fourier-based methods were published in a special section of the International Journal of Computational Fluid Dynamics [45-50] and have also been reviewed by He [51]. 


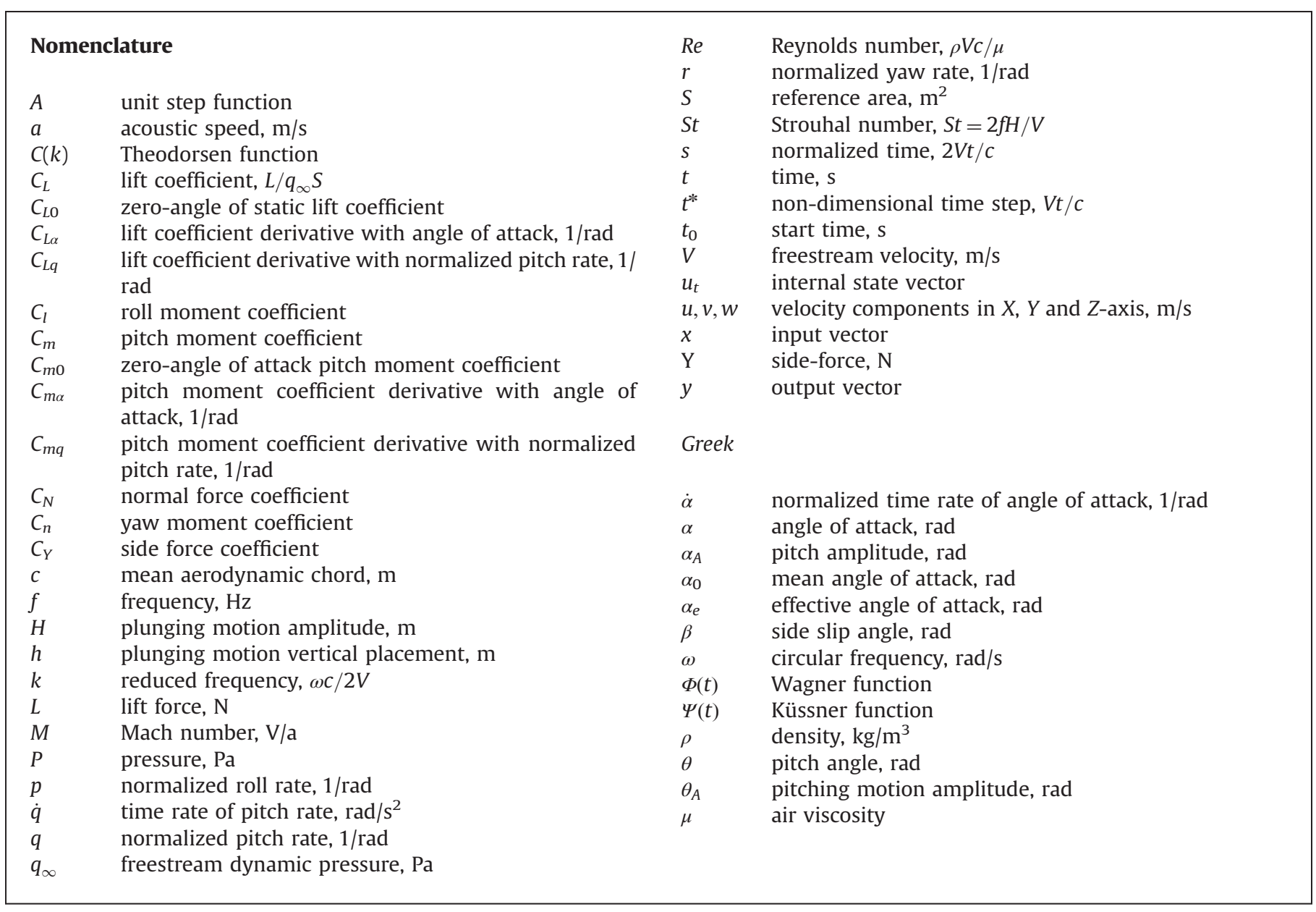

For fixed wing aircraft, aerodynamic damping derivatives are usually used to represent unsteady effects. However, a derivativebased model breaks down for modern combat aircraft undergoing agile maneuver at high angles of attack [8]. A wide range of efforts are emerging to extend the application of these models to advanced fighter aircraft. For example, the work of Morton and his colleagues [52-55] at the Air Force SEEK EAGLE Office (AFSEO) involves aerodynamic modeling for aircraft flight simulation, control system design, and optimization. They begin with a global nonlinear parameter modeling technique proposed by Morelli [56] that describes the functional dependence between a motion and its computed aerodynamic response in terms of force and moment coefficients. They then attempt to find a new model which has adequate complexity to capture the aerodynamic nonlinearities while keeping the number of terms in the model low. The latter requirement improves the ability to identify model parameters, resulting in a more accurate model with good predictive capabilities. The model unknowns are then identified using CFD simulations of some training maneuvers. These techniques have been incorporated into the Department of Defense (DoD) CREATE-AV code, Kestrel.

Various reduced order unsteady aerodynamic models have also been used at the University of Michigan. Skujins and Cesnik [57-59], for example, predicted unsteady loads due to the oscillation of multiple vehicle elastic modes using convolution of modal step responses along with a correction factor which takes into account different oscillation amplitudes, fight conditions, and nonlinear aerodynamic effects due to multi-modal oscillations. Glaz et al. [33] also described a reduced-order nonlinear unsteady aerodynamic modeling approach suitable for analyzing pitching/plunging airfoils subject to fixed or time-varying freestream Mach numbers.
The reduced-order model uses Kriging surrogates to account for flow nonlinearities and recurrence solutions to account for timehistory effects associated with unsteadiness. They showed that the model can accurately predict time-varying freestream Mach number effects and is therefore applicable to rotary wing and fixed wing applications. Later, they used this modeling approach for the prediction of unsteady lift, pitch moment, and drag of an airfoil pitching/plunging under dynamic stall conditions [60].

The aerodynamic modeling studies at Duke University are mainly focused on frequency domain techniques of Harmonic Balance (HB), POD-based models, and Volterra series, all applied to different aeromechanics problems including flutter, forced response, limit cycle oscillations, multistage flows, and non-synchronous vibrations. The HB approach assumes that unsteady flows are temporally and spatially periodic and then flow variables are represented by a Fourier series in time with spatially varying coefficients [61]. The full data at each grid point is then reduced to $2 N+1$ coefficients, where $N$ is the number of harmonics retained in the Fourier series representation of the flow. The two other models used at Duke are based on POD and Volterra series. For example, Thomas et al. [62] applied POD-based models to an inviscid flow transonic wing configuration and reduced the CFD model with over three quarters of a million degrees of freedom to a system with just a few dozen degrees of freedom, while still retaining the accuracy of the unsteady aerodynamics of the full system representation. Also, the Volterra series approach by Balajewicz and his colleagues $[63,64]$ was investigated for flutter boundary and LCO analysis. They used a pseudo-inverse approach and training inputs from the full-order system to identify Volterra kernels.

At Stanford University, POD methods have been used for unsteady aerodynamic analysis by Farhat and his colleagues. 
For example, Lieu and Farhat [65] proposed a ROM based on POD for evaluating aeroelastic frequencies and damping ratio coefficients of the F-16 aircraft. They extended previous ROMs to a multiple state variable model by including the variation of Mach number and angle of attack flight conditions. Jameson and his colleagues at Stanford have also applied HB techniques to accelerate the convergence of unsteady flows to a periodic steady state $[66,67]$. The HB techniques have also been successfully tested by Badcock and his colleagues at the University of Liverpool $[68,69]$.

Time domain based neural networks have also been used to model unsteady aerodynamics [70-73]. Olcer et al. [70] used neural networks for modeling the unsteady aerodynamic responses to a trailing edge flap deflection and to extract a linear time-invariant model of the flap actuator. Marques and Anderson [71] used a temporal neural network to approximate the unsteady lift and pitch moment of a two dimensional airfoil with changes in angle of attack in the transonic regime. Faller and Schreck [73] also used a neural network to predict the time-dependent surface pressures of a pitching wing. The network inputs included the instantaneous pitch angle, angular velocity, and the initial surface pressure coefficients at $t_{0}$. The network output then predicted the surface pressures at time $t_{0}+\Delta t$. These predictions were fed back as the input to the network to predict surface pressures at the next time. This process continues in time until it reaches some final time.

Some other works to be mentioned include the studies at the University of Maryland [74-76,76], the work of Raveh [77,78,20] at the Israel Institute of Technology, the work of Beran [9,79] at the Air Force Research Laboratory, the work of Silva [30,22] at NASA Langley, and the work of Murman [80,81] at NASA Ames. Finally, researchers at the U.S. Air Force Academy (USAFA) have made a strong effort toward the development of unsteady aerodynamic reduced order models applied to flight mechanics and flow control.

\section{Review of unsteady aerodynamic prediction methods}

The input to, and output from, a model are defined by $x(t)$ and $y$ $(t)$, respectively. Note that for a dynamic system (a system with memory) $y$ is said to be a functional of $x$, not the function of $x[82]$.

\subsection{Classical theories}

Classical solutions have been obtained for the airfoil lift and pitch moment responses to harmonic pitching and plunging motions and wind gusts by Theodorsen [83], von Karman [84], Küssner [85], Sears [86], and Wagner [87]. Pitching and plunging oscillations are shown in Fig. 1 and are described by

$\theta(t)=\theta_{A} \cos \left(\omega_{\theta} t\right), \quad h(t)=H \cos \left(\omega_{h} t\right)$

where $\theta_{A}$ and $\omega_{\theta}$ are the pitch amplitude and rotational velocity, respectively; $h$ is the vertical placement of the airfoil, $H$ is the plunge amplitude, and $\omega_{h}$ denotes the oscillatory frequency. The reduced frequencies of $k_{\theta}$ and $k_{h}$ are defined as $\omega_{\theta} c / 2 V$ and $\omega_{h} c / 2 V$, where $c$ is the airfoil chord and $V$ is the freestream velocity. The plunge motion has no rotation but the angle of attack changes due to the

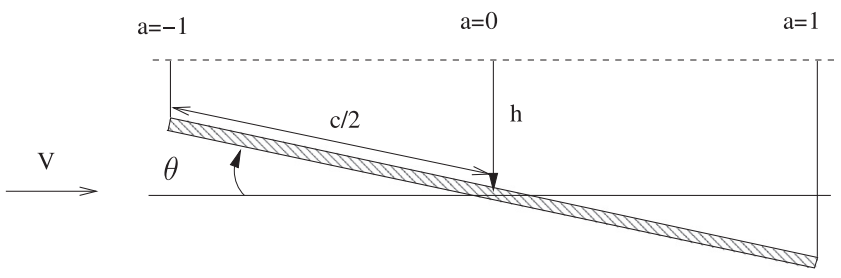

Fig. 1. Airfoil pitch and plunge maneuvers. vertical displacements of the airfoil; this angle is named the "effective angle of attack", which is denoted by $\alpha_{e}$ and is defined as

$\alpha_{e}=\tan ^{-1}\left(\frac{\dot{h}}{V}\right)$

The maximum effective angle of attack for a plunge oscillation starting at zero degrees angle of attack is determined by the Strouhal number (defined as $S t=2 f H / V)$ such that $\alpha_{e}^{\max }=\tan ^{-1}(\pi S t)$. The Strouhal number is a dimensionless number that is useful for describing unsteady flow of oscillating geometries [16]. There are a number of theories to predict the aerodynamic loads of pitching and plunging motions. Wagner [87] developed a theory based on potential flow, and detailed the unsteady lift coefficient of an airfoil undergoing a plunging motion as

$C_{L}=2 \pi \int_{0}^{t} \Phi(t-\tau) \dot{\alpha}_{e}(\tau) d \tau$

where $\dot{\alpha}_{e}=d \alpha_{e} / d t ; C_{L}$ is the lift force coefficient and $\Phi(t)$ is Wagner function with its exact values known in terms of Bessel functions. The Wagner function was approximated in non-dimensional time by a two-pole exponential function as [75]

$\Phi(s)=1-0.165 \exp (-0.0455 s)-0.335 \exp (-0.3 s)$

where $s=2 V t / c$ is a non-dimensional time. Wagner's approximation function is shown graphically in Fig. 2(a) which shows that the unsteady lift increases asymptotically in time to reach the quasisteady value at one degree angle of attack, while it reaches almost $90 \%$ of this value after traveling a distance equivalent to 15 semichords [88].

Lomax [89] used the linearized Euler equations to derive exact initial values of Wagner function for a flat-plate airfoil as

$\Theta(s)=\frac{1}{2 \pi} \frac{4}{M}\left(1-\frac{1-M}{2 M} s\right)$

where $M$ is the Mach number. Note that Lomax's theory is valid only for the initial time of plunging motions. Theodorsen [83], assuming potential flow, used the method of conformal mapping to derive the solution of unsteady aerodynamic loads of a thin airfoil undergoing small pitching and plunging oscillations. His theory was expanded by Hodges and Pierce [90] for the lift and the pitch moment about the quarter-chord as

$C_{L}=2 \pi\left[\frac{\dot{h}}{V}+\theta+\frac{c \dot{\theta}}{2 V}\right] C(k)+\frac{\pi}{2}\left(\frac{c \ddot{h}}{V^{2}}+\frac{c \dot{\theta}}{V}-\frac{a c^{2} \ddot{\theta}}{V^{2}}\right)$

$C_{m}=-\frac{\pi}{2}\left[\frac{1}{2} \frac{c \ddot{h}}{V^{2}}+\frac{\dot{c} \theta}{V}+\left(\frac{1}{8}-\frac{a}{2}\right) \frac{c^{2} \ddot{\theta}}{V^{2}}\right]$

where $a$ is the pitch axis location with respect to the half-chord as shown in Fig. 1. In this definition, the leading edge and trailing edge correspond to values of $a=-1$ and $a=1$, respectively. In Eq. (6), the first of the two terms in the lift shows the circulatory lift and the second term shows non-circulatory effects. Noncirculatory effects, also called added mass, are generated from motion accelerations. Circulatory effects, on the other hand, are generated from the differences in the velocity on the upper and lower surfaces of the airfoil. For the airfoil pitch moment about the quarter-chord the non-circulatory terms are usually negligible.

The effective angle of attack is defined as

$\alpha_{e}=C(k)\left[\frac{\dot{h}}{V}+\theta+\frac{c \dot{\theta}}{2 V}\right]$

where $C(k)$ is called Theodorsen function and is given by

$C(k)=\frac{H_{1}^{(2)}(k)}{H_{1}^{(2)}(k)+i H_{0}^{(2)}(k)}$ 
a

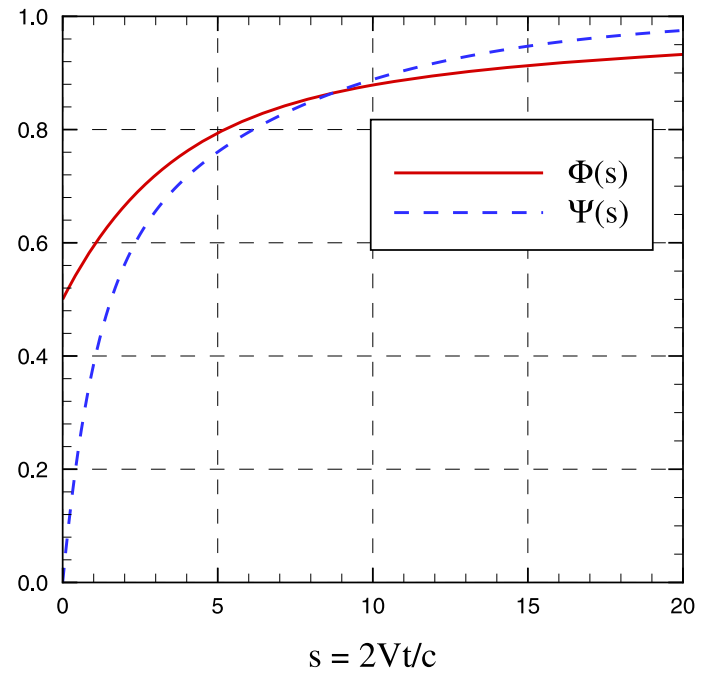

b

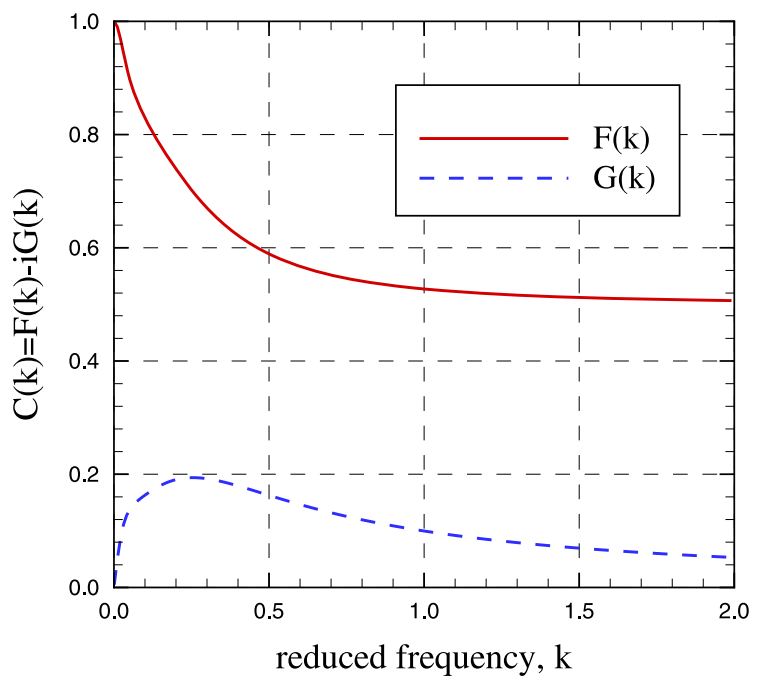

Fig. 2. Unsteady aerodynamic force functions for incompressible flow over an airfoil. (a) Wagner and Küssner functions and (b) Theodorsen's functions.

where $H_{0}$ and $H_{1}$ are Hankel functions of the first and the second kind. The Theodorsen function is real and equals one for the steady case (i.e. $k=0$ ) and includes an imaginary and a real part for a moving airfoil. These parts are expressed as $C(k)=F(k)-i G(k)$ and are shown in Fig. 2(b) for reduced frequencies up to two. The figure shows that $F(k)$ decreases with reduced frequency, thus the phase lag between the motion variables and unsteady loads is increased. The Theodorsen function is related to the Wagner function as [91]

$C(k)=(i k) \int_{0}^{\infty} \Phi(\tau) e^{-i k \tau} d \tau$

and is approximated as

$C(k)=1-\frac{0.165(i k)}{i k+0.0455}-\frac{0.335(i k)}{i k+0.3}$

Also, Küssner [85] defined a "Wake Vortex Function", later named the Küssner function, $\Psi(t)$, to find the unsteady lift of an airfoil in response to a sharp-edged gust with an upwash velocity of $\omega_{\mathrm{g}}$ given as

$C_{L}=2 \pi / V\left(\omega_{g}(0) \Psi(s)+\int_{0}^{s} \Psi(s-\sigma) \dot{\omega}_{g}(\sigma) d \sigma\right)$

where $\dot{\omega}_{g}=d \omega_{g} / d t$. The Küssner function can be related to Wagner function using a Fourier-integral and was approximated in terms of the Jones exponential expansion as

$\Psi(s)=1-0.5 \exp (-0.15 s)-0.5 \exp (-s)$

Fig. 2(a) compares Küssner and Wagner functions; it shows that Küssner function is zero at $s=0$ and reaches unity for very large $s$ values. While Küssner function predicts the responses to a sharpedged gust, Sears [86] derived the frequency response to a sinusoidal gust as well. Note that these theories are limited only to potential flow and small-amplitude airfoil motions. For a modern combat aircraft maneuvering at high angles of attack, a full nonlinear, large-amplitude, unsteady aerodynamic model is required.

\subsection{Volterra theory}

The Volterra theory [92] is considered one of the most important tools for the representation of nonlinear dynamic systems in the time and frequency domains [93]. The approach is a functional Taylor series expansion for dynamic systems (often called functional expansions) [94], therefore, the limitations of
Taylor series also apply to the Volterra series [94], e.g. the Volterra series typically is accurate for weak nonlinearities [95]. The theory of functional expansions was first introduced by Volterra in a book published in 1930 [92]. This work and the further developments by others (for example [96-98]) have been extensively used in electrical and biological systems engineering [99-103]. Recently, there is an increasing interest in using Volterra series in the field of nonlinear unsteady aerodynamic loads modeling [10,104].

For a dynamic system, the output in a given time instant of $t_{1}$ depends on the input values over some finite interval [82], i.e.

$y\left(t_{1}\right)=F\left[x(t), t_{1}-T \leq t \leq t_{1}\right]$

where $F$ is a continues functional of the input. Volterra used a $p$-th order series to estimate the values of $y$ in a given time as

$y(t)=\mathbf{H}_{0}+\sum_{i=1}^{p} \mathbf{H}_{i}(x(t))$

where $\mathbf{H}_{0}$ is the steady-state term and is constant; $\mathbf{H}_{i}$ represents the $i$-th order Volterra operator. In Eq. (15), $p$ may be indefinite to make sure that $y$ converges to the exact solution. However, it has been shown that for a time-invariant system, the output of $y$ for each $t \geq 0$ can be approximated by the right side of Eq. (15) for sufficiently large but finite values of $p$ as well [82]. For such a system, the Volterra operator is defined as an $i$-fold convolution between the input, $x(t)$, and the $i$-th order Volterra kernel, $H_{i}$, i.e.

$\mathbf{H}_{i}(x(t))=\int_{-\infty}^{t} \ldots \int_{-\infty}^{t} H_{i}\left(t-\tau_{1}, t-\tau_{2}, \ldots, t-\tau_{i}\right) \prod_{n=1}^{i} x\left(\tau_{n}\right) d \tau_{n}$

and using the commutative law, it can also be written as

$\mathbf{H}_{i}(x(t))=\mathbf{H}_{0}+\int_{-\infty}^{t} \ldots \int_{-\infty}^{t} H_{i}\left(\tau_{1}, \tau_{2}, \ldots, \tau_{i}\right) \prod_{n=1}^{i} x\left(t-\tau_{n}\right) d \tau_{n}$

Korenberg and Hunter [82] mentioned some Volterra kernel characteristics: (1) the zero order Volterra kernel of $\mathbf{H}_{0}$ is a constant value and found from the zero-input response of the system, (2) for a casual system the kernel of $H_{i}\left(\tau_{1}, \tau_{2}, \ldots, \tau_{i}\right)=0$ when any of $\tau_{1}, \tau_{2}, \ldots, \tau_{i}$ is less than zero, and the lower limits of the integrals can be set to zero, and (3) it can be assumed that each kernel is symmetric with respect to any permutation of $\tau_{1}, \tau_{2}, \ldots, \tau_{i}$. The Volterra series approximation can be used to represent any continuous-time, time-invariant, finite memory, and single-input and single-output system. The nonlinear unsteady aerodynamics is considered such a system (ignoring those hysteresis effects that make the solution infinite-memory) except that it is a multi-input 
and multi-output system. For a forced oscillation about the pitch axis, the output function $y$ is defined as $y=C_{L, D, m}(t)$ (corresponding to lift, drag, and pitch moment) and the input vector includes

$\mathbf{x}(t)=(\alpha(t), q(t), \dot{q}(t))$

where $q$ and $\dot{q}$ denote normalized pitch rate and its first time derivative, respectively. Classical Volterra series cannot properly identify any nonlinear cross-coupling effects between multiple input parameters [5]. Balajewicz et al. [105] applied a multi-input Volterra theory to the weakly nonlinear aerodynamic modeling of NACA 0012 airfoil under pitching and plunging motions. Their results showed a significant improvement in the accuracy over a single-input Volterra model. Also, Ghoreyshi et al. [34] proposed a multi-input Volterra series for aerodynamics prediction of the $\mathrm{X}-31$ aircraft pitching in the transonic speed range. This series is written as

$y(t)=\Psi\left(x_{1}(t), x_{2}(t), \ldots, x_{m}(t)\right)=\sum_{i=1}^{p} \mathbf{H}_{i}^{m}$

where the term $\mathbf{H}_{i}^{m}$ is the multi-input Volterra operator defined as a $m^{p}$-fold summation of $p$-fold convolution integrals between the inputs and the $p$-th order multi-input Volterra kernels [105]. The output response up to second order is rewritten as

$$
\begin{aligned}
y(t)= & \mathbf{H}_{0}+\sum_{j=1}^{m} \int_{-\infty}^{t} H_{1}^{x_{j}}(t-\tau) x_{j}(\tau) d \tau \\
& +\sum_{j_{1}=1}^{m} \sum_{j_{2}=1}^{m} \int_{-\infty}^{t} \int_{-\infty}^{t} H_{2}^{x_{j_{1}}, x_{j_{2}}}\left(t-\tau_{1}, t-\tau_{2}\right) x_{j_{1}}\left(\tau_{1}\right) x_{j_{2}}\left(\tau_{2}\right) d \tau_{1} d \tau_{2} \\
& +\mathcal{O}\left(|x|^{3}\right)
\end{aligned}
$$

Note that the superscripts in Eq. (20) identify to which inputs the kernel corresponds, for example, the second-order kernel $H_{2}^{x_{j_{1}}, x_{j_{3}}}$ correlates the inputs $x_{j_{1}}$ and $x_{j_{3}}$. Also, it should be noted that the second and higher-order kernels are symmetric with respect to the arguments, $H_{2}^{x_{1}, x_{j_{3}}}=H_{2}^{x_{j_{3}}, x_{j_{1}}}$. To estimate the output of $y$ using a Volterra series, the kernels of $\mathbf{H}_{i}^{m}$ must be determined using a system identification method. Unfortunately, the kernels of a nonlinear dynamic system (third- and higher-order kernels) are very difficult to compute [22].

\subsection{Indicial theory}

The Heaviside response to a unit step change in input function (angle-of-attack, pitch rate, etc.) is the so-called indicial response. Assuming a linear relationship between the input function and the output, a linear ROM is defined as the convolution (or Duhamel's superposition [106]) of the indicial response with the derivative of the forcing function [107]

$y(t)=x(0) A(t)+\int_{0}^{t} A(t-\tau) \dot{x}(\tau) d \tau$

where $\dot{x}=d x / d t ; A$ represents the unit response, or indicial response, of the system and $t-\tau$ is elapsed time; $x$ is input or forcing parameter and $x(0)$ shows input value at time zero.

The indicial response functions are used as a fundamental approach to represent the unsteady aerodynamic loads. For aerodynamic loads modeling, the mathematical models are detailed by Tobak et al. [25,26] and Reisenthel et al. [108,109]. It is assumed that lift and pitch moment depend on angle of attack and pitch rate. Indicial responses due to a unit step change in angle of attack, $\alpha$, and normalized pitch rate, $q$, are denoted by $C_{j_{\alpha}}$ and $C_{j_{q}}$, respectively, where $C_{j}=\left[C_{L}, C_{m}\right]$ representing lift and pitch moment coefficients (or any other aerodynamic coefficient). The unsteady lift and pitch moment are then calculated by adding
Duhamel's integrals with respect to $\alpha$ and $q$, i.e.

$C_{j}(t)=C_{j_{0}}+\alpha(0) C_{j \alpha}(t)+\int_{0}^{t} C_{j \alpha}(t-\tau) \dot{\alpha}(\tau) d \tau+\int_{0}^{t} C_{j q}(t-\tau) \dot{q}(\tau) d \tau$

where $C_{j_{0}}$ denote the zero-angle-of-attack lift and pitch moment coefficients and are found from static calculations; $\alpha(0)$ is the angle of attack at time zero or the initial time of motion. Note that $\alpha(0) C_{j \alpha}(t)$ is different from $C_{j_{0}}$. The motions considered here start from a steady-state solution with $q(0)=0$ and therefore the term of $q(0) C_{j q}(t)$ was not added to the equation. Using the "differential theorem of the convolution integral" [110], Eq. (22) changes to

$C_{j}(t)=C_{j 0}+\frac{d}{d t}\left[\int_{0}^{t} C_{j \alpha}(t-\tau) \alpha(\tau) d \tau\right]+\frac{d}{d t}\left[\int_{0}^{t} C_{j q}(t-\tau) q(\tau) d \tau\right]$

This equation predicts lift and pitch moment responses in the linear regime of a flow. A nonlinear model is also considered where the responses in the angle of attack depend on both the angle of attack and Mach number. Also, it is assumed that the response functions with respect to the pitch rate change with changes in freestream Mach number but do not vary with the changes in angle of attack at low to moderate angles of attack. This is a reasonable assumption based on aerodynamic prediction methods described in aircraft design textbooks [111]. The unsteady nonlinear lift and pitch moment coefficients at time $t$ are then obtained using nonlinear indicial response theory as

$$
\begin{aligned}
C_{j}(t) & =C_{j 0}(M)+\frac{d}{d t}\left[\int_{0}^{t} C_{j \alpha}(t-\tau, \alpha, M) \alpha(\tau) d \tau\right] \\
& +\frac{d}{d t}\left[\int_{0}^{t} C_{j q}(t-\tau, M) q(\tau) d \tau d \tau\right]
\end{aligned}
$$

where $M$ denotes the freestream Mach number. The response functions due to pitch rate, i.e. $C_{j q}(t, M)$ for $j=L, m$, can be estimated by using a time-dependent interpolation scheme from the observed responses. The pitch rate indicial functions are next used to estimate the second integrals in Eqs. (23) and (24), however, the estimation of nonlinear $C_{j \alpha}(t, \alpha, M)$ for $j=L, m$ needs more explanation. Assume a set of angle of attack samples of $\alpha=\left[\alpha_{1}, \alpha_{2}, \ldots, \alpha_{n}\right]$ at freestream Mach numbers of $M=\left[M_{1}, M_{2}, \ldots, M_{m}\right]$, where the spacing can be uniform or non-uniform. The indicial functions at each angle of $\alpha_{i}, i=1,2, \ldots, n$, degrees and Mach number of $M_{k}, k=1,2, \ldots, m$, are calculated by holding the angle of attack fixed at $\alpha=\alpha_{i}$ degrees, and then performing a small step in the angle of attack to $\alpha=\alpha_{i}+\Delta \alpha$. The indicial functions are then computed by taking the differences between time-varying responses occurring after the step and the steady-state solution at $\alpha=\alpha_{i}$ degrees, and dividing them by the magnitude of the step.

Ghoreyshi and Cummings [112] extended the indicial response method to include lateral aerodynamic coefficients as well. Assuming that the lateral loads only depend on side-slip angle $(\beta)$, normalized roll rate $(p)$, and normalized yaw rate $(r)$, the unsteady lateral forces and moments using indicial functions are written as

$$
\begin{aligned}
C_{j}(t) & =\frac{d}{d t}\left[\int_{0}^{t} C_{j \beta}(t-\tau, \alpha, M) \beta(\tau) d \tau\right]+\frac{d}{d t}\left[\int_{0}^{t} C_{j p}(t-\tau, M) p(\tau) d \tau\right] \\
& +\frac{d}{d t}\left[\int_{0}^{t} C_{j r}(t-\tau, M) r(\tau) d \tau\right]
\end{aligned}
$$

where $j=Y, l, n$ denote the side-force, roll and yaw moments, respectively. The functions of $C_{L \alpha}(t, \alpha, M), C_{m \alpha}(t, \alpha, M), C_{L q}(t, M)$, $C_{m q}(t, M), C_{Y \beta}(t, \alpha, M), C_{l \beta}(t, \alpha, M), C_{n \beta}(t, \alpha, M), C_{Y p}(t, M), C_{l p}(t, M)$, $C_{n p}(t, M), C_{Y r}(t, M), C_{l r}(t, M)$, and $C_{n r}(t, M)$ are unknown and the methods for calculating them in CFD will be reviewed. Note that the integral operation in the indicial theory allows for predicting airload responses from time-histories of motion. However, similar 
to the Volterra series, the indicial theory is typically accurate for weak nonlinearities.

\subsection{Surrogate-based recurrence-framework}

The set of nonlinear equations describing the CFD system can be viewed as a multi-input/multi-output dynamic system with a mathematical model in state space given by an ordinary differential equation of the form

$\dot{u}_{t}=f\left(u_{t}, x_{t}\right)$

and an output equation as

$y_{t}=h\left(u_{t}\right)$

with given initial condition $u=u_{0}$ for $t=t_{0}$ and

$u_{t} \in \mathbb{R}^{r}, \quad y_{t} \in \mathbb{R}^{n}, \quad x_{t} \in \mathbb{R}^{r}$

where $u$ is a $r$-dimensional vector of internal state variables over the field $\mathbb{R}$ of real numbers, $x$ is a vector of the inputs to system, and $y$ is $m$-dimensional vector of system outputs. For a discretetime dynamical system, the equations change to

$u_{k+1}=f\left(u_{k}, x_{k}\right)$

$y_{k}=h\left(u_{k}\right)$ for $k=0,1 \ldots$

where $k$ is an integer value showing discrete time values. The state function $f$ is a smooth function that maps the current state $u_{k}$ and the input $x_{k}$ into a new state $u_{k+1}$, and the output function $h$ maps the state $u_{k}$ into the output $y_{k}$ given as [113]

$f: \mathbb{R}^{r} \times \mathbb{R}^{n} \mapsto \mathbb{R}^{r}, \quad h: \mathbb{R}^{r} \mapsto \mathbb{R}^{m}$

In this system, the outputs can be determined from the states at time instant $k$ only, therefore the past history of the system is irrelevant [114]. If the state variables are directly measured, the functions $f$ and $h$ can be approximated using neural networks or surrogate-based models. However, in many practical situations, measuring all state variables is limited.

Referring to unsteady aerodynamic problem during pitching and plunging motions, the discretized governing equations of fluid dynamics serve as the state space functions and the input vector of $\mathbf{x}$ takes the form of

$\mathbf{x}(t)=(\alpha(t), \dot{\alpha}(t), \ddot{\alpha}(t))$

also, the output vector is defined as $y=C_{L, D, m}(t)$. In this system, the internal state vector includes $(\rho, p, u, v, w, E)$ that correspond to the values of density, pressure, velocity components, and energy at each grid point. This large amount of data makes the identification of Eq. (28) a very complex task. Fortunately, there are methods available that allow us to reconstruct the state space model by mapping only the input and output data.

For a finite-time interval and a system described by Eq. (28), Levin and Narendra [115] used the Implicit Function theorem [116] to write the output vector at any instant as a function of the past $n$ values of the inputs and the past $m$ values of the outputs, i.e.,

$y_{k+1}=\Phi\left(y_{k}, y_{k-1}, \ldots, y_{k-m+1}, x_{k}, x_{k-1}, \ldots, x_{k-n+1}\right)$

In Eq. (30), $\Phi$ is a vector-valued nonlinear function that maps the inputs to the outputs, and $m$ and $n$ are integer numbers representing the past values in the output and input, respectively. Eq. (30) preserves the characteristics of the state-space model but no longer depends on system internal states. The input-output mapping, $\Phi$, can be learned using neural networks when the time histories of input and output are available. This network is often named a recurrent neural network [114], where the network output becomes part of the next input vector [72].

Ghoreyshi et al. [36] extended the mapping function in Eq. (30) to allow for primary and secondary measurements. Assume that secondary measurements of $\tilde{y}_{t} \in \mathbb{R}^{m}$ are also available. These data are assumed to be cheaper to measure than primary data and hence they are available at many combinations of the input vector, $\tilde{x}_{t}$. An additional mapping is then defined between this system input and the secondary data, i.e.

$\tilde{y}_{k+1}=\tilde{\Phi}_{s}\left(\tilde{y}_{k}, \tilde{y}_{k-1}, \ldots, \tilde{y}_{k-m+1}, \tilde{x}_{k}, \tilde{x}_{k-1}, \ldots, \tilde{x}_{k-n+1}\right)$

The mapping in Eq. (31) is then used to predict secondary output values at the $n$ past values of the primary input vector, $x$. The function in Eq. (30) is then augmented by this evaluation in the form of

$$
\begin{aligned}
y_{k+1}= & \Phi_{t}\left(y_{k}, y_{k-1}, \ldots, y_{k-m+1}, x_{k}, x_{k-1}, \ldots, x_{k-n}\right. \\
& \left.+1, \tilde{y}_{k}, \tilde{y}_{k-1}, \ldots, \tilde{y}_{k-n+1}\right)
\end{aligned}
$$

such a model brings the information of secondary data into the mapping function and aids in reducing the computational cost. Central to the generation of the reduced-order model is the computation of the function $\Phi$ in Eqs. (30)-(32). Without a closed-form analytical expression, a numerical approximation of $\Phi$ is constructed using a number of CFD solutions. For a pitching aircraft, any motion can be expressed as a functional of three parameters, e.g., $\alpha_{0}, \alpha_{A}$, and $k$, that represent the mean angle of attack, amplitude, and reduced frequency, respectively. These independent variables form a parameter space, which represents the envelope of all possible flow conditions in which the aircraft configuration is expected to operate. To generate a consistent set of unsteady aerodynamic loads in response to a given aircraft motion time history, the training cases at which CFD solutions are calculated should be representative of the expected flow conditions. Several Design of Experiment methods are available in the literature including the Kriging-based framework [117]. Let $N_{T}$ be the number of training cases at which CFD solutions are available. Each training case consists of different combinations of the independent parameters,

$\mathbf{x}_{i}=\left(\alpha_{i}(t), \dot{\alpha}_{i}(t), \ddot{\alpha}_{i}(t), \ldots\right)$ for $i=1, \ldots, N_{T}$

and the corresponding aerodynamic loads are indicated by $y_{i}(t)$. The approximation of the function $\Phi$ is obtained by interpolating the sampled data in the form of an input/output relationship. Kriging interpolation is a common interpolation method, but for increasing number of independent parameters the problem can be ill-conditioned. An alternative approach is the multi-linear interpolation technique, which is in general faster than the Kriging interpolation.

\subsection{Radial basis functions}

The RBF model used is similar to SBRF because they are both created by the input-output mapping function of $\Phi$ given in Eq. (30), except that the function $\Phi$ is now approximated through RBF neural networks from a set of training data. The RBF network provides an approximation of the functions based on the location of data points, and is generally much faster than multi-layer feedforward neural networks [114]. Given an input vector of $\left\{X_{j}^{c}: j=1, \ldots, p\right\}, X_{j}^{c} \in \mathbf{R}$ and a corresponding output vector of $\left\{Y_{j}^{c}: j=1, \ldots, p\right\}, Y_{j}^{c} \in \mathbf{R}$, the RBF approximates the output at a new given point as

$\hat{Y}\left(X^{*}\right)=\sum_{k=1}^{P} \alpha_{k} \Phi_{i}(X)$

such that

$\hat{Y}\left(X_{j}^{c}\right)=Y_{j}^{c} \quad$ for $\mathrm{j}=1,2, \ldots, p$

where $\alpha_{k}$ are the weights of the linear combiners. The functions $\Phi_{i}$ are named Radial Basis Functions and are often described by a 
Gaussian basis function as

$\Phi_{i}(X)=\exp \left(-\frac{\left\|X^{*}-X_{j}^{c}\right\|^{2}}{\beta^{2}}\right)$

where $\beta$ is a real variable to be chosen by the user, and $\|$. denotes the Euclidean norm such that the functions $\Phi_{i}$ will vanish at sufficiently large values of $\left\|X^{*}-X_{j}^{c}\right\|$. In terms of the network structure, the RBFNN is a two-layer processing structure with one hidden layer that approximates $\Phi_{i}$ at each node. Then, the output layer is a set of linear combiners of approximation from hidden layer nodes. The network is then trained to minimize the error between the target (desired) values and the network predicted values.

\section{Review of system identification methods}

\subsection{System identification of CFD simulations}

System identification (SID) refers to the process of constructing a mathematical model to describe the relationship between input and output data of a system under testing [52]. The mathematical model structure can take various forms depending upon the intended use, i.e. what is assumed to be known, and what is to be computed. Usually wind tunnel and flight-test data are used to obtain accurate and comprehensive mathematical models of aircraft aerodynamics for aircraft flight simulation, control system design and evaluation, and dynamic analysis [118,56]. Recently, aircraft system identification has been used in cooperative approaches with CFD to take advantage of the strength of both approaches or having one approach fill in the gaps where the other cannot be used $[52,53]$. The wide range of SID tools that have been developed for aircraft system identification (including modeling techniques proposed by Morelli [56]) can easily be used to analyze CFD data computed for aircraft in prescribed motions. The modeling effort is potentially global because the independent variables $(\alpha, \dot{\alpha}, \beta$, etc.) are varied over a large range. These globally valid models and their associated smooth gradients are useful for optimization, robust nonlinear control design and global nonlinear stability, and control analysis.

\subsection{Volterra kernels}

The Volterra kernels need to be determined for creation of a Volterra-based ROM. Several methods exist to identify these kernels in the time and frequency domains. The techniques based on a continuous time impulse are proposed by George [119] and Schetzen [120] to estimate these functions. Silva [31] also used a method based on the impulse functions to directly calculate firstand second-order kernels using CFD. In his approach the firstorder kernel is a combination of the response to unit and double unit impulses at time $t_{1}=T$. The second-order kernel is a combination of two successive unit impulses at time $t_{1}=T$ and $t_{2}=T+\Delta t$ and two unit pulses, one at time $T$, and a second at time $T+\Delta t$. Although this approach is straightforward, it faces some challenges as well. The main challenge is that an impulse response is a mathematical concept and has no exact equivalent in experimental or numerical studies [121]. The numerical simulation of an impulse is very complicated as the impulse occurs over a very short period of time (theoretically for $\Delta t \rightarrow 0$ ). A computational solution is to use a discrete time impulse in CFD. However, the functions found using these simulations depend on the time discretization used and are not directly related to Volterra kernels. Milanese and Marzocca [121] used a distributed impulse instead, however the simulation of these impulse functions strongly depends on the time step used. Raveh [20] showed that the estimated kernels are very sensitive to the inaccuracies in defining an impulse function in CFD. Also, a highly nonlinear system needs higher-order kernels and the impulse approach is mainly limited to the second-order kernel identification. A way to eliminate the need for impulse simulations is the indirect identification method.

Tromp and Jenkins [122] used CFD-calculated indicial responses to identify the first-order kernel of an airfoil undergoing a pitch oscillation. For higher-order kernels, Balajewicz and Dowell [64] proposed a pseudo-inverse approach, where values of aerodynamic coefficients and the time-history of the motion variables are known from the CFD simulation and are used as a training input. The CFD solution is discrete in time, and the time step is indicated by $\Delta t$. Let $x$ denote as $x(t)=x(n \Delta t)=x[n]$. The discretetime representation of Eq. (20) can be rewritten as

$$
\begin{aligned}
y[n]= & \sum_{j=1}^{m} \cdot \sum_{k=0}^{n} H_{1}^{x_{j}}[n-k] x_{j}[k] \\
& +\sum_{j_{1}=1}^{m} \sum_{j_{2}=1}^{m} \cdot \sum_{k_{1}=0}^{n} \sum_{k_{2}=0}^{n} H_{2}^{x_{j_{1}}, x_{j_{2}}}\left[n-k_{1}, n-k_{2}\right] x_{j_{1}}\left[k_{1}\right] x_{j_{2}}\left[k_{2}\right]+\cdots
\end{aligned}
$$

The identification of discrete-time Volterra kernels involves the resolution of an overdetermined system. Values of aerodynamic coefficients and the time-history of the motion variables are known from the CFD simulation and are used as a training input. Let $\mathbf{y}=(y[0], y[1], \ldots, y[n])^{T}$ denote each aerodynamic load computed using CFD, and let A contain the permutations of input parameters relevant to the unsteady motion. Eq. (37) can be recast in the form of

$\mathbf{y}=\mathbf{A b}$

where the vector b contains the unknown Volterra kernels. The matrix $\mathbf{A}$ is in general non-square, with more rows than columns. Several numerical methods are available to solve least squares problems, e.g., direct inversion of $\mathbf{A}^{T} \mathbf{A}$, Gaussian elimination, Moore-Penrose generalized inverse approach and QR factorization. However, the Moore-Penrose approach and QR factorization are more accurate than Gaussian elimination and the direct inversion solutions. The cost of $\mathrm{QR}$ factorization is $\mathcal{O}\left(n^{2}\right)$, and the Moore-Penrose inversion involves $\mathcal{O}\left(n^{3}\right)$ operations. Note that computational resources attributable to the identification of the Volterra kernels grow exponentially with order. Increasing the order of the Volterra series introduces a requirement for a training maneuver of sufficient duration. A remedy to this is the use of a simplified form of the kernel parametric structure. For example, Balajewicz et al. [105] proposed to set all off-diagonal terms of the kernel to zero, i.e.

$$
\begin{aligned}
& H_{p}^{x_{j_{1}}, x_{j_{2}}, \ldots, x_{j_{p}}}\left[n-k_{1}, n-k_{2}, \ldots, n-k_{p}\right]=0 \\
& \quad \text { for } k_{1} \neq k_{2} \neq \cdots \neq k_{p}
\end{aligned}
$$

The Volterra kernels are then identified from Eq. (38) solving for $\mathbf{b}$, with $\mathbf{y}$ and $\mathbf{A}$ being known for a training maneuver. The matrix $\mathbf{A}$ is then recomputed for a novel maneuver, and the low-order model in Eq. (38) is used to predict the resulting unsteady aerodynamic loads in place of the full-order system.

\subsection{Indicial functions}

The indicial functions can be estimated via analytical or computational methods. The experimental tests are practically nonexistent for impulse and step response functions. Wagner [87] was the first to solve the indicial lift response of a thin airfoil due to a step change in the angle of attack in incompressible flow, the so-called Wagner function. His function is a single parameter function and is sufficient to model the plunging motions of a thin airfoil in incompressible flows. 


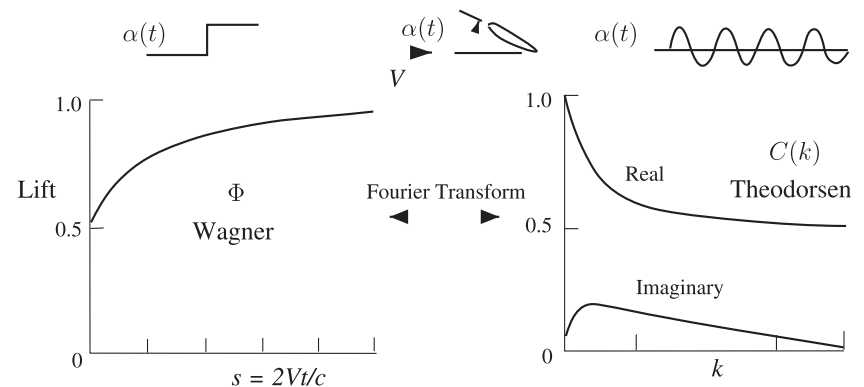

Fig. 3. Fourier transform relationship between oscillatory and indicial aerodynamics [124].

Since Wagner's original work, there have been numerous contributions to obtain the approximations of response functions in compressible flow as well; many of these solutions are reviewed by Bisplinghoff et al. [123]. The most common way of calculating the indicial functions in compressible flow is by using harmonic (oscillatory) motions [76]. The Wagner function ( $\Phi$ in Eq. (3)) and Theodorsen function $(C(k)$ in Eq. (9)) are related through a Fourier integral or Laplace transform as shown in Fig. 3, because both solutions are based on the same flow equations assuming the same boundary conditions [124,125]. Similar relations can be developed for the compressible indicial responses [76]. Mazelsky and Drischler [126] used oscillatory data to obtain the indicial lift and pitch moment responses for Mach numbers of 0.5 and 0.6. A similar approach was used later by Dowell [127] to obtain the indicial functions for transonic Mach numbers as well. However, the derived indicial functions using harmonic motions depend largely on the quality of motion, e.g. amplitude and frequency.

Recently, CFD simulations have been used for the approximation of response functions. The earliest computational efforts involved the small perturbation potential code of Ballhaus [24] and the Euler code of Magnus [128], both for applications to airfoils. Singh and Baeder [129] used a surface transpiration approach to directly calculate the angle of attack indicial response using CFD. However, this approach has not been tested for pitch rate indicial functions and aircraft configurations. Also, the approach is not available in many commercial flow solvers that do not provide access to the source code. Recently, Ghoreyshi et al. [27] described an approach based on a grid motion technique for CFD-type calculation of linear and nonlinear indicial functions. This approach will be briefly described next.

Assuming that the flow solver allows all translational and rotational degrees of freedom, the location of a reference point on the aircraft is specified at each time step. In addition the rotation of the aircraft about this reference point is defined using the rotation angles of yaw, pitch, and roll. The aircraft reference point velocity, $v_{a}$, in an inertial frame is calculated to achieve the required angles of attack and sideslip and the forward speed. The velocity is then used to calculate the aircraft location at each time step. The initial aircraft velocity, $v_{0}$, is specified in terms of Mach number, angle of attack, and side-slip angle. The instantaneous aircraft location for the motion is then defined from the relative velocity vector, $v_{a}-v_{0}$.

For CFD-type calculation of a step change in angle of attack, the grid immediately starts to move at $t=0$ to the right and downward as shown in Fig. 4. The translation continues over time with a constant velocity vector. Since there is no rotation, all the effects in aerodynamic loads are from changes in the angle of attack. For a unit step change in pitch rate, the grid moves and rotates simultaneously. The grid starts to rotate with a unit pitch rate at $t=0$. To hold the angle of attack zero during the rotation, the grid moves right and upward as shown in Fig. 4.

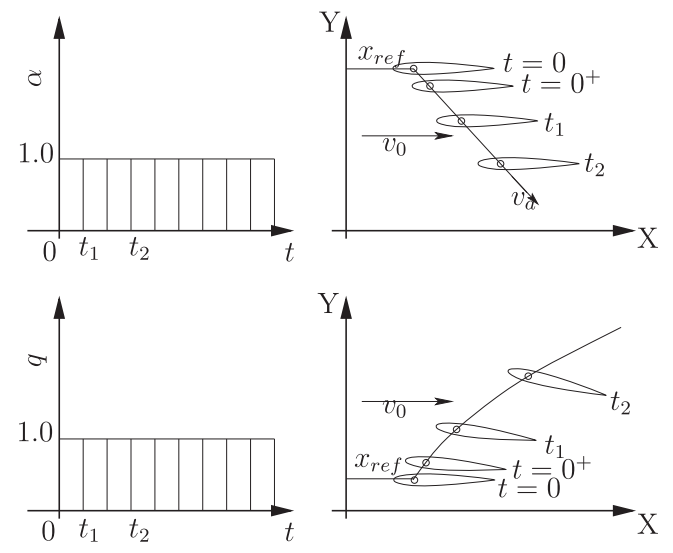

Fig. 4. The grid motion for modeling a step change in angle of attack and pitch rate.

Ghoreyshi and Cummings [112] later used this approach to generate longitudinal and lateral indicial functions for a generic unmanned combat air vehicle (UCAV) and used these functions for predicting the aerodynamic responses to aircraft 6DoF maneuvers. However, extending the model to predict airloads of these maneuvers could be computationally expensive because a large number of indicial functions need to be calculated for each combination of input parameters.

In more detail, the indicial method assumes the linearity of output response with respect to input variables [130]. This means the predicted indicial functions can be used for only small amplitude oscillations at a fixed Mach number similar to the one used for predicting indicial functions [130]. For motions at compressible speeds and large amplitude, many indicial functions need to be generated for each combination of angle of attack and freestream Mach number. It should be noted, however, that these models are still cheaper than full-order simulations because the ROMs based on indicial functions eliminate the need to repeat calculations for each frequency [24].

In order to reduce computational cost of model creation, a special time-dependent surrogate-based modeling approach was used by Ghoreyshi and Cummings [112] that predicts indicial responses for a new point from available (observed) responses. These observed responses were viewed as a set of time-correlated spatial processes where the output is considered a timedependent function. Assume an input vector of $\mathbf{x}(t)=\left(x_{1}(t)\right.$, $\left.x_{2}(t), \ldots, x_{n}(t)\right)$ where $n$ represents the dimensionality of the input vector. To construct a surrogate model for fitting the input-output relationship, the unsteady aerodynamic responses corresponding to a limited number of input parameters (training parameters or samples) need to be generated. Design of Experiment methods, for example, can be used to select $m$ samples from the input space. The input matrix $\mathbf{D}(m \times n)$ is then defined as

$\mathbf{D}=\left[\begin{array}{cccc}x_{11} & x_{12} & \cdots & x_{1 n} \\ x_{21} & x_{22} & \cdots & x_{2 n} \\ \vdots & \vdots & \vdots & \vdots \\ x_{m 1} & x_{m 2} & \cdots & x_{m n}\end{array}\right]$

where rows correspond to different combinations of the design parameters. For each row in the input matrix, a time-dependent response was calculated at $p$ discrete values of time, and this information is summarized in the output matrix of $\mathbf{Z}(m \times p)$ as

$\mathbf{Z}=\left[\begin{array}{cccc}y_{11} & y_{12} & \cdots & y_{1 p} \\ y_{21} & y_{22} & \cdots & y_{2 p} \\ \vdots & \vdots & \vdots & \vdots \\ y_{m 1} & y_{m 2} & \cdots & y_{m p}\end{array}\right]$ 
1) Model Training

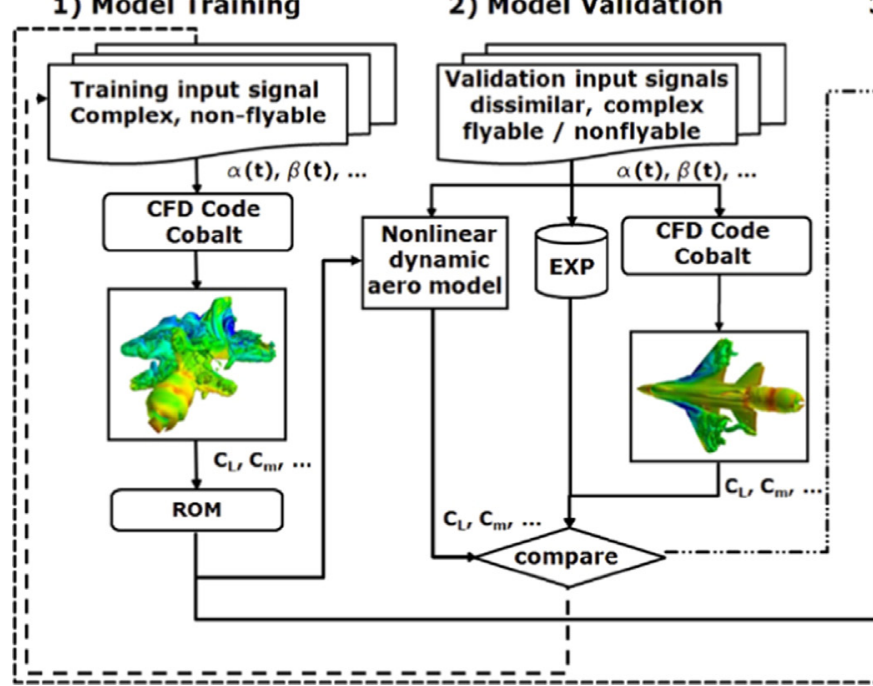

3) Model Prediction

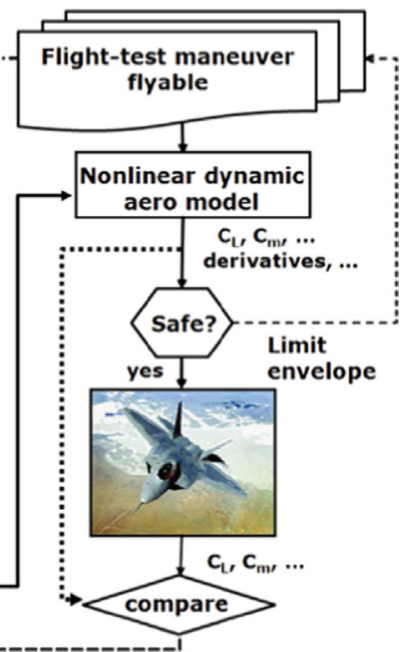

Fig. 5. Stability and control aerodynamic loads model-development process [19].

where for aerodynamic loads modeling, $p$ equals the number of iterations used in time-marching CFD calculations. The objective of surrogate modeling is to develop a model that allows for predicting the aerodynamic response of $\mathbf{y}\left(\mathbf{x}_{\mathbf{0}}\right)=\left(y_{01}, y_{02}, \ldots, y_{0 p}\right)$ at a new combination of input parameter of $\mathbf{x}_{\mathbf{0}}$. To construct this surrogate model, the responses at each time step are assumed as a separate set, such that each column of the output matrix is a partial realization of the total response. In this sense, $p$ surrogate models are created; they are denoted as $\mathbf{Z}_{\mathbf{i}}(\mathbf{D})$ for $i=1,2, \ldots, p$. A universaltype Kriging function [131] is then used to approximate these models. For more details about creating Kriging models, the reader is referred to Ghoreyshi et al. [132]. Having created Kriging models for each $\mathbf{Z}_{\mathbf{i}}(\mathbf{D})$ function, the total response at $\mathbf{x}_{\mathbf{0}}$ is then combination of predicted values of each model, i.e.

$\tilde{\mathbf{Z}}\left(\mathbf{x}_{\mathbf{0}}\right)=\left(\tilde{\mathbf{Z}}_{\mathbf{1}}\left(\mathbf{x}_{\mathbf{0}}\right), \tilde{\mathbf{Z}}_{\mathbf{2}}\left(\mathbf{x}_{\mathbf{0}}\right), \ldots, \tilde{\mathbf{Z}}_{\mathbf{p}}\left(\mathbf{x}_{\mathbf{0}}\right)\right)$

where shows that Kriging model is an approximation of the actual function. For modeling aerodynamics of a maneuvering aircraft, the input matrix of $\mathbf{D}$ includes combinations of angle of attack and Mach number, i.e.

$\mathbf{D}=\left[\begin{array}{cc}\alpha_{1} & M_{1} \\ \alpha_{2} & M_{2} \\ \vdots & \vdots \\ \alpha_{m} & M_{m}\end{array}\right]$

so in this case $n=2$ and $\mathbf{x}_{\mathbf{0}}=\left[\alpha_{0}, M_{0}\right]$. The output vector is also defined as $\mathbf{y}=\left[C_{L}, C_{m}, C_{Y}, C_{l}, C_{n}\right]$ and therefore the output matrices of $\mathbf{Z}_{\mathbf{j}}$ are

$\mathbf{Z}_{\mathbf{j}}=\left[\begin{array}{cccc}C_{j_{11}} & C_{j_{12}} & \cdots & C_{j_{1 p}} \\ C_{j_{21}} & C_{j_{22}} & \cdots & C_{j_{2 p}} \\ \vdots & \vdots & \vdots & \vdots \\ C_{j_{m 1}} & C_{j_{m 2}} & \cdots & C_{j_{m p}}\end{array}\right]$

where $j=[L, m, Y, l, n]$ represents lift, pitch moment, side force, roll moment, and yaw moment coefficients, respectively. The unsteady effects in drag force were assumed to be small and are not included.

\subsection{Training maneuvers}

The focus of S\&C research at USAFA and AFSEO has been to effectively incorporate CFD into the model-development process
Table 1

Special training maneuvers.

\begin{tabular}{ll}
\hline Maneuver & Definition \\
\hline Linear chirp & $\alpha(t)=\alpha_{0}+\alpha_{A} \sin \left(\omega t^{2}\right)$ \\
Spiral & $\alpha(t)=\alpha_{0}+\alpha_{A} t \sin (\omega t)$ \\
Schroeder & $\alpha(t)=\alpha_{0}+\alpha_{A} \sum_{k=1}^{n} \sqrt{\frac{1}{2 N}} \cos \left(\frac{2 \pi k t}{T}-\frac{\pi k^{2}}{N}\right)$ \\
\end{tabular}

using dynamic CFD predictions of complete aircraft configurations. This has lead to an innovative approach for modeling aircraft S\&C characteristics, the details of which can be found in McDaniel et al. [19]. A schematic of the approach is graphically represented in Fig. 5, and a summary is as follows:

1. CFD simulations are performed using computational training maneuvers designed to excite the relevant flow physics encountered during actual missions.

2. A mathematical ROM is built for the aircraft response.

3. The model is validated by comparing CFD simulations against model predictions.

4. Predictions of all flight test points are made using the model to determine the expected behavior of the aircraft.

A training maneuver (or multiple maneuvers) is needed to provide enough information to learn the mapping between inputs and outputs in Eq. (30) by using SBRF and RBF methods. Previous studies to generate training maneuvers for aerodynamic characteristics [56,133-136] are limited by the range of the motion frequency content. A ROM identified from such a maneuver has limitations with respect to S\&C applications. Thus, the basic requirement for a training maneuver to generate a reliable ROM in S\&C applications is that it sufficiently covers the desired regressor space of state variables. A ROM built on data produced by such motions can then be used to predict the aircraft aerodynamic behavior within the regressor space. The systematic coverage of the regressor space can be, in general, treated as an optimization problem of filling the multidimensional space with strong constraints resulting from the fact that some axes of the regressor space do not represent an independent variable. For the current study, three special training maneuvers are reviewed: linear chirp, spiral, and Schroeder. These maneuvers are defined in Table 1 and some examples are shown in Fig. 6. 

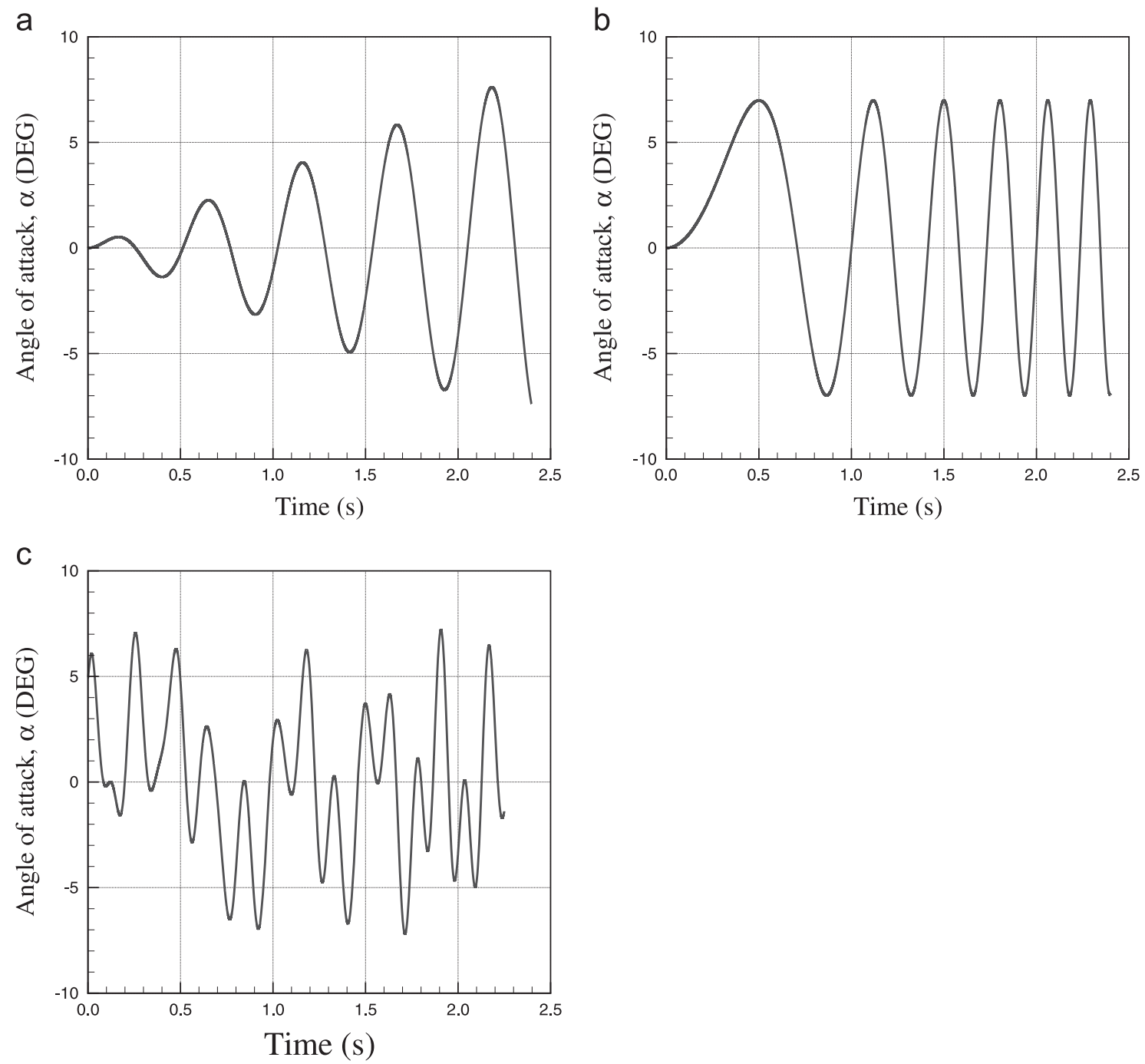

Fig. 6. A spiral, a chirp, and a Schroeder training maneuver. (a) spiral maneuver; (b) chirp maneuver; and (c) Schroeder maneuver.

In Table $1, \alpha_{0}$ is the mean angle of attack, $\alpha_{A}$ is the amplitude, and $\omega$ is the angular velocity. The chirp maneuver used has a constant amplitude and linearly increasing frequency in time. In the spiral maneuver, the amplitude increases linearly in time, as does the angle of attack. The Schroeder maneuver is a multi-stage frequency sweep consisting of multiple cosine terms with a specified phasing. This maneuver has three parameters that enable direct control of the regressor space coverage: maneuver amplitude, $\alpha_{A}$, maneuver length, $T$, and the number of frequencies in the maneuver, $N$.

\section{Flow solver}

The flow solver used for this study is the Cobalt code [137] that solves the unsteady, three-dimensional and compressible NavierStokes equations in an inertial reference frame. In Cobalt, the Navier-Stokes equations are discretized on arbitrary grid topologies using a cell-centered finite volume method. Second-order accuracy in space is achieved using the exact Riemann solver of Gottlieb and Groth [138], and least squares gradient calculations using QR factorization. To accelerate the solution of the discretized system, a point-implicit method using analytic first-order inviscid and viscous
Jacobians is used. A Newtonian sub-iteration method is used to improve the time accuracy of the point-implicit method. Tomaro et al. [139] converted the code from explicit to implicit, enabling Courant-Friedrichs-Lewy (CFL) numbers as high as $10^{6}$. Some available turbulence models are the Spalart-Allmaras (SA) model [140], Wilcox's $k-\omega$ model [141], and Mentor's SST model [142].

\section{Validation test cases}

A range of test cases, from airfoil to full aircraft, have been used to evaluate and validate the reduced order methods. Note that the purpose of this review is not to present validation results of CFD codes or grid-independence study, but rather to validate ROM predictions against CFD data. Therefore, only one CFD code and only one mesh (fine size) was used for each test case based on the previous experience with CFD and mesh generation tools in predicting unsteady aerodynamics.

All RANS meshes were generated in two steps. In the first step, the inviscid tetrahedral mesh was generated using the ICEMCFD code. This mesh was then used as a background mesh by TRITET $[143,144]$ which builds prism layers using an advancing front technique. TRITET rebuilds the inviscid mesh while respecting the 
a

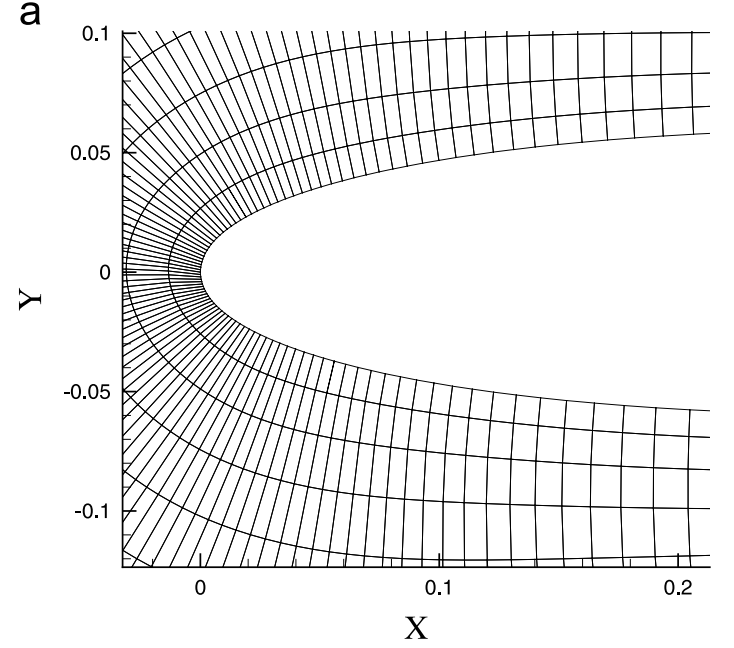

C

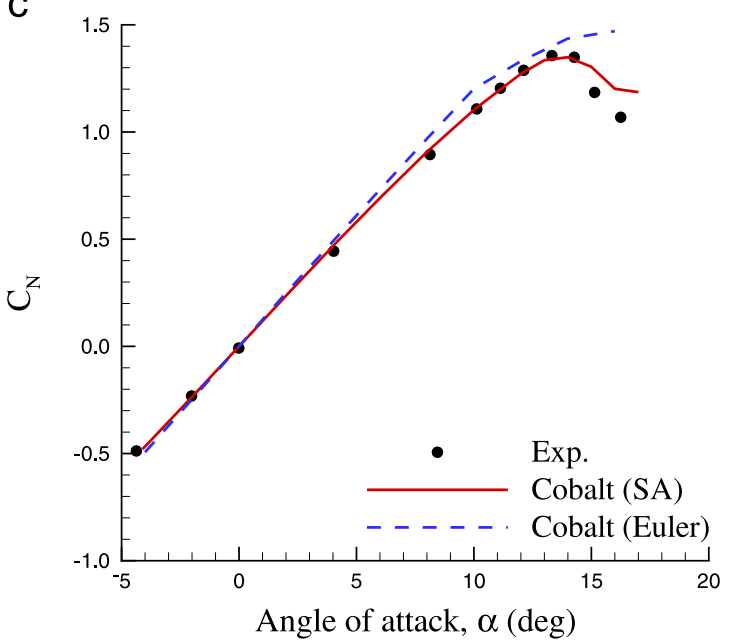

e

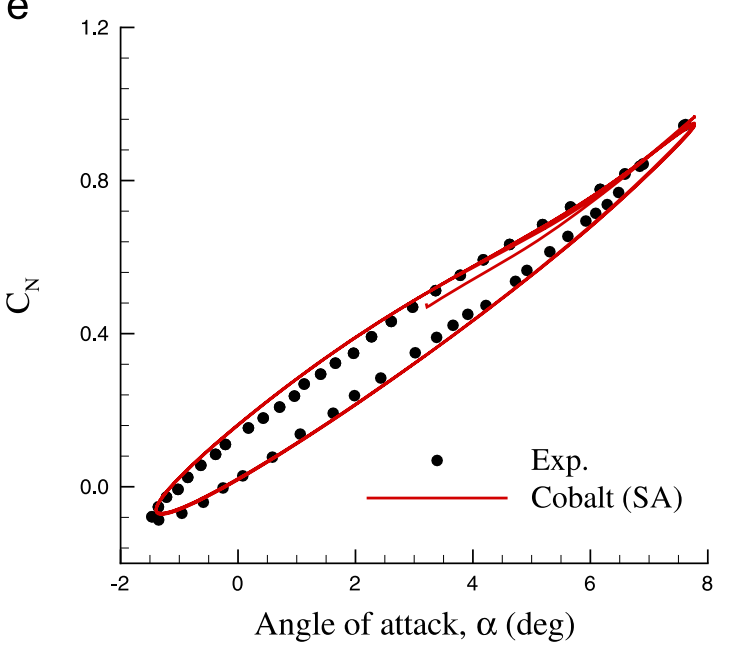

b

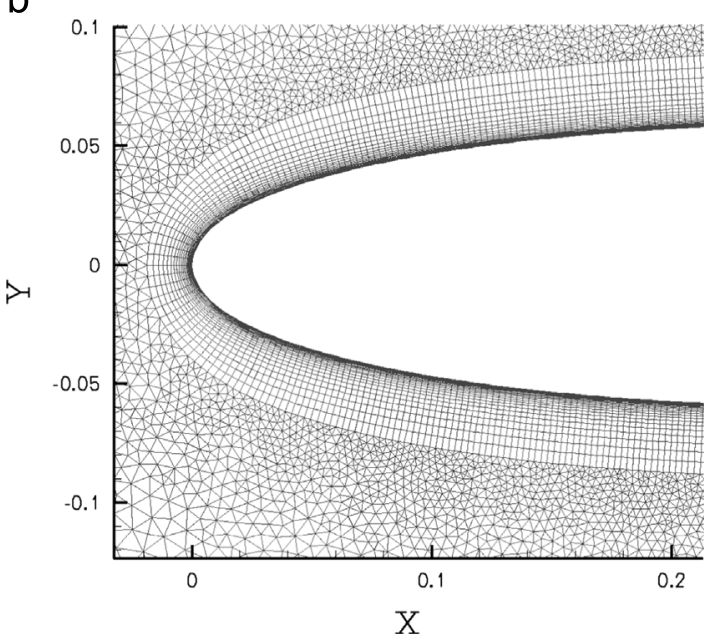

d

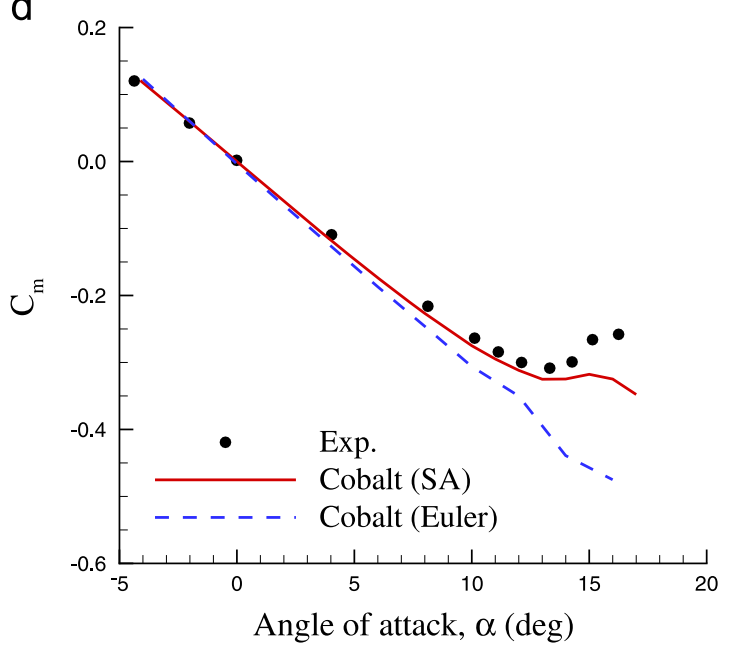

f

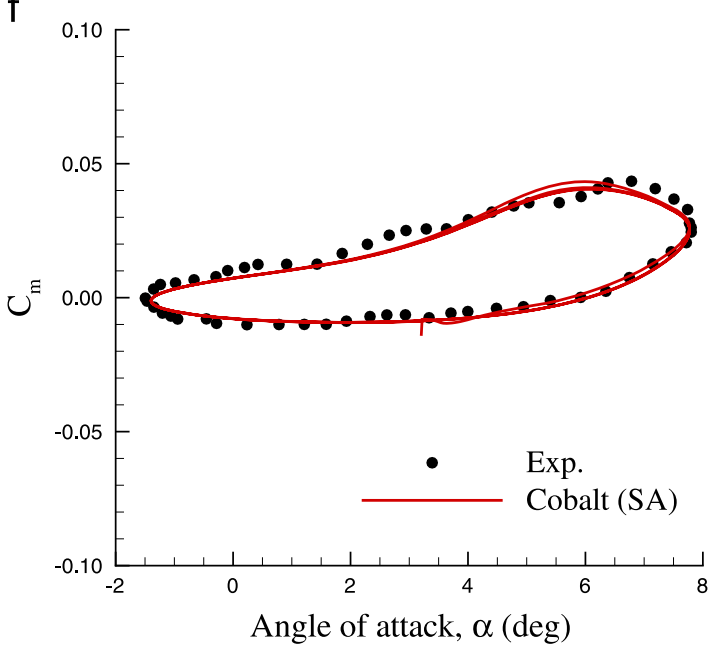

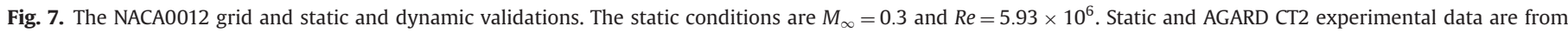

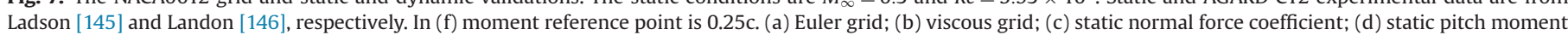
coefficient; (e) normal force coefficient of AGARD CT2; and (f) pitch moment coefficient of AGARD CT2.

size of the original inviscid mesh from ICEMCFD. Simulations of aircraft configurations were run on the Cray XE6 machines at the Engineering Research Development Center (ERDC) (Garnet with $2.7 \mathrm{GHz}$ core speed and Chugach with $2.3 \mathrm{GHz}$ core speed) which have approximately 20,000 and 11,000 cores, respectively.

\subsection{NACA 0012 Airfoil}

Both Euler and RANS meshes are available as shown in Fig. 7. In both meshes, the minimal distance from the body to each of the outer boundaries is $20 c$, where $c$ is the airfoil chord. The Euler 
mesh was generated using Gridgen version 15.0, and is a structured mesh generated by normal extrusion of surface connectors. The overview of the Euler mesh is shown in Fig. 7(a). The RANS mesh volume is rectangular with the airfoil geometry centrally located. The no-slip adiabatic wall boundary conditions are employed at the body surface, and a modified Riemann-invariant condition is implemented at the far-field boundary. The RANS mesh has prisms cells near the airfoil and tetrahedra cells elsewhere. The pitch axis is set to $0.25 c$, but the moment reference point is at the leading edge unless stated otherwise. The overview of the RANS mesh is shown in Fig. 7(b).

The static Euler and RANS calculations are shown in Fig. 7. Note that Euler calculations are significantly faster than RANS calculations (on the order of five times faster for the meshes used in this work). The simulations correspond to $M=0.3$ and $R e=8.93 \times 10^{6}$ in order to match experimental data from Ladson [145]. All RANS simulations were performed using the SA turbulence model. Euler and RANS predictions compare well with the experiments at low angles of attack as shown in Fig. 7. The RANS model accurately predicts the maximum lift, but the stall region predictions do not match as well. The Euler model predicts the slopes of lift and pitch moment fairly well up to moderate angles of attack. Differences between the Euler predictions and experiments are observed at high angles of attack due to the inviscid assumption, although the Euler simulations show flow separation on the upper surface at higher angles of attack resulting in a fall in lift slope. Cobalt in Euler mode predicts the secondary boundary layer separation at high angles of attack due to adverse pressure gradients, however, these predictions are not accurate.

A dynamic test case (AGARD CT2) was also selected with available experimental data from Landon [146]. The AGARD CT2 is a pitch forced oscillation with test conditions summarized in Table 2. The CFD simulation of the RANS grid ran for three cycles with a non-dimensional time-step of $t^{*}=V t / c=0.01$. The results are shown and compared with experimental data in Fig. 7. Even for this unsteady case, very good agreement was found, although

Table 2

Description of the AGARD CT2 test conditions.

\begin{tabular}{ll}
\hline Test conditions & Values \\
\hline Mach number, $M$ & 0.6 \\
Mean incidence, $\alpha_{0}$ & $3.16^{\circ}$ \\
Pitch amplitude, $\alpha_{A}$ & $4.59^{\circ}$ \\
Reduced frequency, $k$ & 0.0811 \\
Reynolds number, $R e$ & $4.8 \times 10^{6}$ \\
\hline
\end{tabular}

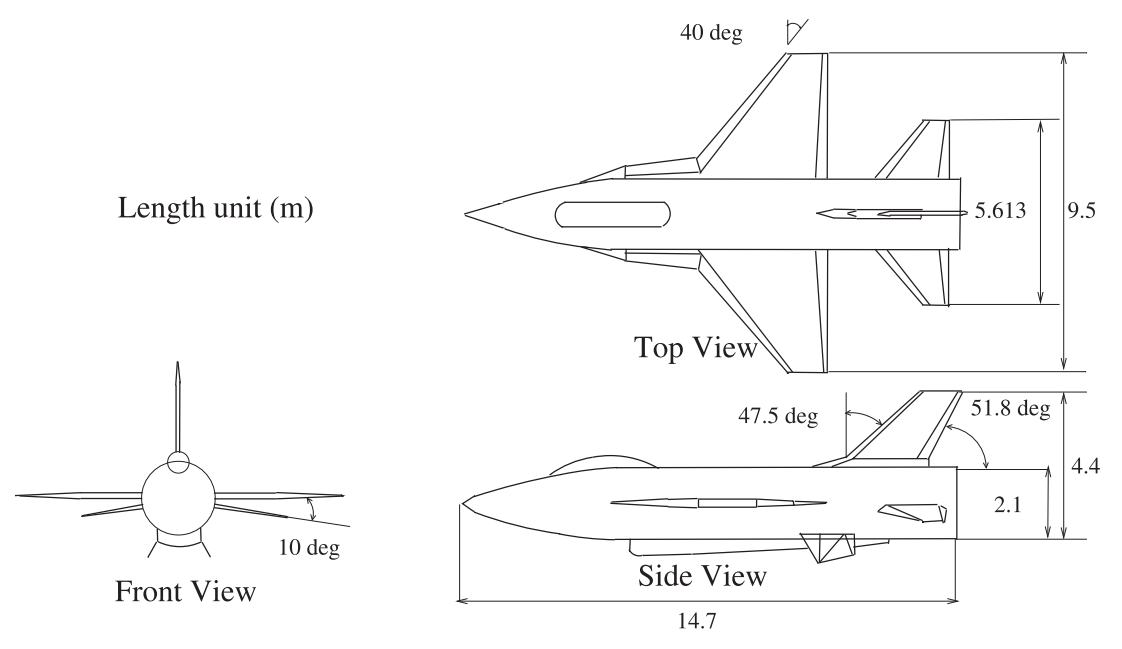

Fig. 8. Standard dynamic model (SDM) layout [150]. the angles of attack all fall within the linear range. These predictions gave confidence in the ability of the current numerical approach to predict unsteady aerodynamics.

\section{2. $S D M$}

The Standard Dynamics Model (SDM) is a generic fighter configuration based on the F-16 planform. The model includes a slender strake-delta wing, horizontal and vertical stabilizers, ventral fins, and a blocked off inlet. The three-view drawing is shown in Fig. 8. This geometry has been tested extensively at various wind tunnel facilities to collect aerodynamic data [147-149]. Note that slightly different geometries were used in these previous studies.

A full-span geometry mesh is available as shown in Fig. 9. The mesh has around 9 million points and 19.5 million cells. The wind tunnel experiments [150] were used to validate the CFD predictions at low speeds. The conditions of the tests were $V=110 \mathrm{~m} / \mathrm{s}$, $R e=0.57$ million and $\beta=5^{\circ}$ for $\alpha=0-90^{\circ}$. All CFD simulations were run at free-stream conditions consistent with flow conditions in the wind tunnel tests. For flow solution, the RANS equations are discretized by second-order spatial and temporal operators. The turbulence models used are SA, SARC, and Menter's SST.

The static force and moment coefficients were compared with experimental data in Fig. 10. The comparison showed that there is a good agreement between the RANS predictions and the measurements for angles of attack below $25^{\circ}$. However, all

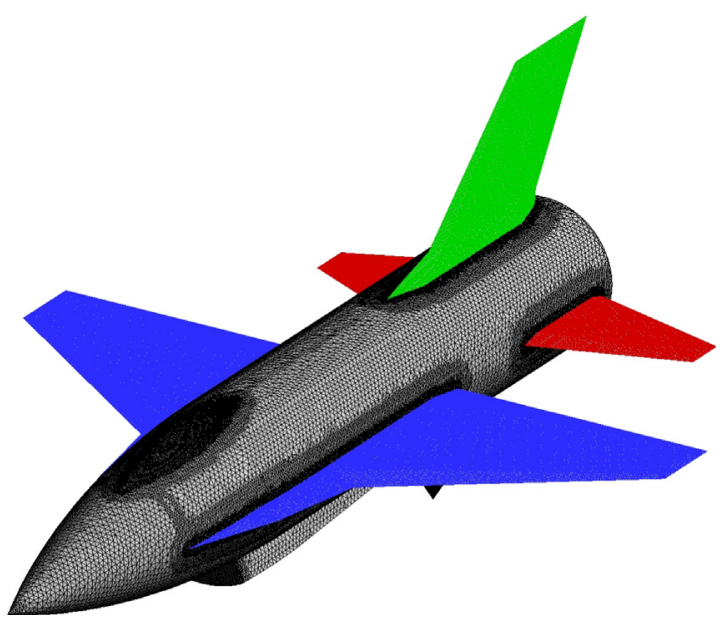

Fig. 9. The SDM aircraft surface mesh model. 

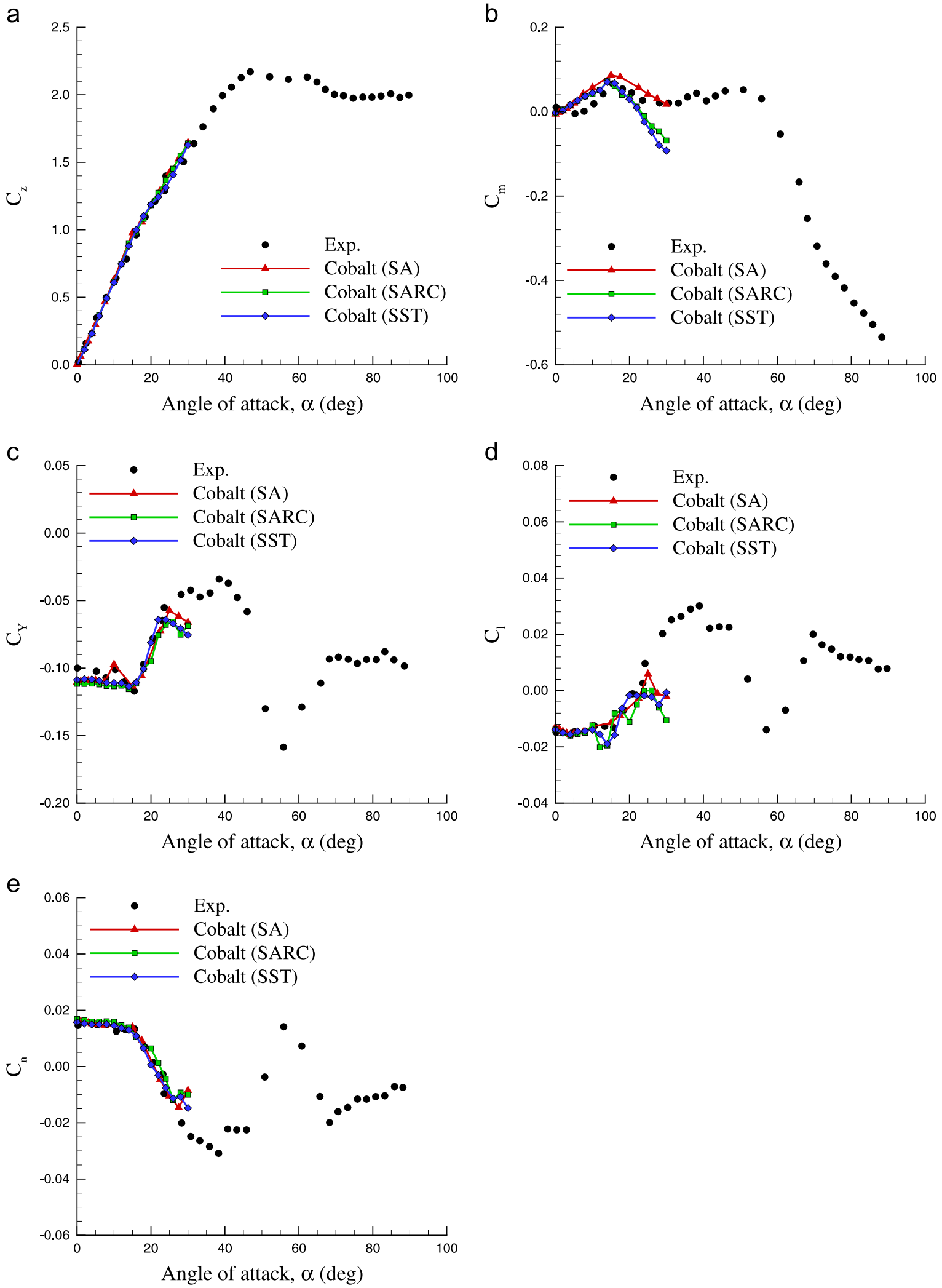

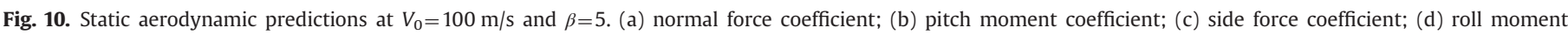
coefficient; and (e) yaw moment coefficient.

turbulence models predicted a positive pitch moment slope at zero degrees angle of attack, while experiments show a falling trend at this angle. This was likely due to different inlet geometries used in the wind tunnel models. At $5^{\circ}$, a pair of vortices emanating from the strake and wing leading-edge have formed as shown in
Fig. 10(a). These vortices do not exhibit breakdown and do not interact over the airframe. The vortex formation causes an additional increase of lift and pitch moment coefficients. In experimental tests, the sign of the pitch moment is reversed at this angle, while CFD shows a jump in the moment rate of increase. No 

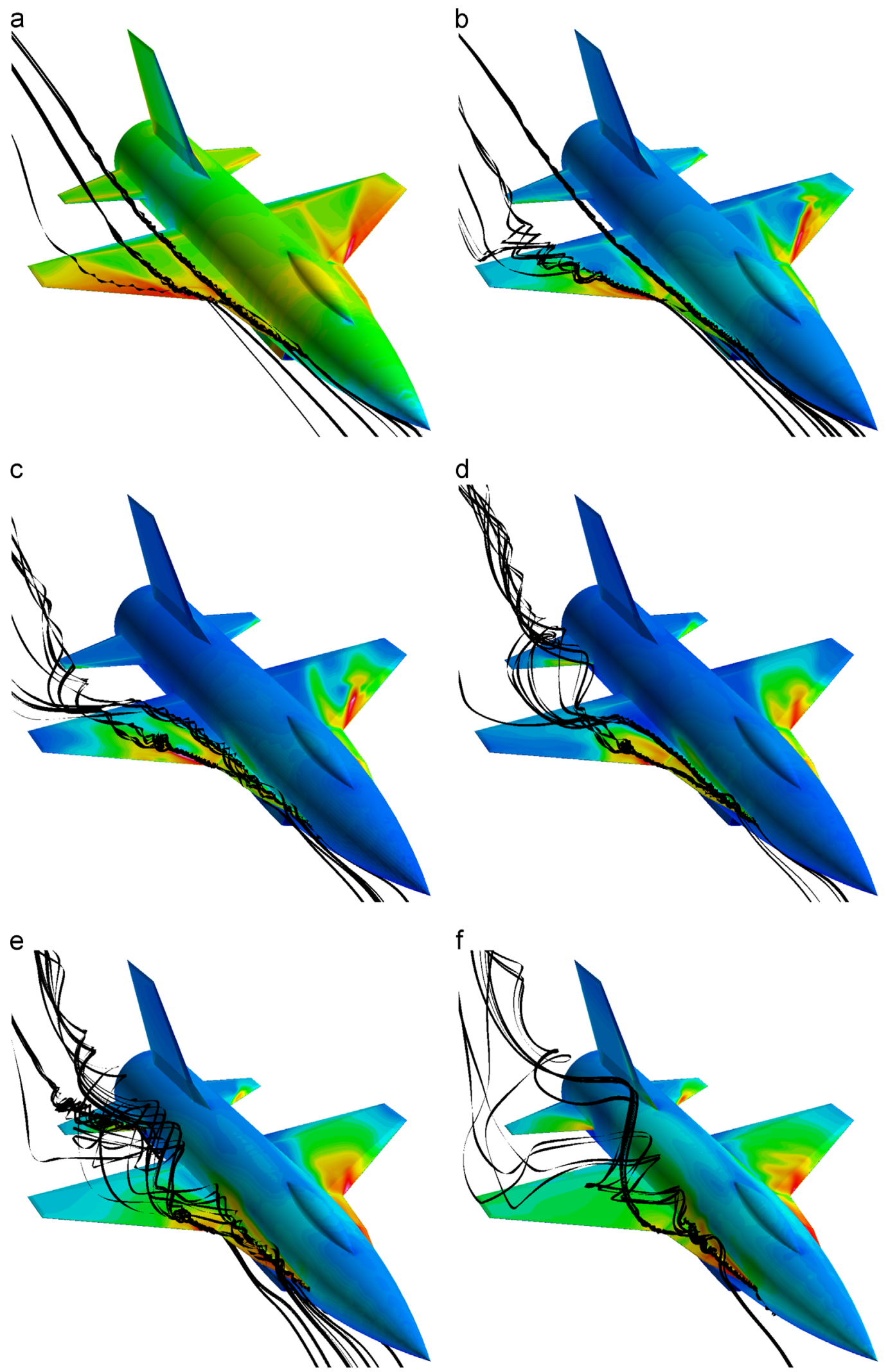

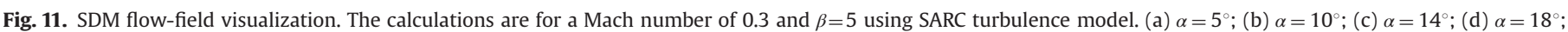
(e) $\alpha=22^{\circ}$; and (f) $\alpha=25^{\circ}$.

significant changes on lateral force and moment coefficients were observed at this angle.

The SDM vortices grow in size and strength with increasing angle of attack. At $10^{\circ}$, the center of the wing vortex is shifted laterally, while the shedding point is moved forward as shown in Fig. 11(b). There are still no signs of vortex breakdown, and the lateral moments slightly change due to the wing vortex movement. Around $14^{\circ}$, the two vortices wind around each other 
towards the trailing edge of the wing as shown in Fig. 11(c). With a slight increase in angle of attack, the wing vortex appears to breakdown quickly as shown in Fig. 11(d). The vortex breakdown leads to a smaller lift rate of increase and a negative pitch moment slope. The vortex breakdown phenomenon is asymmetric and hence the lateral force and moment coefficients suddenly start to change very rapidly. At $22^{\circ}$ the strake vortex is also burst as shown in Fig. 11(e). Finally, at $25^{\circ}$ there is no sign of a wing vortex as shown in Fig. 11(f).

\subsection{SACCON UCAV}

The SACCON geometry and experimental data were provided to the partners participating in NATO RTO Task Group AVT-161 (Assessment of Stability and Control prediction Methods for NATO

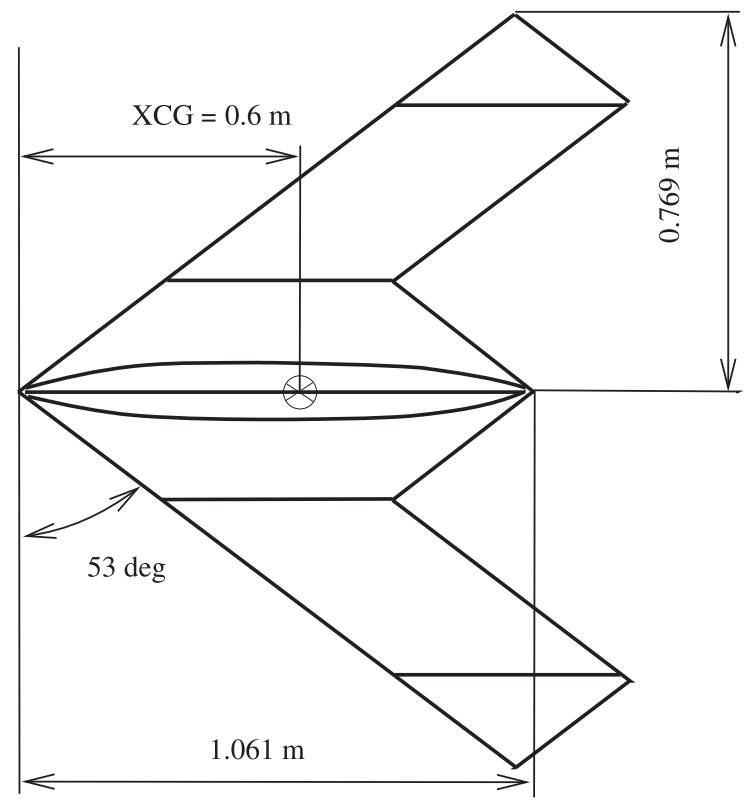

Fig. 12. The SACCON geometry [151].
Air and Sea Vehicles) [151]. The objective of this task group was to evaluate CFD codes against wind tunnel results. The vehicle planform and section profiles were defined in cooperation between the German Aerospace Center (DLR) and EADS-MAS. DLR adjusted the pre-design geometry for wind tunnel design purposes which actually led to a higher overall thickness at the root chord to provide enough space for the internal strain gauge balance. The aircraft has a lambda wing planform with a leading edge sweep angle of $53^{\circ}$ as shown in Fig. 12. The root chord is approximately $1 \mathrm{~m}$, the wing span is $1.53 \mathrm{~m}$, the reference chord is $0.48 \mathrm{~m}$, and the reference area is $0.77 \mathrm{~m}^{2}$. The main sections of the model are the fuselage, the wing section, and wing tip. The configuration is defined by three different profiles at the root section of the fuselage, two sections with the same profile at the inner wing, forming the transition from the fuselage to wing and the outer wing section. Finally, the outer wing section profile is twisted by $5^{\circ}$ around the leading edge to reduce the aerodynamic loads and shift the onset of flow separation to higher angles of attack.

The wind tunnel model was designed and manufactured at NASA Langley Research Center (LaRC). The model was designed to accommodate a belly sting mount for tests in the German-Dutch Low Speed Wing Tunnel (DNW-NWB) in Braunschweig and the $14 \mathrm{ft} \times 22 \mathrm{ft}$ low speed wind tunnel at NASA LaRC $[151,152]$. The high-angle-of-attack flow around SACCON is very complicated and unsteady due to vortical flow formation, vortex interaction, and vortex breakdown. SACCON also has complicated aerodynamic characteristics at non-symmetric flow conditions. Some experimental aerodynamic behavior of the SACCON configuration in the lateral direction are shown in Fig. 13. The experimental results [151] show that side force, yaw and roll moments are a nonlinear function of angle of attack, most significantly above an angle of attack of $10^{\circ}$. Fig. 13 also shows that the angle of attack dependency can be seen in the roll moment even at low angles of attack. The lateral coefficients are nearly linear with side slip angle for angles of attack below $15^{\circ}$, and become increasingly dependent on side-slip for angles of attack above $15^{\circ}$ as shown in Fig. 13.

Two meshes are available, the first uses a belly mounted sting present in the experiments and the second has no sting. The grid including sting geometry is shown in Fig. 14. This grid contains
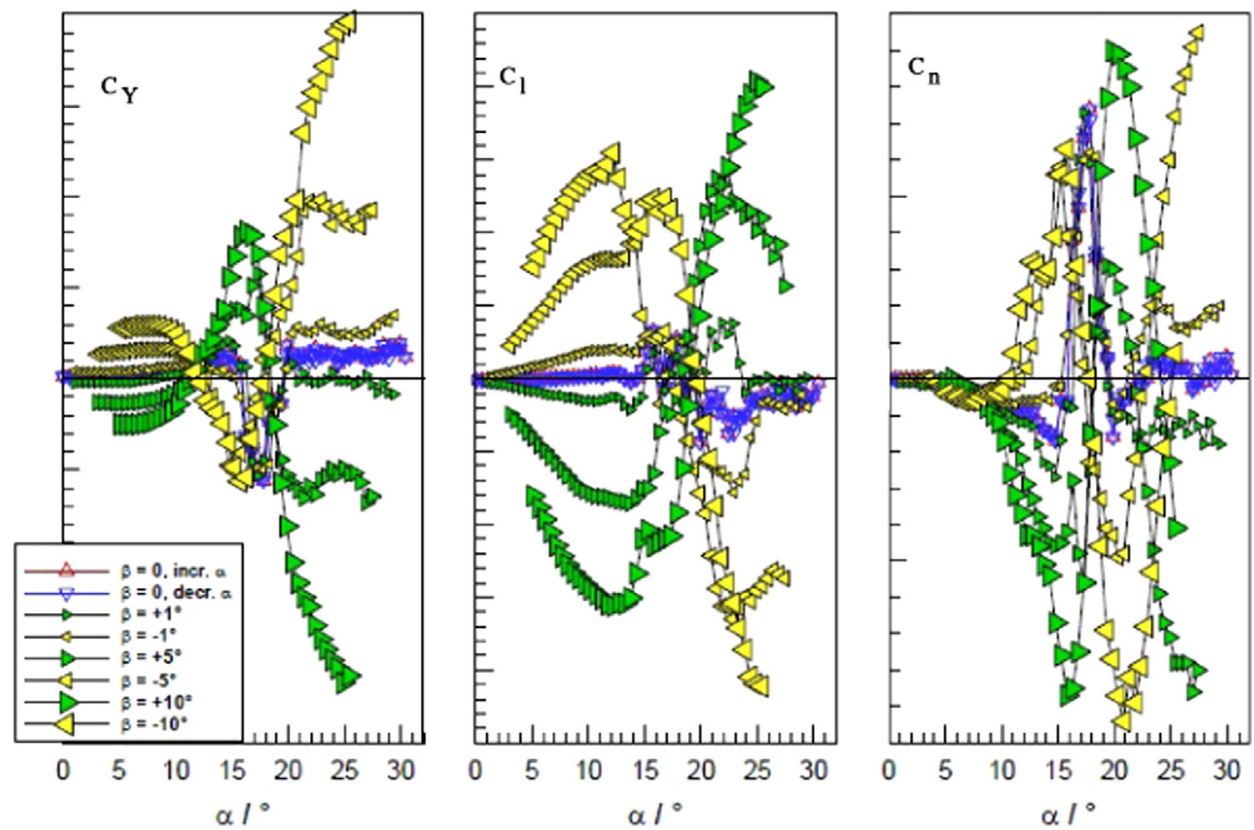

Fig. 13. SACCON side force, roll moment, yaw moment versus angle of attack for different angles of side slip [151]. 
a

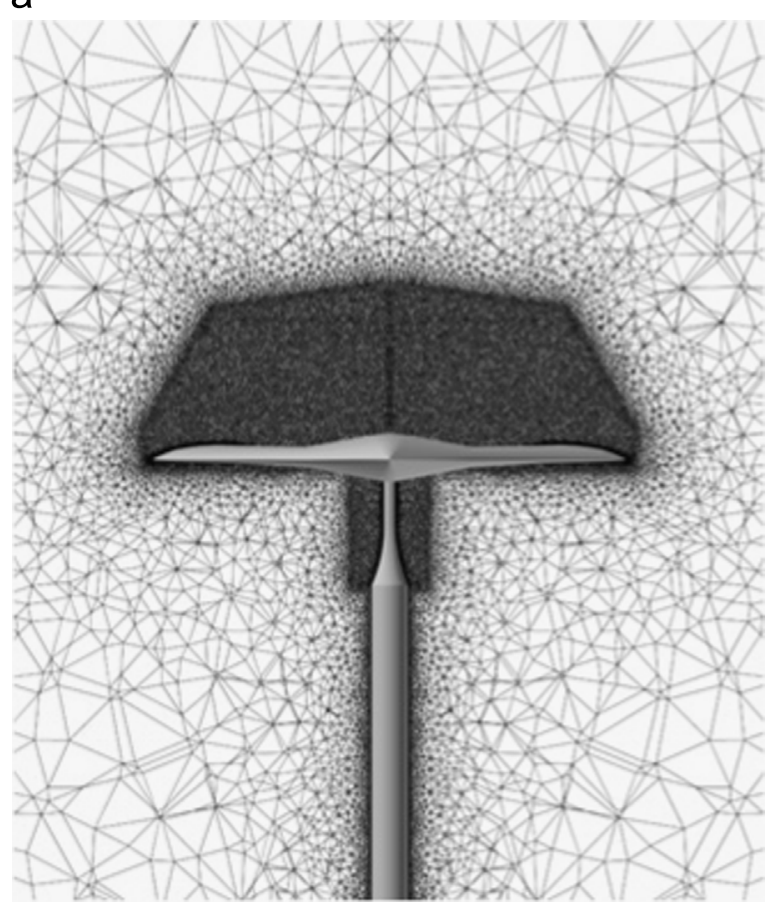

C

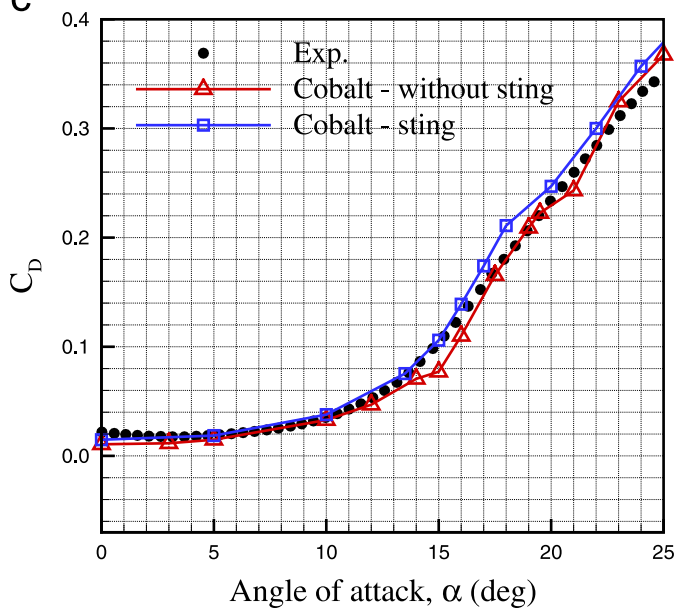

b

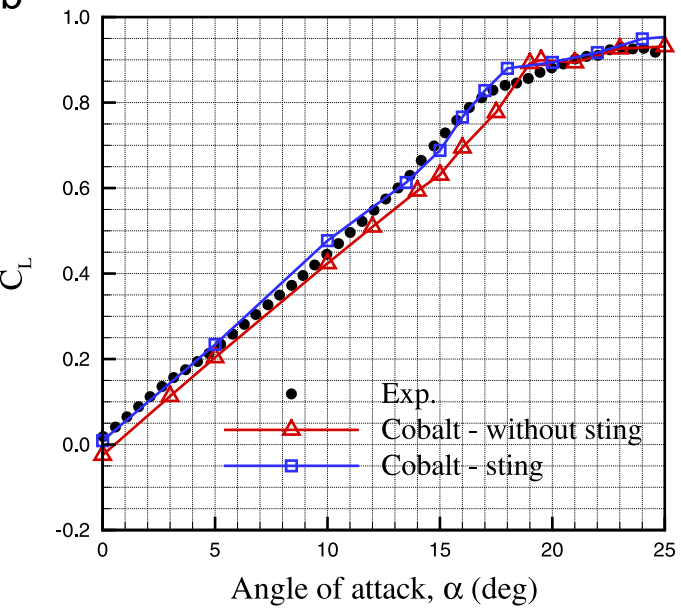

d

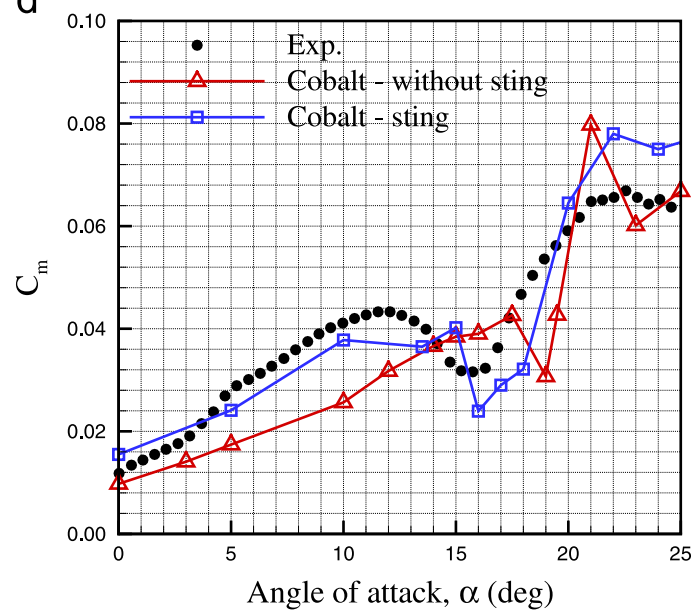

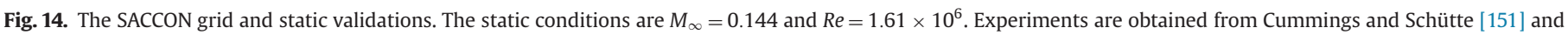

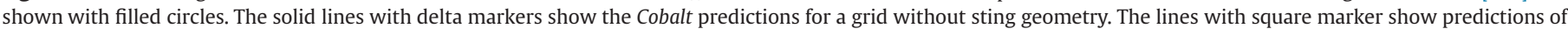

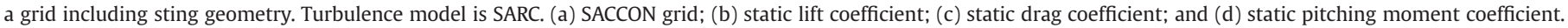

around $4.5 \mathrm{M}$ points and $13 \mathrm{M}$ cells. The total run time of 1000 iterations for the model without sting geometry was $5 \mathrm{~h}$ using 128 processors. The SA with Rotation/curvature Correction (SARC) [153] turbulence model was used for all CFD simulations. The predicted static coefficients of lift, drag, and pitch moment were compared with experiments in Fig. 14. The figures show that CFD predictions closely follow the trends of experimental data up to moderate angles of attack. The offsets in low angle of attack pitch moment in the model have been shown to be due to the effects of the belly sting mounting present in the experiments. For the ROM studies, the mesh without sting geometry was used since it has less grid points.

Some of the SACCON aerodynamic features are shown in Fig. 15. Two emanating vortices from the wing tip and apex are present at $14^{\circ}$ angle of attack (Fig. 15(a)). These vortices lead to a negative pressure region on the upper wing surface, and hence augments the lift force. As the angle of attack increases above $16^{\circ}$, the onset point of the outboard vortex starts to travel toward the wing apex due to increasing adverse pressure gradients. At $19.5^{\circ}$ angle of attack the vortices are already interacting as shown in Fig. 15(b). Further increasing of angle of attack causes the inboard vortex to start to break down (Fig. 15(c)). At higher angles of attack the tip vortex also breaks down. The interaction of the vortices produces a strong recirculation zone over the upper wing (Fig. 15(d)) and results in wing stall and the aerodynamic center backward movement.

For generation of 6DoF SACCON maneuvers, the wind tunnel model was scaled up to fit the characteristics of a real aircraft. Initially, estimations of the mass and moments of inertia were made, through work carried out in the NATO group, based on the Northrop YB-46 aircraft. Table 3 summarizes the SACCON geometry parameters and mass and moments of inertia.

\section{4. $X-31$}

The X-31 aircraft is considered in this paper as well. The aircraft geometry and wind tunnel data were again provided to the 
a

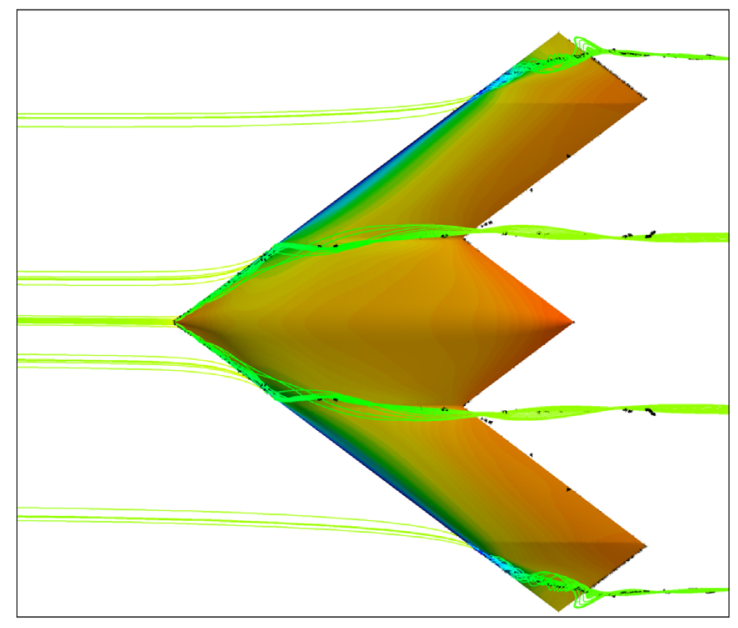

C

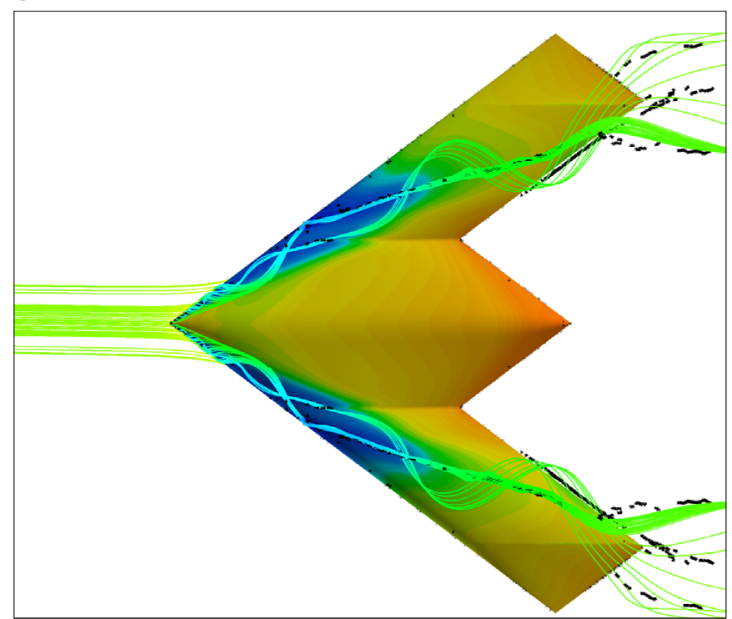

b

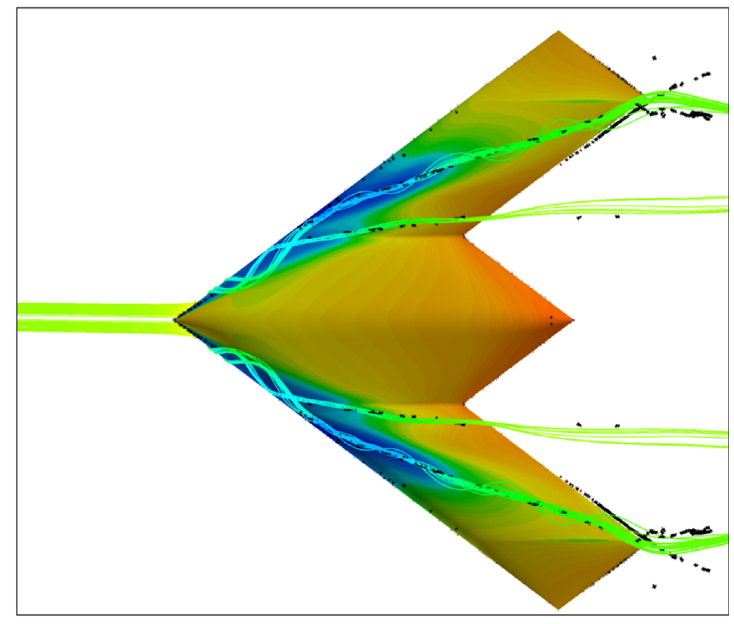

d

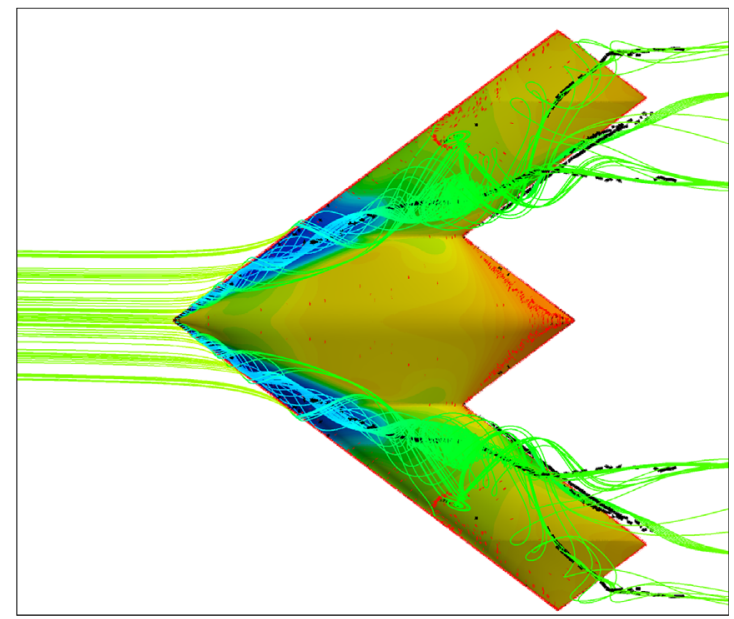

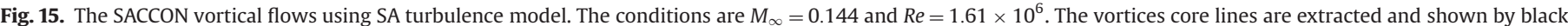

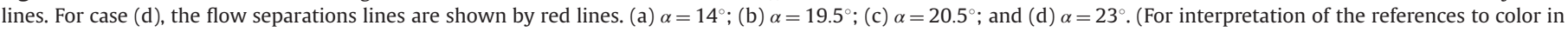
this figure caption, the reader is referred to the web version of this paper.)

Table 3

Geometry parameters and mass/inertias of SACCON flyable model.

\begin{tabular}{lc}
\hline Mean aerodynamic chord, $\bar{c}(\mathrm{~m})$ & 5.011 \\
Wing area, $S\left(\mathrm{~m}^{2}\right)$ & 55.0 \\
Wing span, $b(\mathrm{~m})$ & 13.0 \\
Ixx $\left(\mathrm{kg} \mathrm{m}^{2}\right)$ & 8014 \\
Iyy $\left(\mathrm{kg} \mathrm{m}^{2}\right)$ & 6564 \\
Izz $\left(\mathrm{kg} \mathrm{m}^{2}\right)$ & 8937 \\
Maximum take-off weight, MTWO & 2000 \\
$\quad(\mathrm{~kg})$ & \\
\hline
\end{tabular}

participants in NATO RTO Task Group AVT-161. The vehicle is a super-maneuverable fighter which was built by the United States and West Germany in the 1990s. The test aircraft has been a subject of extensive flight and wind tunnel tests (see for example [154-157]), as well as CFD simulations (an example is the work of Schütte et al. [11]). A three-view drawing of the vehicle is shown in Fig. 16. The aircraft has a fuselage length of $13.21 \mathrm{~m}$, a canard, and a double delta wing with total wing span of $7.26 \mathrm{~m}$. The inner delta wing has a sweep angle of $57^{\circ}$ and the outer sweep is $45^{\circ}$. The inner wing sweep places the wing behind the supersonic shock wave, while the outer one improves the vehicle stability and control [158]. The canard is a cropped delta wing with a sweep angle of $45^{\circ}$. Additional characteristics of the model are the inner

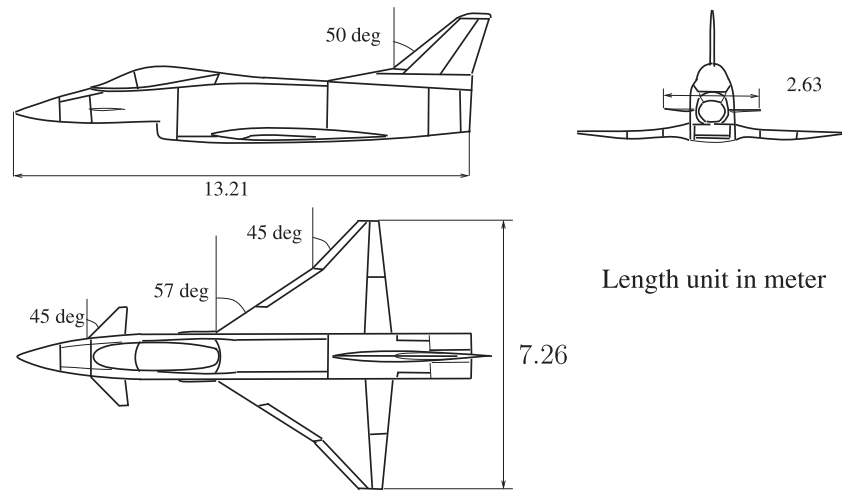

Fig. 16. The X-31 aircraft geometry.

and outer leading edge flaps, the trailing edge flaps, the front wing, and rear fuselage strakes.

The mesh overview is shown in Fig. 17. The grid is a symmetric configuration and contains 4.9 million points and 11.7 million cells. Three boundary conditions were imposed to the surfaces: a farfield, symmetry, and solid wall. The low-speed experiments were made available by the DLR, German Aerospace Center [157]. The wind tunnel model has a closed inlet and is fitted with moving lift and control surfaces. The experiments are composed of two 
a

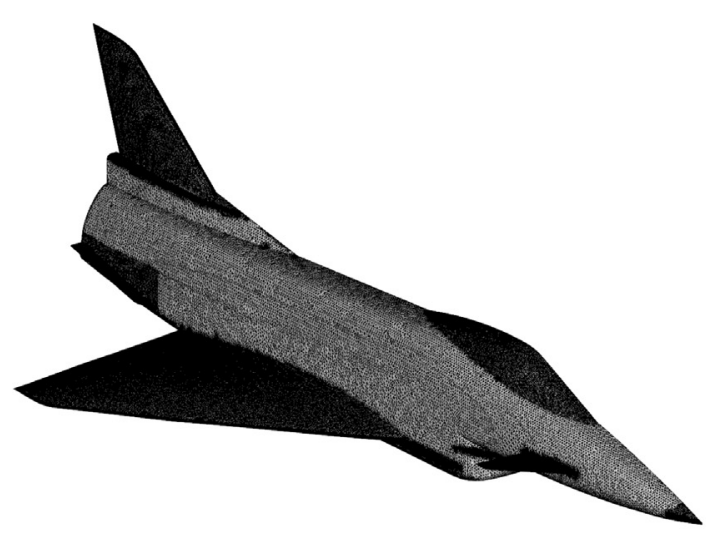

b

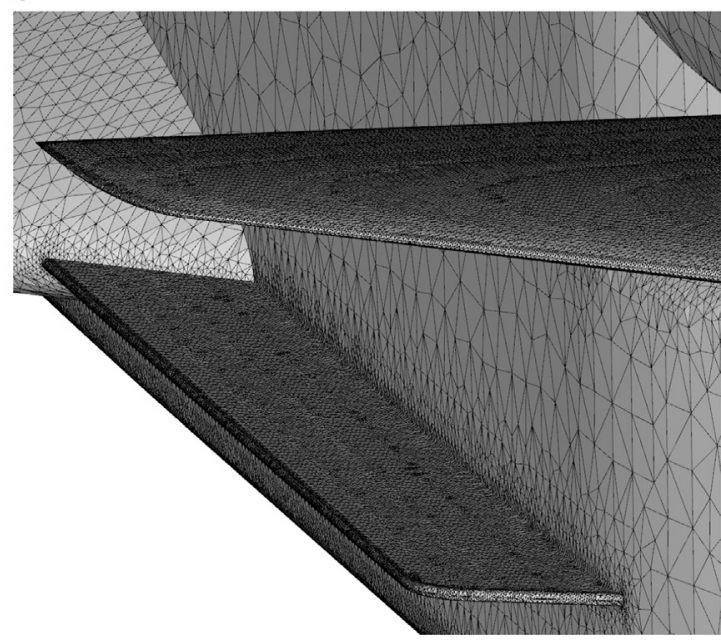

Fig. 17. The X-31 aircraft mesh model. (a) Half-Model Mesh and (b) Surface mesh around LEX and canard.

a

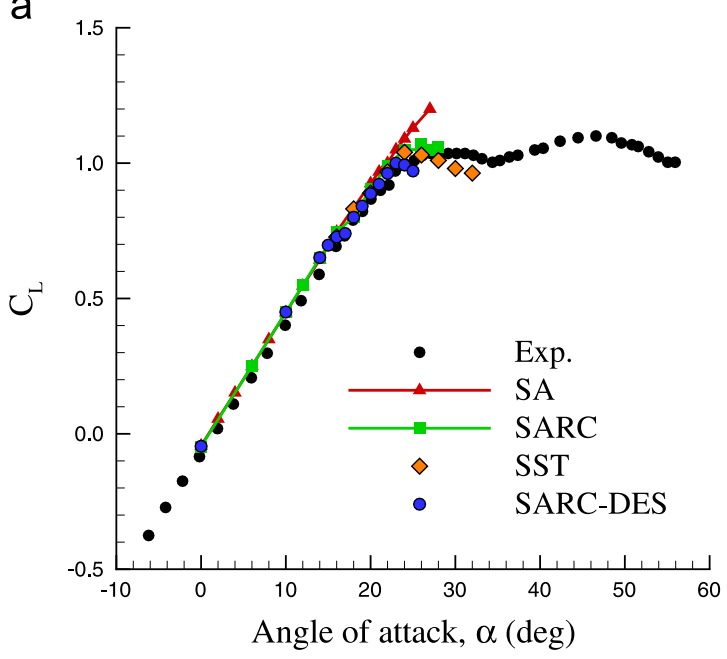

C

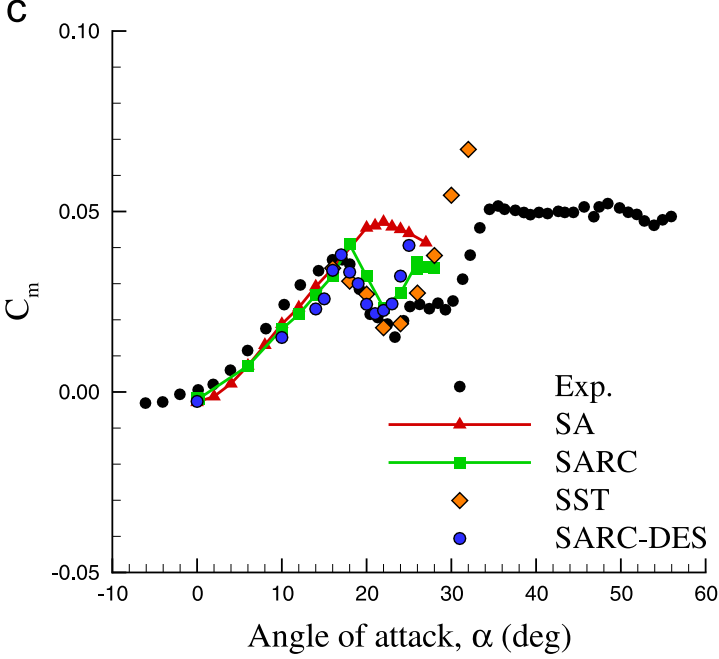

b

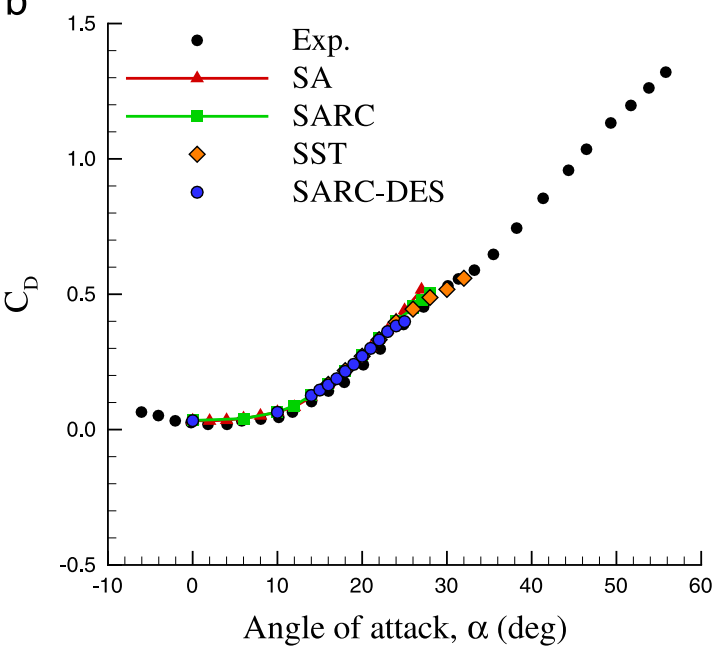


a

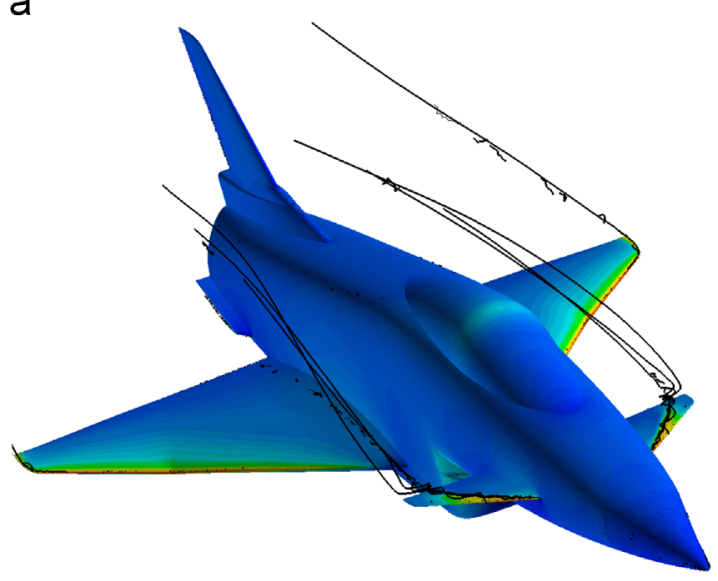

C
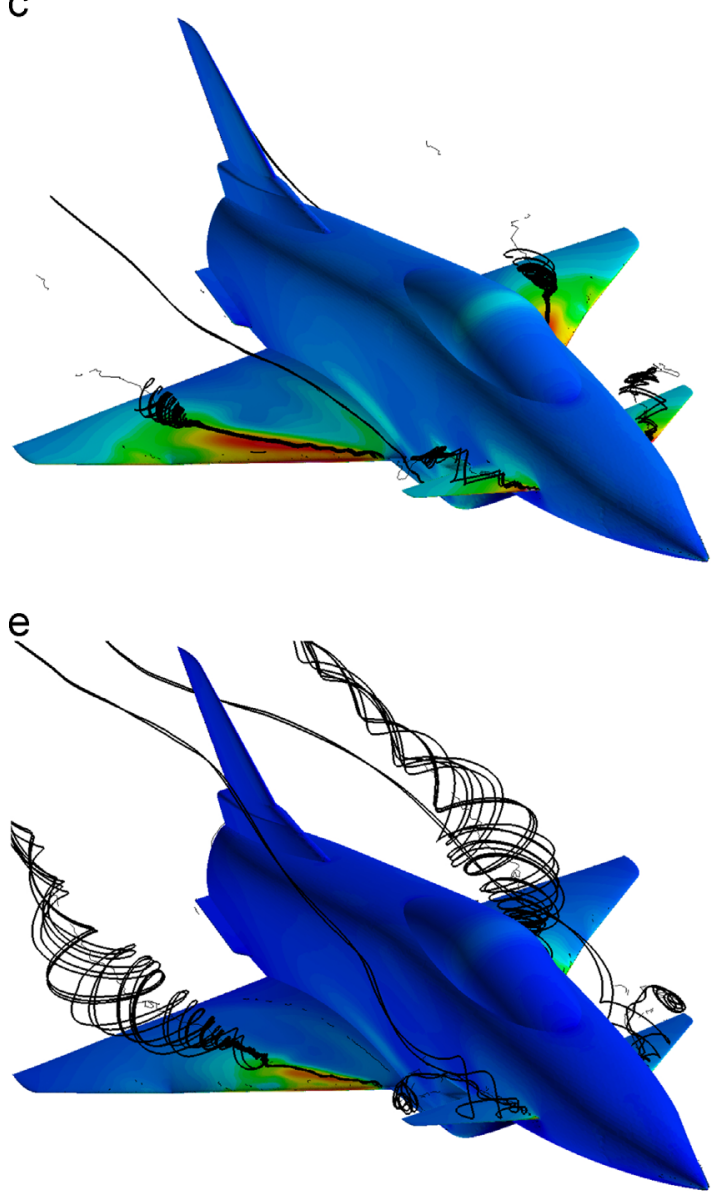

b

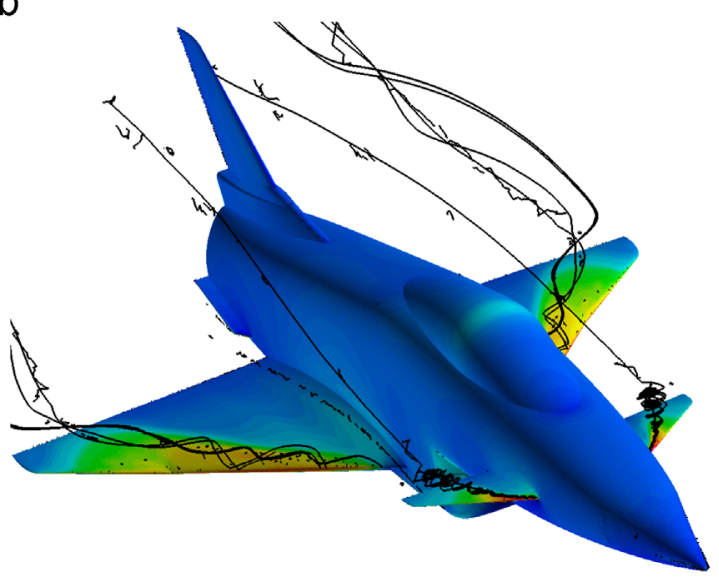

d
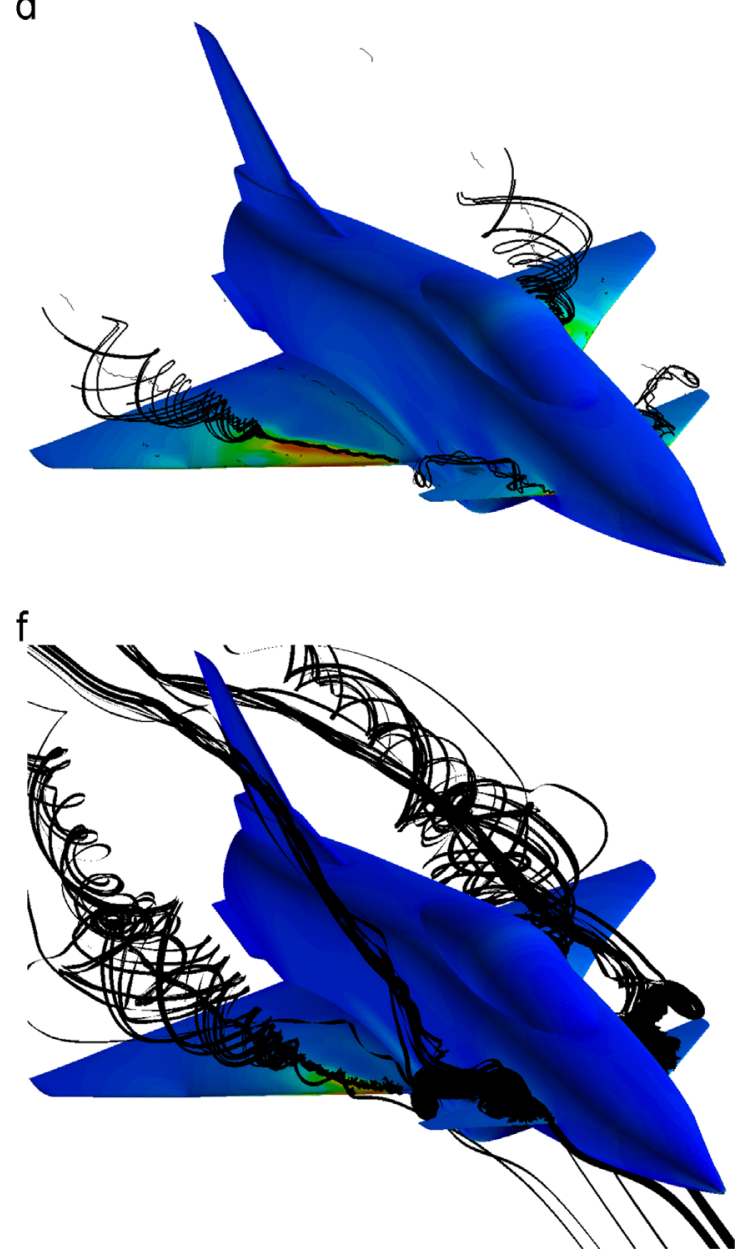

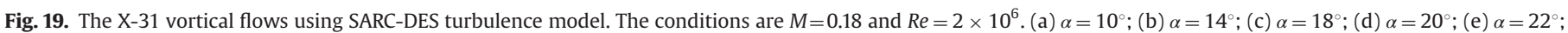
and (f) $\alpha=25^{\circ}$.

attack, but they resulted in a wide spread of predictions at moderate to high angles. For angles between $\alpha=15-23^{\circ}$ SARCDES and SST models performed quite well compared to SA and SARC. DES and SST accurately predicted unsteady separated flows occurring at these angles, but for angles above $23^{\circ}$ all models failed to predict accurately the massively separated flows.

Some features of aerodynamic characteristics from the SARCDES turbulence model predictions are explained. There is an emanating vortex from the canard tip at small angles of attack. This vortex is the source of the small non-linearity in the pitch moment at low angles of attack. As the angle of attack is increased, the canard vortex becomes stronger, resulting in a negative pressure on the upper surface and forward movement of the aerodynamic center. Therefore, the pitch moment slope suddenly increases from the slope value at zero degrees angle of attack. This vortex is shown in Fig. 19(a) for $10^{\circ}$ angle of attack. Around an angle of attack of $14^{\circ}$, the canard vortex starts to breakdown and the wing vortex is formed as shown in Fig. 19(b). The wing vortex helps to further forward movement of aerodynamic center and increase of pitch moment. At $18^{\circ}$ angle of attack, the canard vortex 
breakdown point is nearly moved to the leading edge and then the wing vortex starts to breakdown as shown in Fig. 19(c). This results in an aft movement of the aerodynamic center and a change in the pitch moment slope sign. The wing vortex breakdown point moves towards the leading edge by increasing angle of attack (Fig. 19(d), (e)). The canard vortex is fully separated at these angles. As the vortex breakdown point becomes close to the wing leading edge (Fig. 19(f)), the pitch moment starts to rise again.
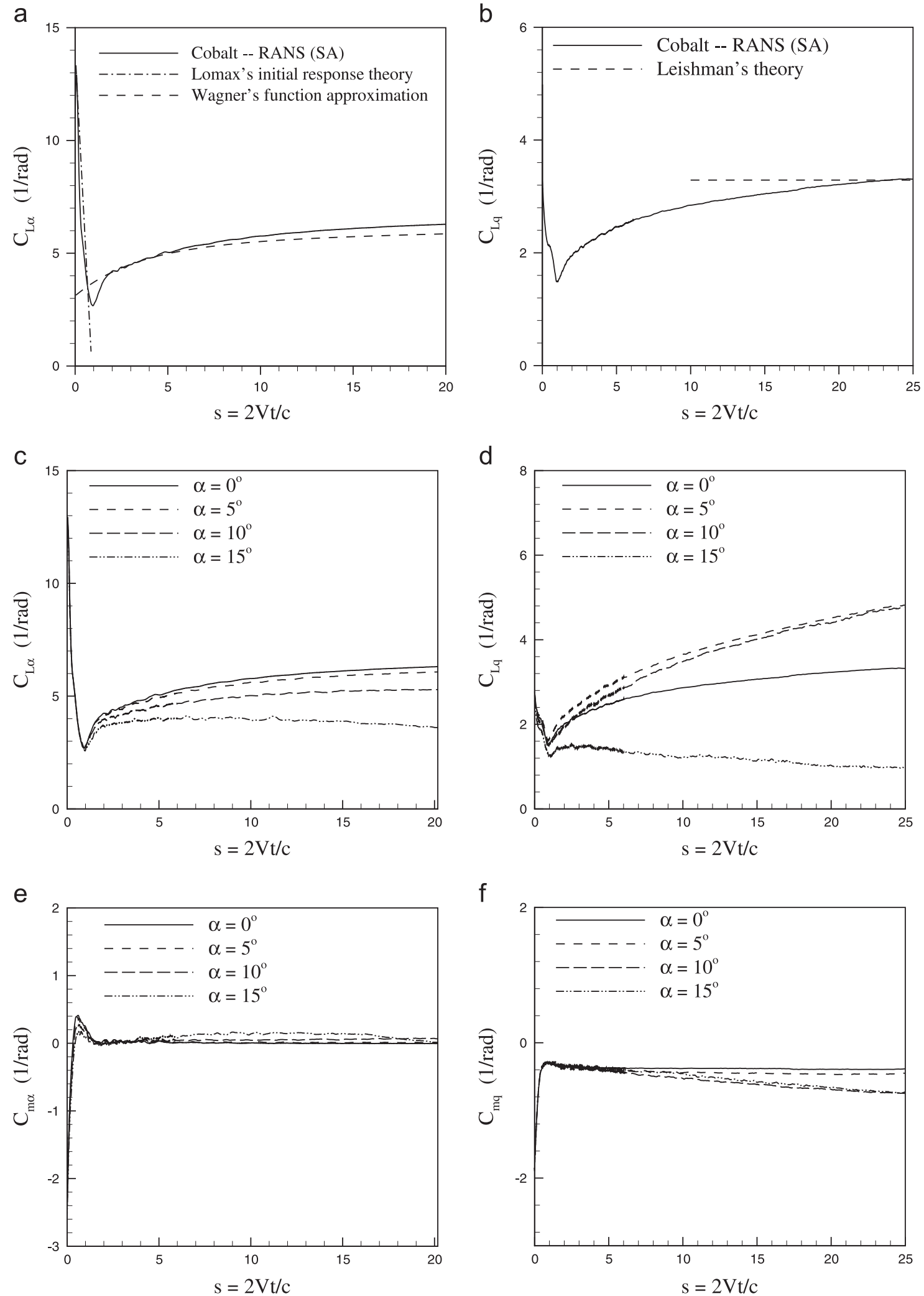

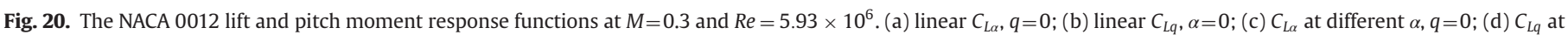
different $\alpha$; (e) $C_{m \alpha}$ at different $\alpha, q=0$; (f) $C_{m q}$ at different $\alpha$. 


\section{Analysis}

The models are evaluated by comparing the predictions with time-accurate CFD solutions which are labeled "Time-Marching" in the plots. The prediction capability of ROMs is reviewed first for the airfoil, SDM, and SACCON test cases. These models are then tested for a pitching motion of the X-31 configuration to permit direct comparison of costs and errors. In this work, the error norm was defined as

$E=\frac{\sum_{i=0}^{N}\left|y^{\mathrm{cfd}}[i]-y^{\mathrm{rom}}[i]\right|}{y_{\max }^{\mathrm{cdd}}-y_{\min }^{\mathrm{cdd}}} \cdot 100$

where $N$ is the total number of time-steps used in the CFD solutions, and the superscripts cfd and rom indicate, respectively, the time-marching and the model predictions .

\subsection{Airfoil aerodynamics modeling}

All airfoil calculations were run on 10 cores with $2.3 \mathrm{GHz}$ core speed. The ROMs reviewed for the airfoil predict only lift and pitch moment coefficients and were used at a fixed Mach number similar to the one used in the training maneuver/response function calculations.
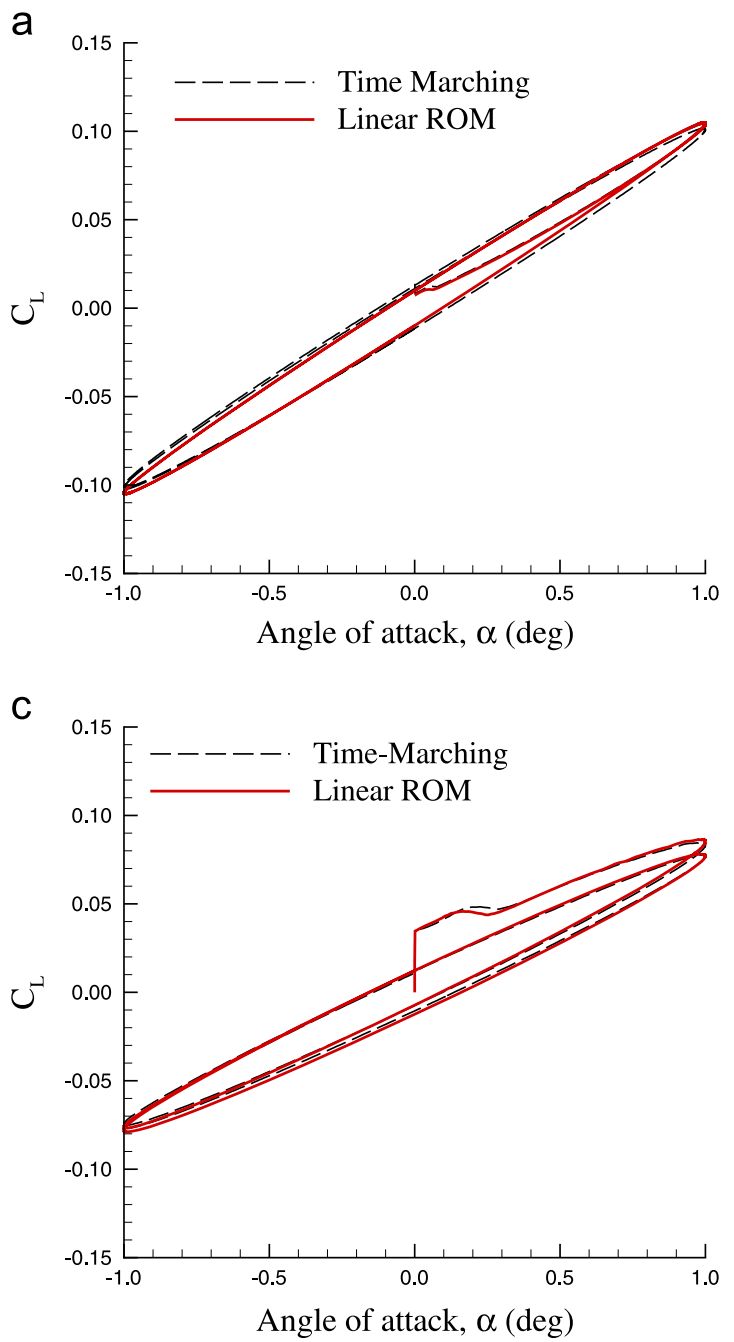

\subsubsection{Models for $M=0.3$}

The RANS grid with the SA turbulence model was used for the response calculations of the NACA 0012 airfoil. The step responses of the airfoil are shown in Fig. 20 for $M=0.3$. These simulations were run for $0.125 \mathrm{~s}$ with a time step of $5 \times 10^{-5} \mathrm{~s}$. Fig. 20(a) and (b) shows that the lift has a peak at $s=0$ followed by a rapid falling trend. The lift again builds up and asymptotically reaches the steady-state value at the final time. The initial peak can be explained based on the energy of acoustic wave systems created by the initial perturbation $[89,106]$. Fig. 20 shows that the lift values at the initial and final times of the response are favorably comparable with the analytical results of Lomax, Wagner, and Leishman. Fig. 20 also shows the effects of angle of attack on response functions. The results show that initial values of the response functions are invariant with the angle of attack but the transient trend and steady state values change depending on the angle of attack.

A linear ROM was created using Eq. (23) and indicial responses at zero degrees angle of attack. This model was then used for the response prediction of two pitching motions with reduced frequencies of $k=0.077$ and 0.307 , a mean incidence of zero degrees, and an amplitude of one degree. The results of the linear model are compared with the time-marching solutions in Fig. 21. The comparisons show that the linear ROM provides very good agreement for these slow and fast motions. The error norm values
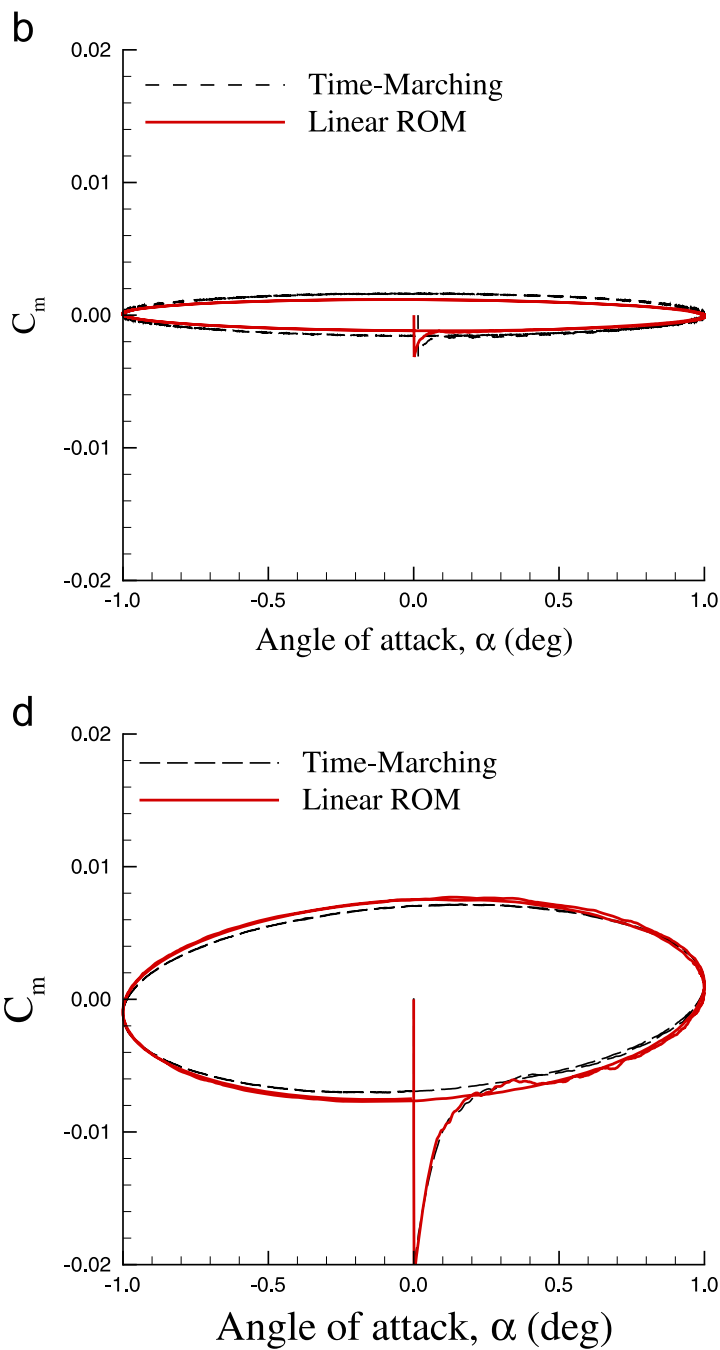

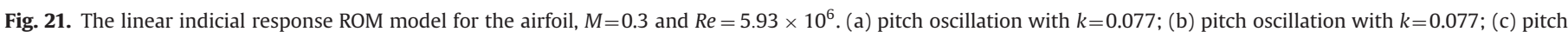
oscillation with $k=0.307$; and (d) pitch oscillation with $k=0.307$. 
for the lift and pitch moment coefficients are around $0.9 \%$ and $2.2 \%$, respectively.

A nonlinear ROM was also created using Eq. (24) and nonlinear response functions calculated at $M=0.3$. Fig. 22(a) and (b) compares the linear and nonlinear ROM predictions for a ramp increase of
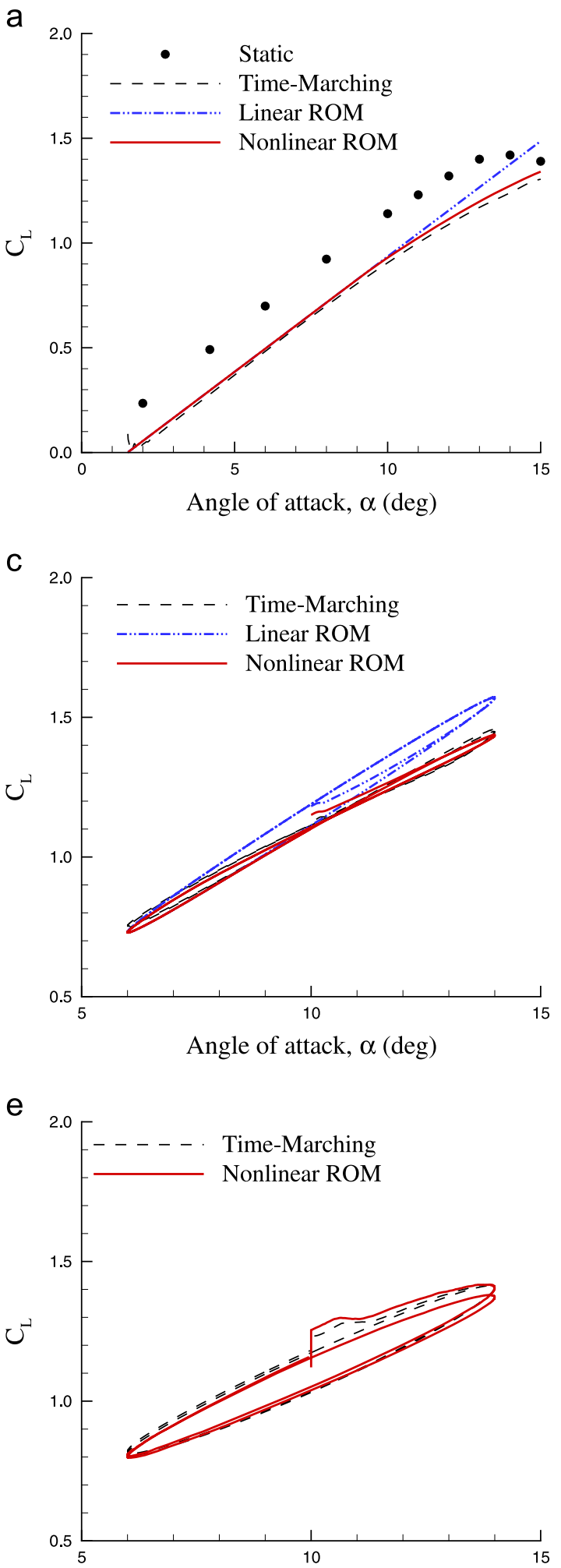

Angle of attack, $\alpha$ (deg) angle of attack from $1.5^{\circ}$ to $15^{\circ}$ with a rate of $10 \mathrm{deg} / \mathrm{s}$. The grid is undergoing only a translational motion, so the second terms in Eqs. (23) and (24) are zero. Notice that the forces and moments acting on the airfoil during translation are different from the static values. The flow change is not as fast as the angle of attack change and hence

b

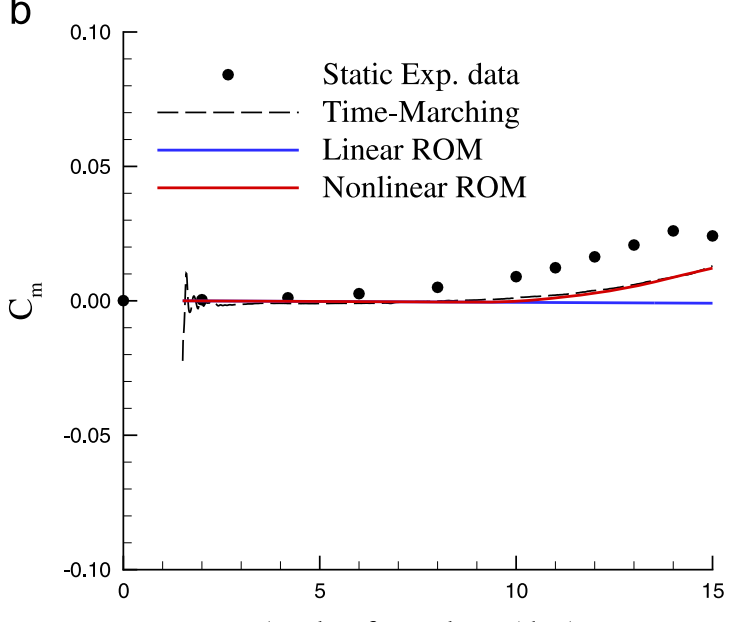

Angle of attack, $\alpha(\mathrm{deg})$

d

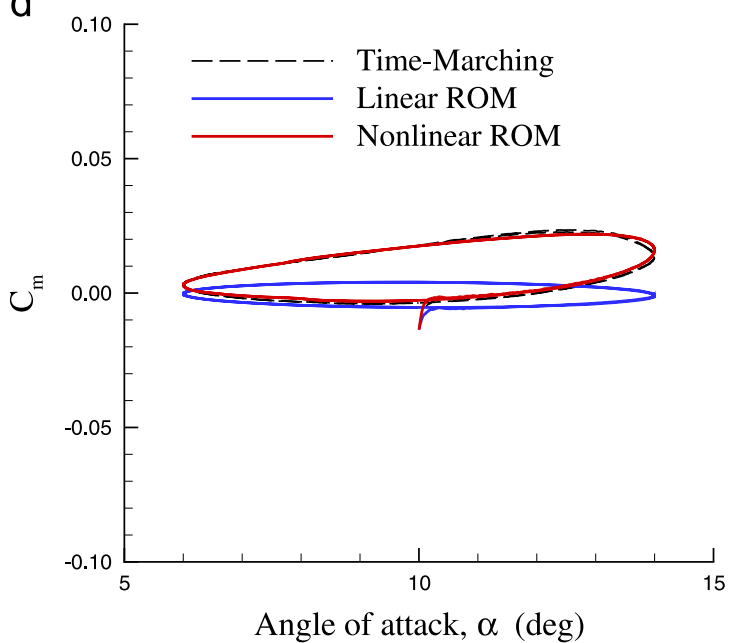

$f$

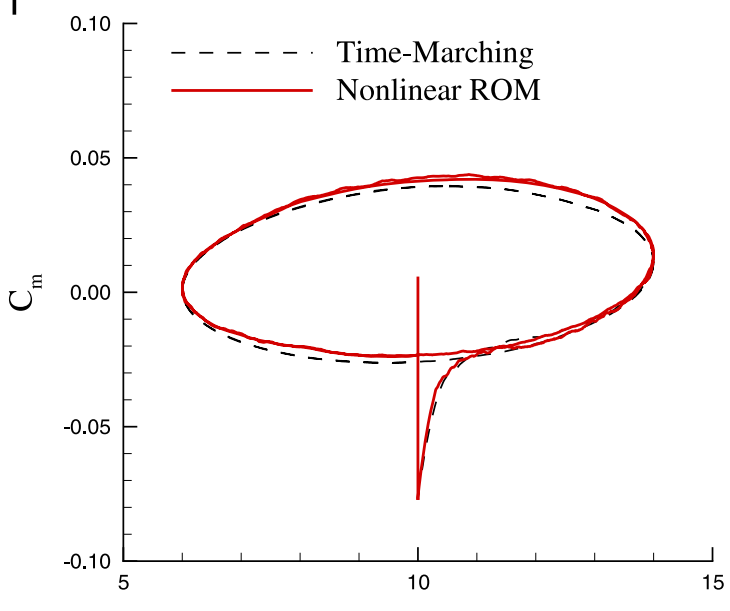

Angle of attack, $\alpha(\mathrm{deg})$

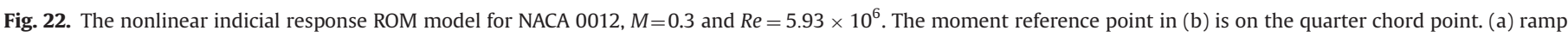

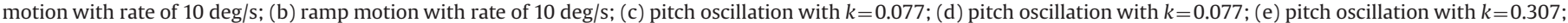
and (f) pitch oscillation with $k=0.307$. 
the ramp motion underestimates the static coefficients. The results show that for low angles of attack both linear and nonlinear ROMs are in good agreement with values from the time-marching simulation. For large angles of attack, the nonlinear model closely follows the time-marching solution, but the linear model is off since the model formulation is valid in linear regimes. The error norm values of the linear model are $2.82 \%$ and $5.0 \%$ for the lift and pitch moment coefficients. These errors drop to $1.4 \%$ and $2.2 \%$ using the nonlinear model.

Fig. 22(c)-(f) also shows two pitch oscillation cases with reduced frequencies of $k=0.077$ and 0.307 . Both motions start from $\alpha=10^{\circ}$ and have an amplitude of $4^{\circ}$. Fig. 22(c)-(f) shows that the nonlinear ROM predictions agree well with time-marching simulation data. The error norm values are around $2.3 \%$ and $2.7 \%$ for the lift and pitch moment coefficients, respectively.

Some of the model strengths and weaknesses are now briefly highlighted. An advantage of the indicial response model is that responses are calculated as a function of time and include the effects of $\dot{\alpha}$ and $\dot{q}$, therefore they can be used for any frequency of interest. These models can also predict the initial transient behavior seen in CFD solutions. However, response functions do not work for the full range of Mach numbers. For aerodynamic modeling of large amplitude motions, two types of problems were also reported [159]: (1) a large number of simulations is required

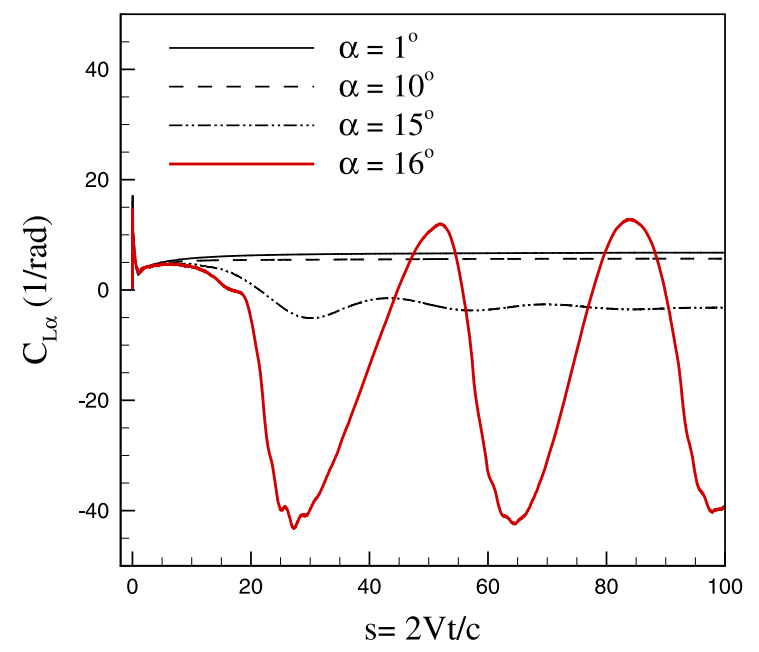

Fig. 23. The NACA 0012 airfoil angle of attack response functions. In these simulations $M=0.3$ and $R e=5.93 \times 10^{6}$.

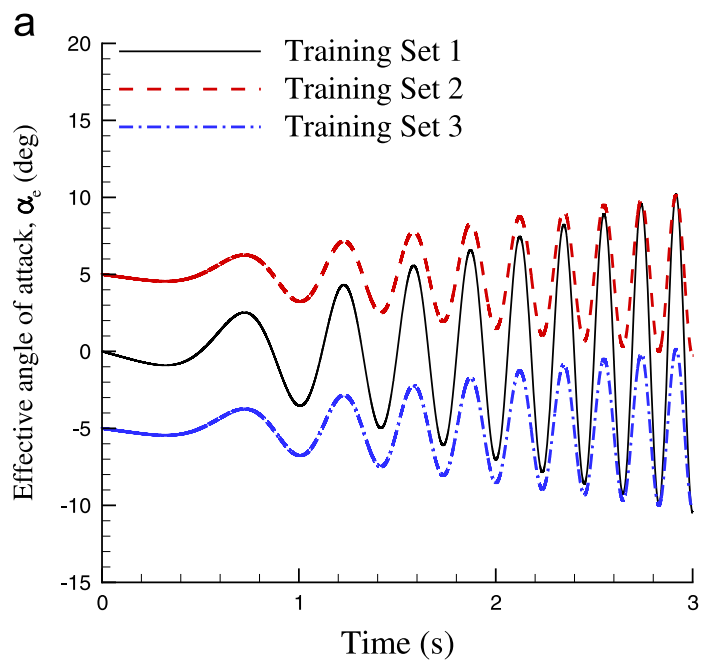

to model a highly nonlinear motion, therefore, the reduced order model becomes quiet expensive to generate, and (2) the second problem is related to the stability of response type CFD calculations at large angles of attack. Fig. 23 shows the effects of angle of attack on the responses of the NACA 0012 airfoil at $M=0.3$. Fig. 23 shows that as the angle of attack reaches the stall angle, small oscillations are found in the response solutions. For angles of attack beyond the stall angle, the oscillations become large and persist for a long time. This behavior makes the nonlinear indicial functions infeasible to model motions at large incidence angles.

In terms of computational cost, the linear and nonlinear models required about 0.6 and $6.0 \mathrm{~h}$ of CPU time, respectively. The nonlinear model requires response calculations at different angles of attack and hence comes with more computational cost. However, once these models are constructed, they can be used to predict airfoil responses to a wide range of pitching and plunging motions in order of a few seconds. In comparison to model costs, each time-accurate simulation (full-order model) costs around $1.8 \mathrm{~h}$ of CPU.

RBF neural networks were also used for aerodynamic modeling of the NACA 0012 airfoil [36]. The networks used had a hidden radial basis layer with a Gaussian transfer function. All training data were generated using CFD with either the Euler or RANS equations. Six training datasets were defined to find a mapping

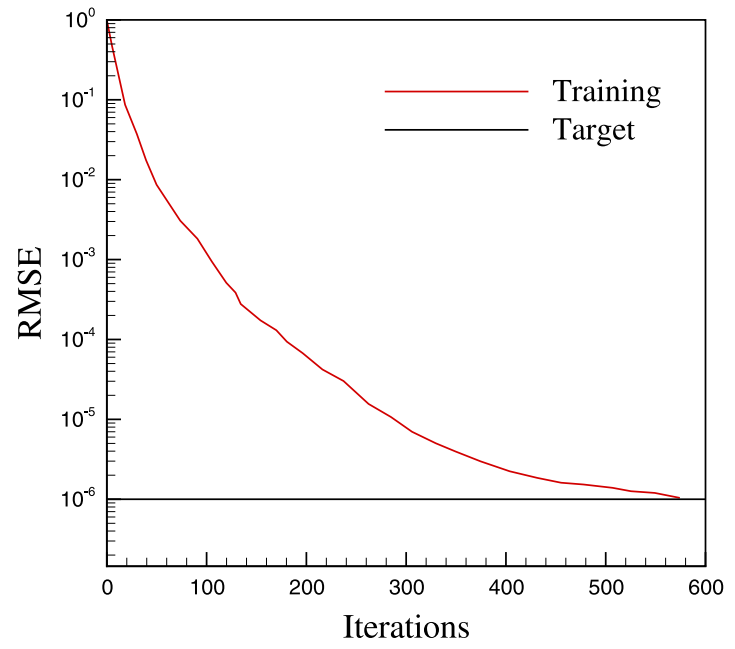

Fig. 25. RBF neural network training performance. The $y$-axis displays root mean squared error and the $x$-axis shows number of iterations.

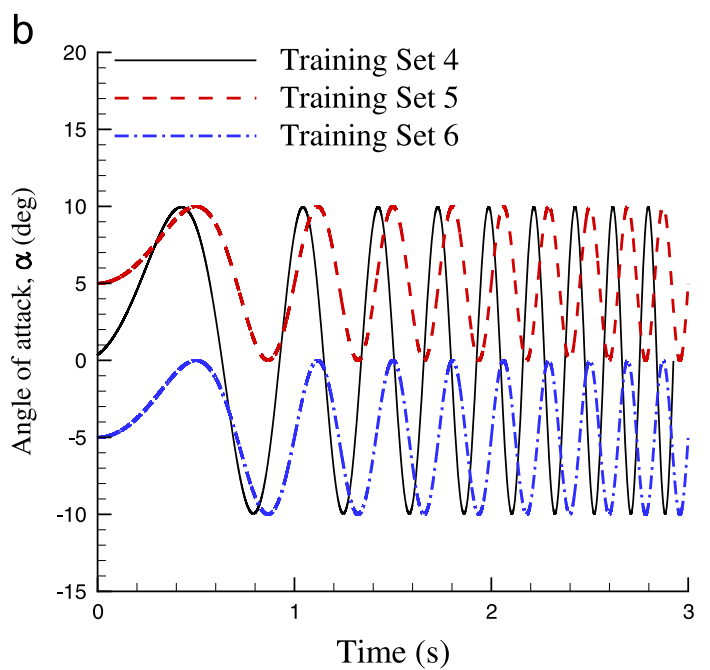

Fig. 24. The plunge and pitch training maneuvers for airfoil Euler simulations. (a) plunge training motions and (b) pitch training motions. 

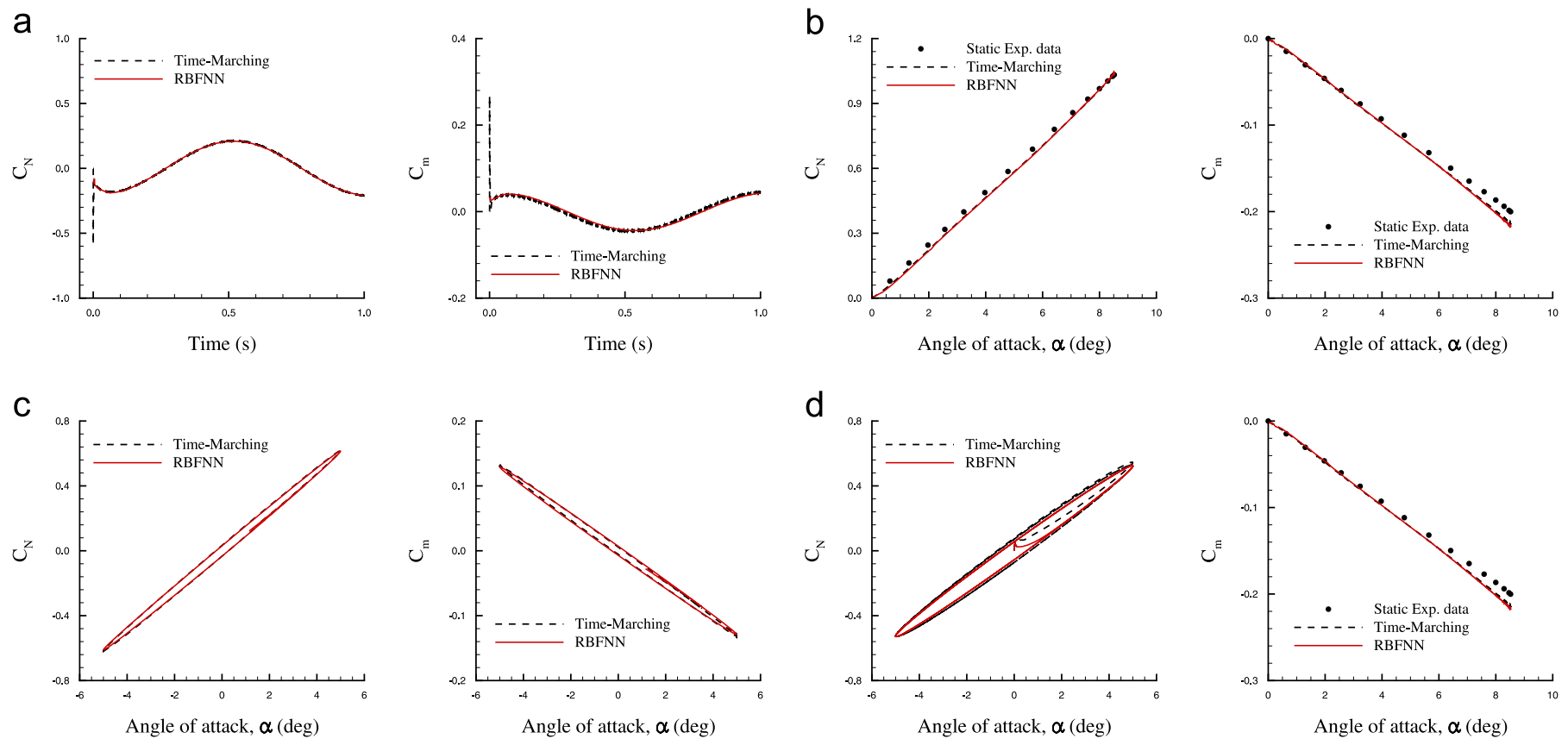

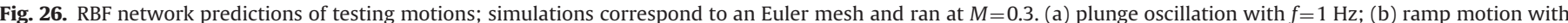
rate of $10 \mathrm{deg} / \mathrm{s}$; (c) plunge oscillation with $f=0.5 \mathrm{~Hz}$; and (d) pitch oscillation with $f=2.5 \mathrm{~Hz}$.
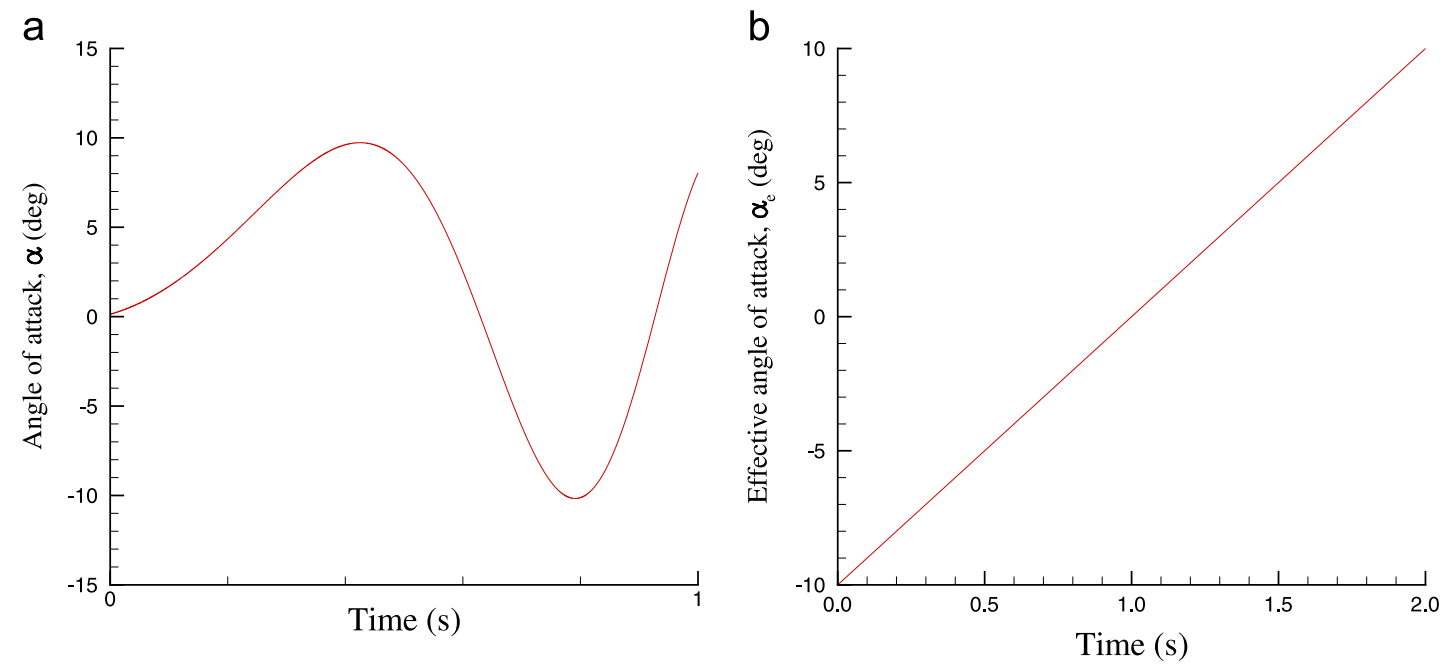

Fig. 27. Training data for the airfoil RANS simulations; $M=0.3$. (a) pitching motion and (b) ramp motion.

a

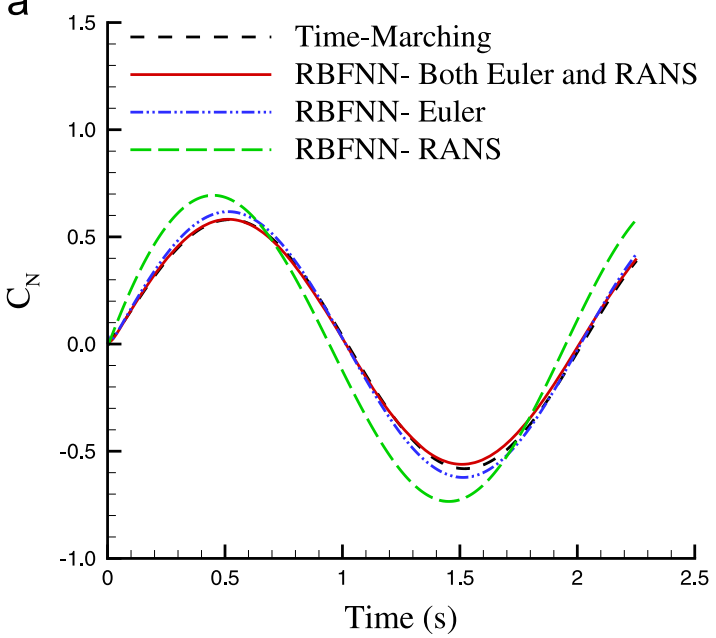

b

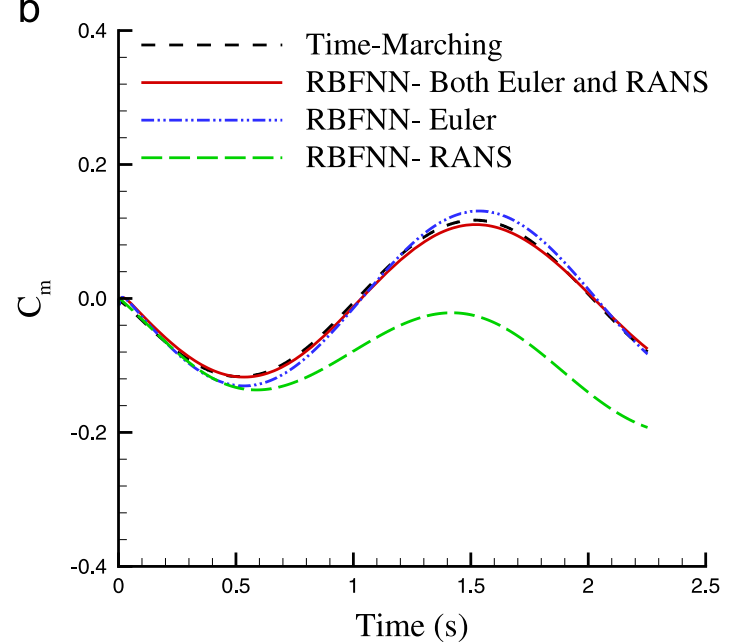

Fig. 28. RBFNN predictions of the airfoil pitching motion using both Euler and RANS equations; $M=0.3$. (a) normal force coefficient and (b) pitch moment coefficient. 

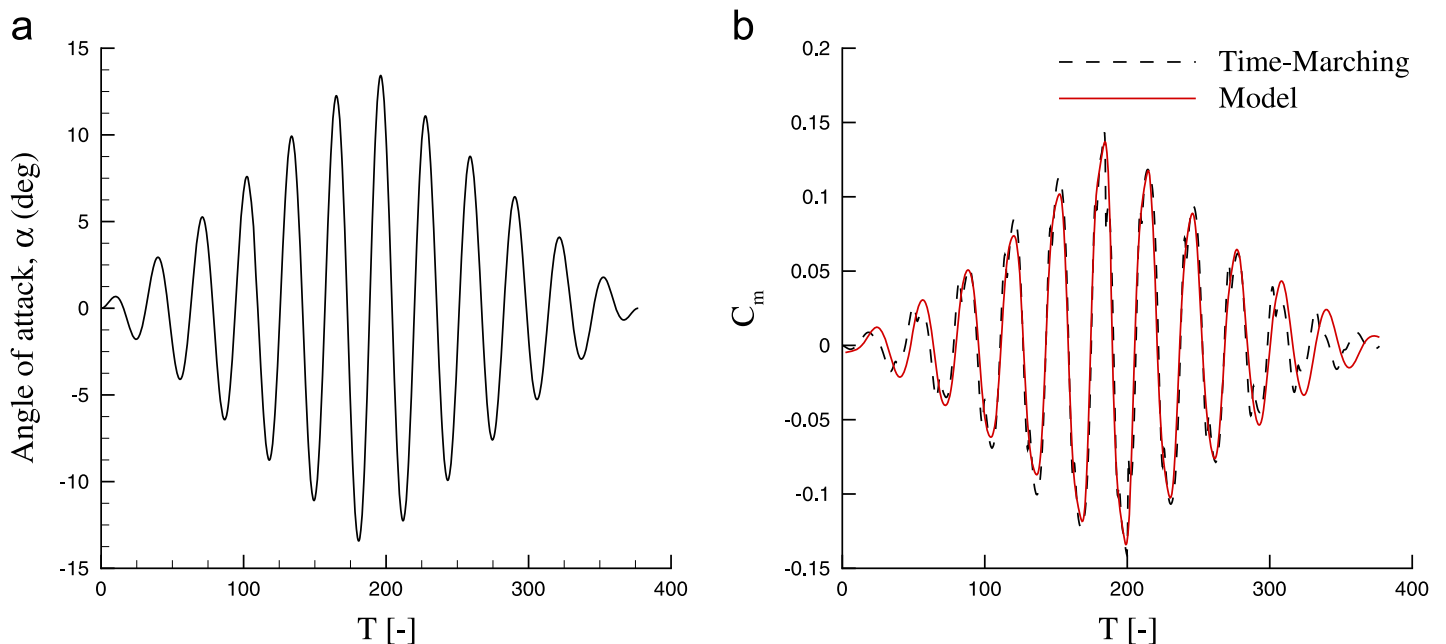

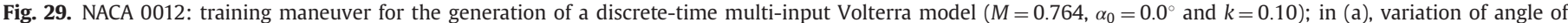
attack with time; in (b), "Model" refers to the discrete-time multi-input Volterra model. (a) angle of attack and (b) pitch moment coefficient.

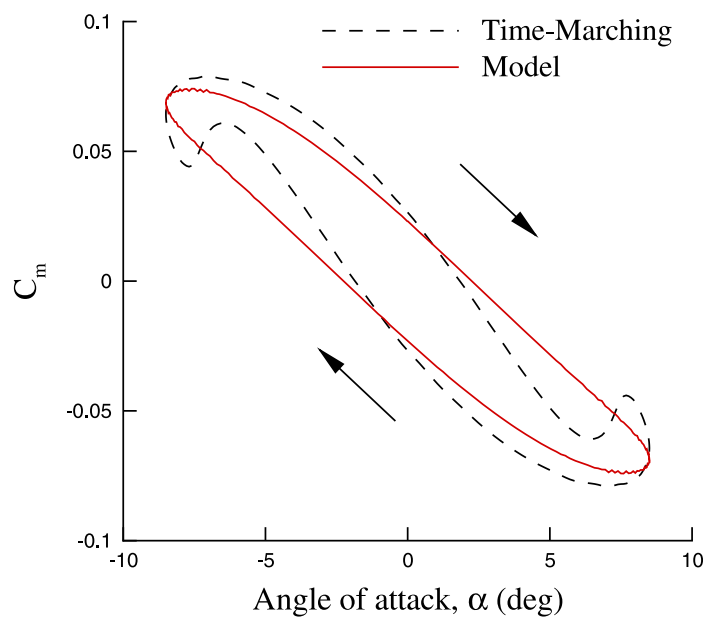

Fig. 30. NACA 0012: predictions of pitching moment dynamic dependence $\left(M=0.764, \alpha_{0}=0.0^{\circ}, \alpha_{A}=8.5^{\circ}\right.$, and $\left.k=0.10\right)$; "Model" refers to the discrete-time multi-input Volterra model.

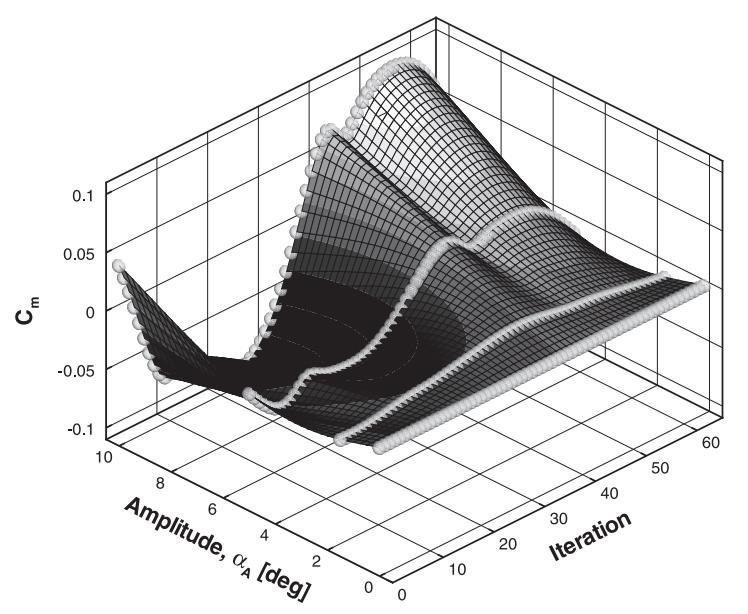

Fig. 31. NACA 0012: carpet plot of the pitching moment coefficient $(M=0.764$, $\alpha_{0}=0.0^{\circ}$, and $k=0.10$ ); large spheres indicate the 4 CFD solutions used to construct the SBRF model.

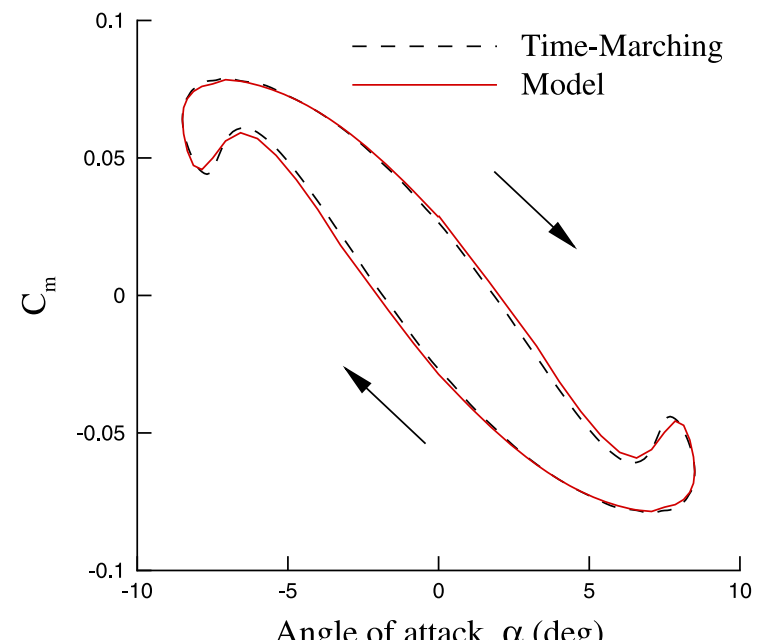

Fig. 32. NACA 0012: predictions of pitching moment dynamic dependence $\left(M=0.764, \alpha_{0}=0.0^{\circ}, \alpha_{A}=8.5^{\circ}\right.$, and $\left.k=0.10\right)$; "Model" refers to the surrogate-based recurrence-framework.

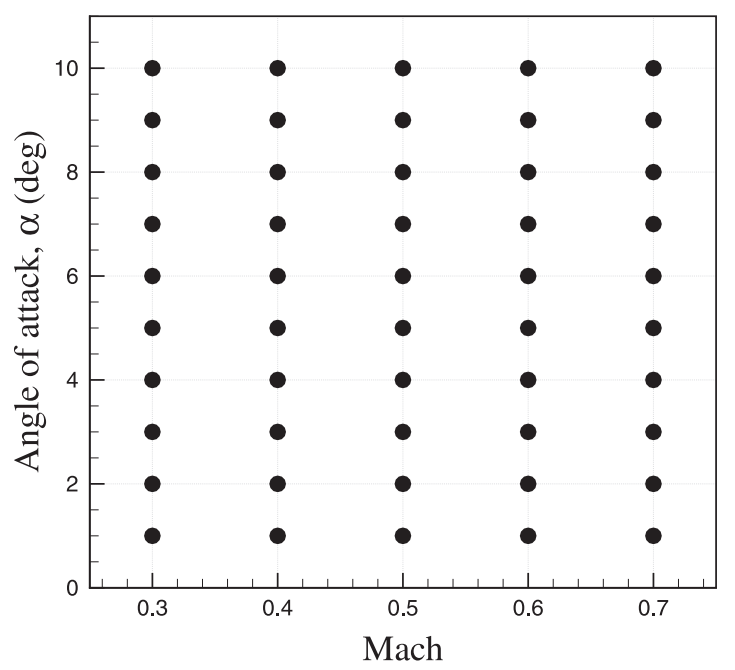

Fig. 33. Design samples. 
a

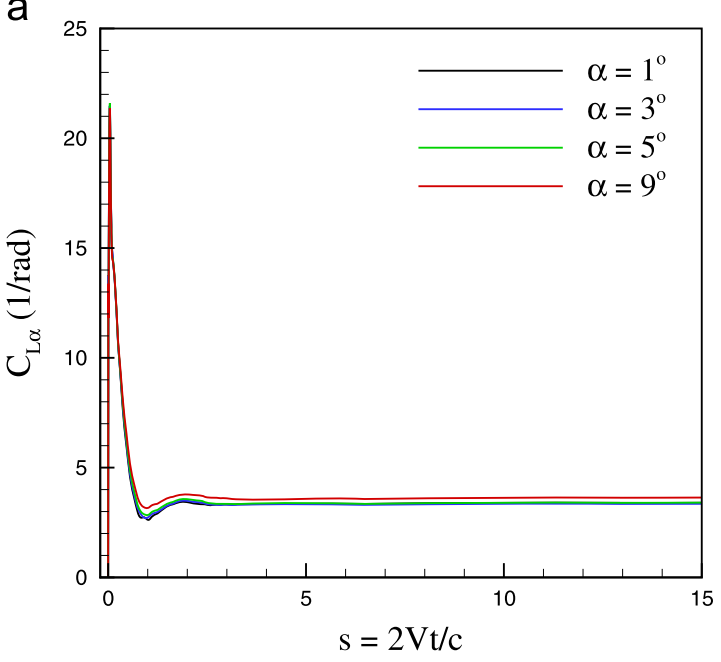

C

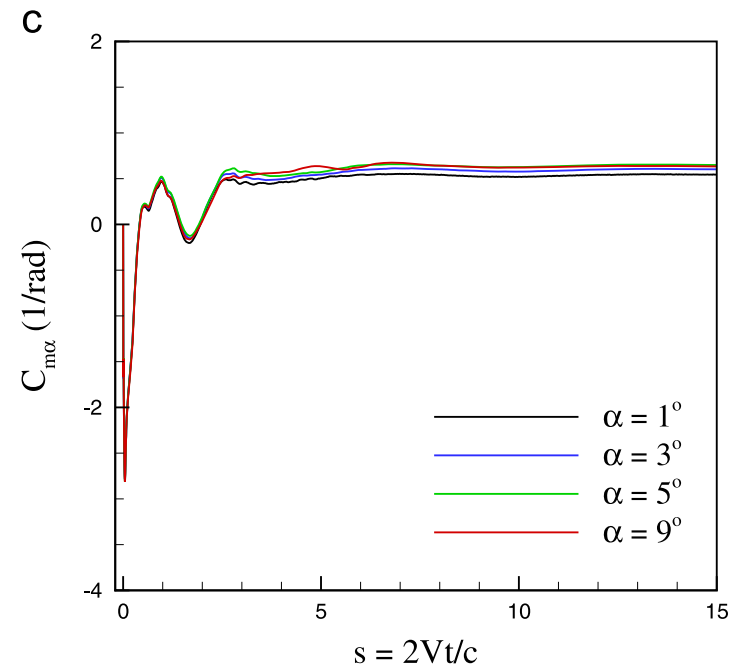

b

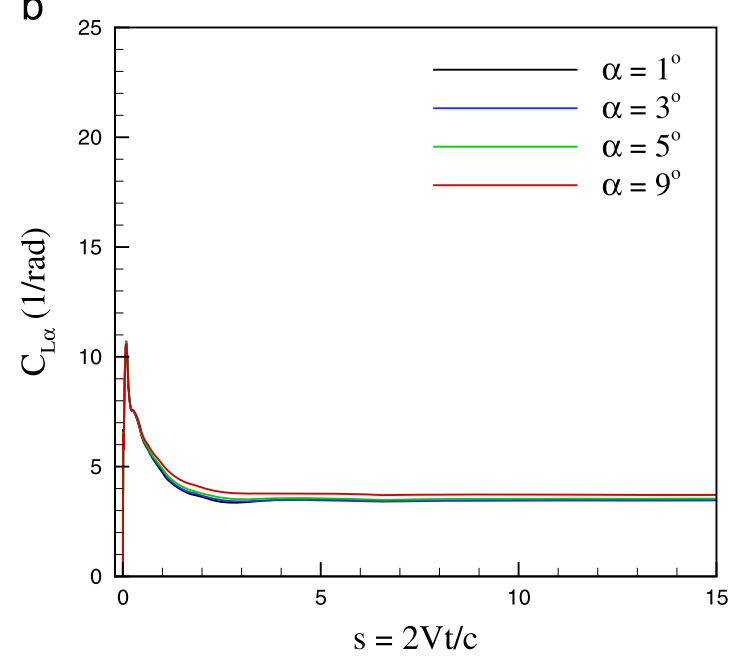

d

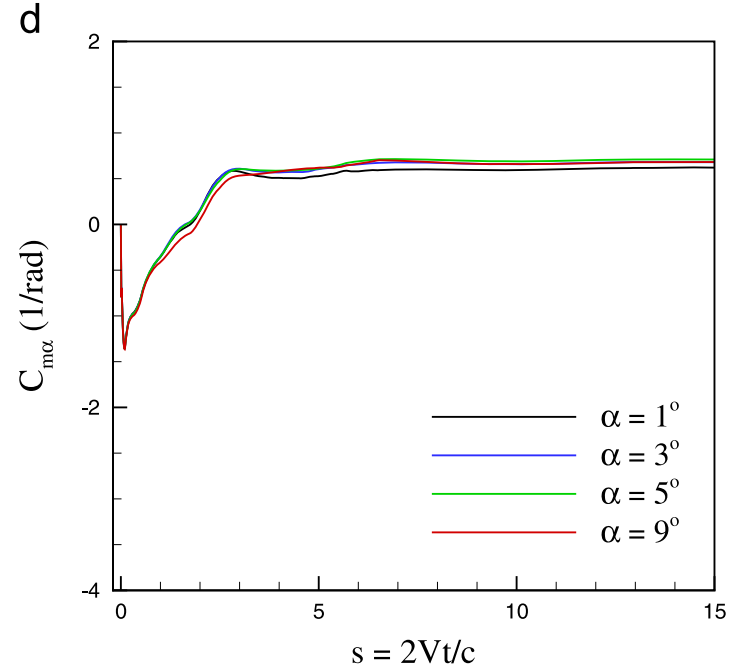

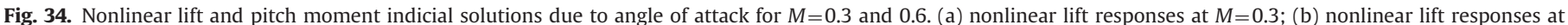
$M=0.6$; (c) nonlinear pitch moment responses at $M=0.3$; (d) nonlinear pitch moment responses at $M=0.6$.

a

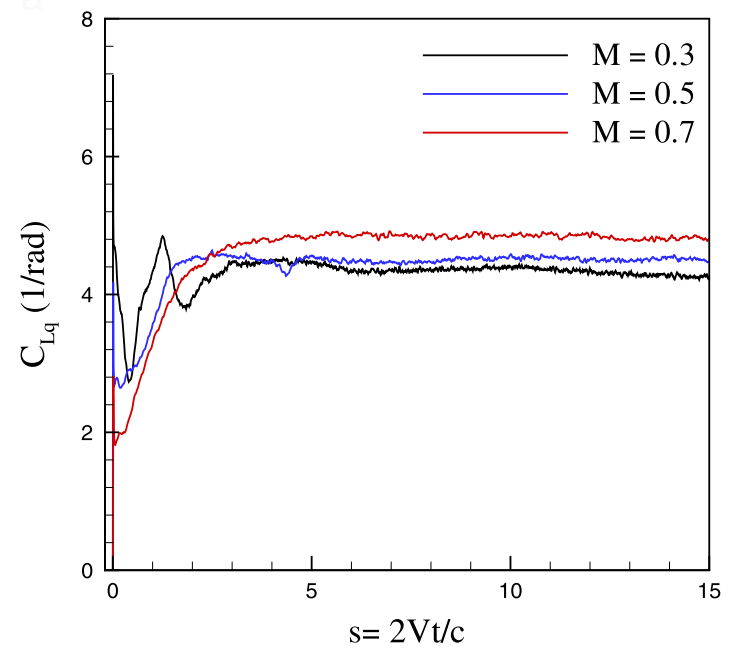

b

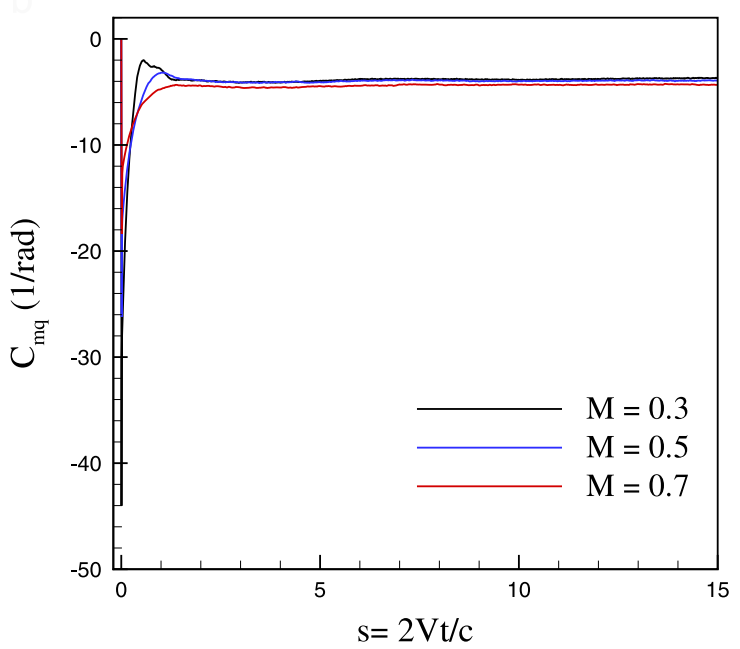

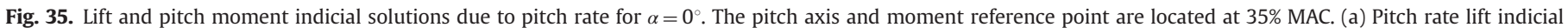
functions and (b) Pitch rate pitch moment indicial functions. 
between aerodynamic coefficients with motion variables at a fixed Mach number of 0.3. These motions are linear chirp type and have an initial frequency of $1 \mathrm{~Hz}$ but started at different angles of attack as shown in Fig. 24. The starting angles of attack are $-5^{\circ}, 0^{\circ}$, and $5^{\circ}$ to include the effects of steady-state conditions into the pitching and plunging motions.

All six training motions were first simulated using the Euler equations. The CFD calculations started from a steady solution and ran for three seconds of physical time with a time step size of $5 \times 10^{-5} \mathrm{~s}$. This gives a total computational cost equivalent to that of 240 Euler steady-state calculations or 17 CPU hours; this is much higher than the cost of response functions because training motions take a longer time to complete than indicial responses. Euler calculations are five times faster than calculations using the RANS grid. Therefore, the cost of simulating all six training motions using the Euler approach is equivalent to that of only 48 RANS steady-state calculations.

The reported normal force and pitch moment coefficients of training maneuvers were re-arranged according to Eq. (30) and then fed to the RBF neural network for training with different values of $n$ and $m$ and with a performance error threshold of $1 \times 10^{-6}$. All networks computed and converged to the threshold error as shown in Fig. 25. The results showed that using $m=2$ and $n=4$ in Eq. (30) is sufficient for modeling the studied motions. The costs to train the networks and that of executing the networks for new maneuvers were around $88 \mathrm{~s}$ and $17 \mathrm{~s}$, respectively.

Once the networks were trained, they were used to predict the aerodynamic loads of new maneuvers again simulated by the Euler equations. The predicted normal force and pitch moment of these maneuvers are compared with CFD data in Fig. 26. Fig. 26 (a) shows the airfoil responses to a plunge oscillation with a frequency of $1 \mathrm{~Hz}$ started at zero degrees angle of attack. The results show that the ROM predictions match well with the timemarching calculations. The error norm values for normal force and pitch moment are $0.75 \%$ and $5 \%$, respectively. The cost of simulating this motion in a time-marching fashion is around 0.36 CPU hours.

Fig. 26(b) compares the ROM predictions for a ramp increase of angle of attack with a rate of $10 \mathrm{deg} / \mathrm{s}$ started at zero degrees angle of attack. The results show that ROM predictions agree well with the CFD prediction (the error norm values are $0.34 \%$ and $5.45 \%$ for the normal force and pitch moment coefficients, respectively). Also, two pitch oscillation motions with frequencies of $f=0.5 \mathrm{~Hz}$ and $f=2.5 \mathrm{~Hz}$ were considered. The low-frequency motion predictions are compared with the CFD data in Fig. 26(c). Again a good match is found (the normal force and pitch moment error norms are $0.46 \%$ and 5.98\%). Likewise, Fig. 26(d) shows that the ROM predictions closely match with the time-marching simulations of the motion with $f=2.5 \mathrm{~Hz}$.

The development of a ROM from RANS/Euler was also investigated by Ghoreyshi et al. [36]. For network training purposes, these data are also called primary/secondary or expensive/cheap data. The cheap data are assumed to provide information at least about the trend of the target function, whereas the expensive calculations give quantitative information. To illustrate the approach the NACA 0012 airfoil was used again. The timemarching Euler predictions of all six training sets described in Fig. 24 are considered as cheap data. It is assumed that these predictions had similar trends to the RANS predictions, but with different values. The new RBF model tries to correct these trends by using much less expensive data.

The expensive (or RANS) calculations were run only for two training sets. The first set is a chirp-pitch that runs for one second of physical time as shown in Fig. 27(a). The second set is a ramp motion with a rate of $10 \mathrm{deg} / \mathrm{s}$, starting at $-10^{\circ}$ and progressing for two seconds of physical time as shown in Fig. 27(b). Both motions have a sweep of angle of attack from the low to high angles, and therefore are found very helpful to learn the differences between Euler and RANS predictions. Both training sets were simulated using time-marching RANS equations and the SA turbulence model. Likewise for the Euler predictions a time step of $5 \times 10^{-5} \mathrm{~s}$ was used. This results in a computational cost equivalent to that of 40 RANS steady-state calculations and a total cost (both Euler and RANS) of 88 RANS steady-state calculations. Note that using RANS for time-marching simulations of all six training sets is equivalent to that of 240 RANS steady-state calculations. In this sense, a ROM generated using both Euler and RANS calculations is $63 \%$ cheaper than a ROM generated using the RANS calculations of six training maneuvers.

The RBFNN was trained for these maneuvers using both RANS and Euler evaluations and then was tested for new maneuvers simulated using RANS equations. A pitch oscillation was used to demonstrate the network performance. The motion is a constant frequency type with $f=0.5 \mathrm{~Hz}$ started at zero degrees angle of attack. The unsteady normal force and pitch moment coefficients were obtained using simulations of unsteady RANS equations and are shown in Fig. 28(a) and (b). Fig. 28(a) and (b) shows that the output of a network using both Euler and RANS training data match well with time-marching values. Such a network has similar trends as predicted by the network trained from Euler data and then corrects them. The network using only Euler data match time-marching solutions at low angles of attack, but overestimates the values at high angles due to the inviscid assumption. Fig. 28 (a) and (b) shows that using only RANS data of two training sets does not help much to approximate the time-marching values, since these maneuvers are short and do not properly cover the frequency and starting angle of attack space.

In comparison to response function models, a training maneuver that even partially covers the input space will take much longer to simulate in CFD than response functions. RBF based model predictions are also influenced by the training maneuver type and the range of frequency and angle of attack used in the training maneuver as well as the network settings. On the other hand, the RBF based models can be applied for aerodynamic loads prediction in the post-stall regime, assuming an appropriate maneuver was used in the network training.

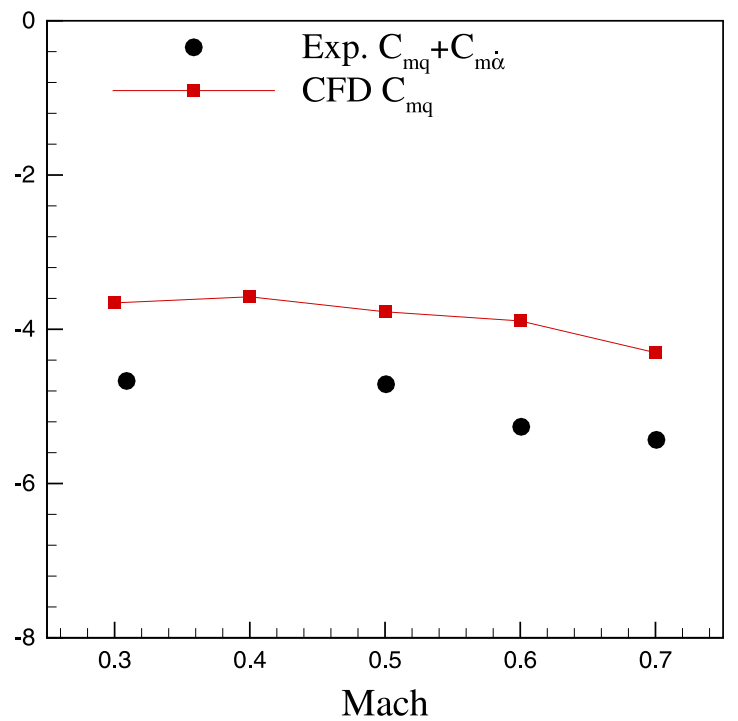

Fig. 36. Validation of $C_{m q}$ values calculated from pitch-rate indicial functions Experimental data are from Da Ronch et al. [161]. 


\subsubsection{Models for $M=0.764$}

Models based on Volterra theory and SBRF were also used for aerodynamic modeling of the NACA 0012 airfoil. One problem to present is the pitch moment response of the airfoil to a pitching motion at $M=0.764$ for $\alpha=8.5^{\circ} \sin (\omega t)$ with $k=0.1$.
For the identification of the Volterra kernels, Da Ronch et al. [32] used a CFD-generated training maneuver as the data source. The variation of the maneuver angle of attack with time is shown in Fig. 29(a). The Mach number of this maneuver was set to 0.764 similar to the target motion and the reduced frequency was 0.1.

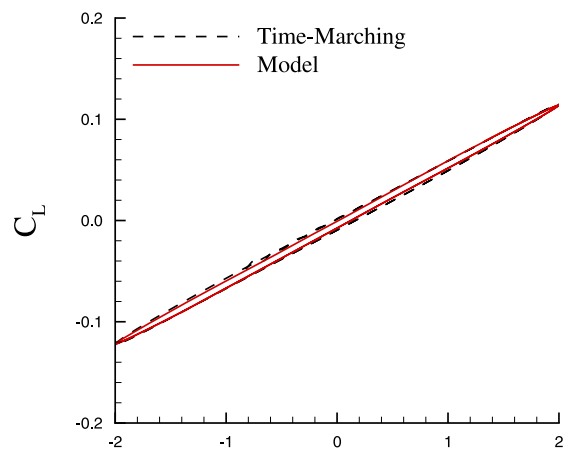

Angle of attack, $\alpha(\mathrm{deg})$

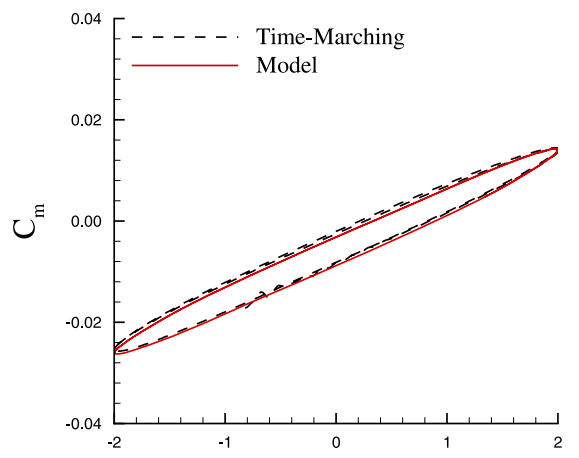

Angle of attack, $\alpha(\mathrm{deg})$

$\alpha_{e}=2^{o} \sin (\omega t), k=0.01, M=0.32$

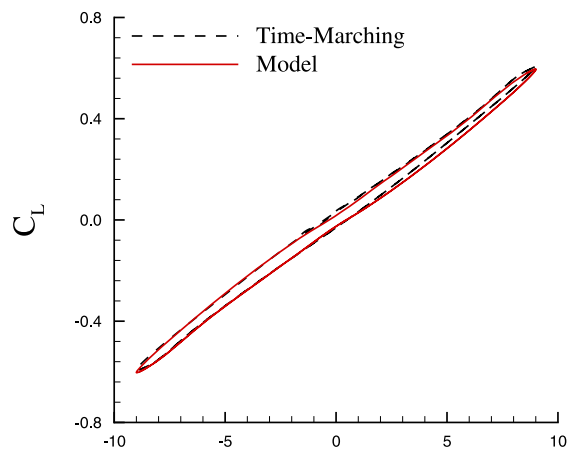

Angle of attack, $\alpha$ (deg)

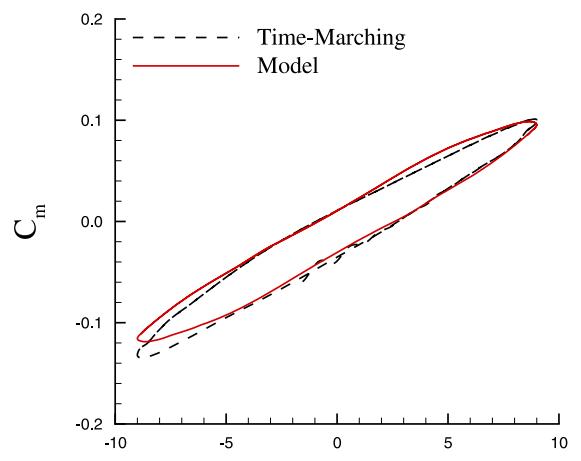

Angle of attack, $\alpha$ (deg)

$\alpha_{e}=9^{\circ} \sin (\omega t), k=0.015, M=0.66$
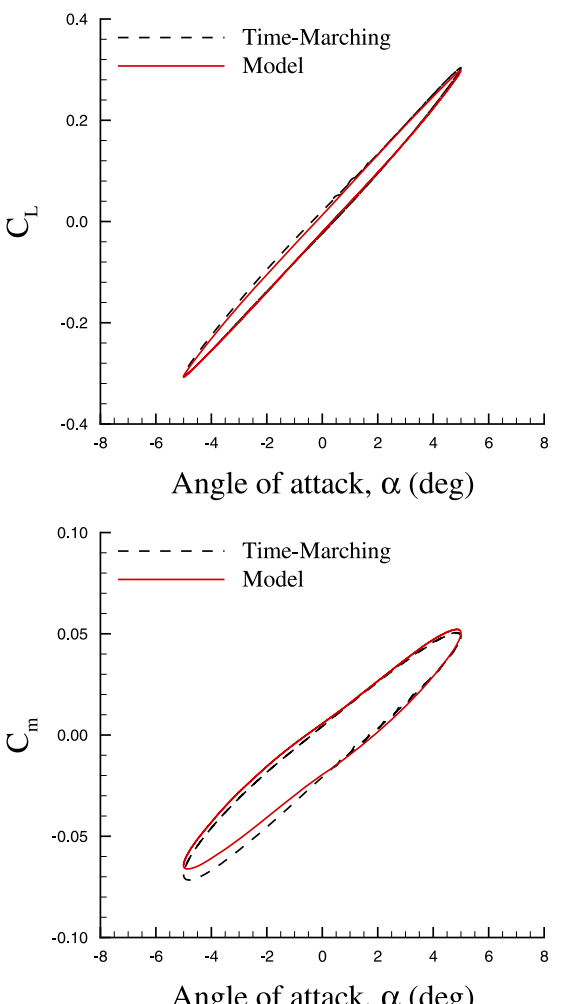

$\alpha_{e}=5^{\circ} \sin (\omega t), k=0.01, M=0.46$

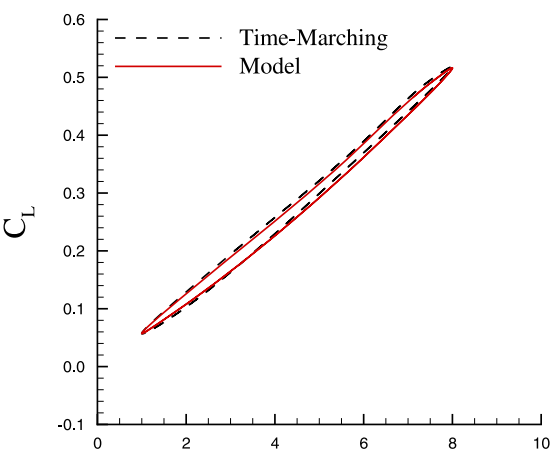

Angle of attack, $\alpha(\mathrm{deg})$

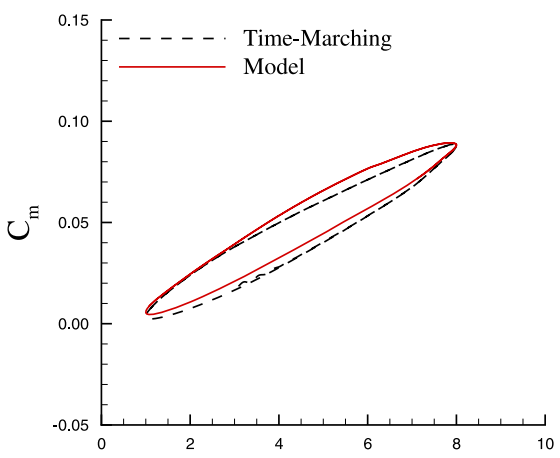

Angle of attack, $\alpha$ (deg)

$\alpha_{e}=4.5^{\circ}+3^{\circ} \sin (\omega t), k=0.035, M=0.46$

Fig. 37. ROM prediction of plunging motions.

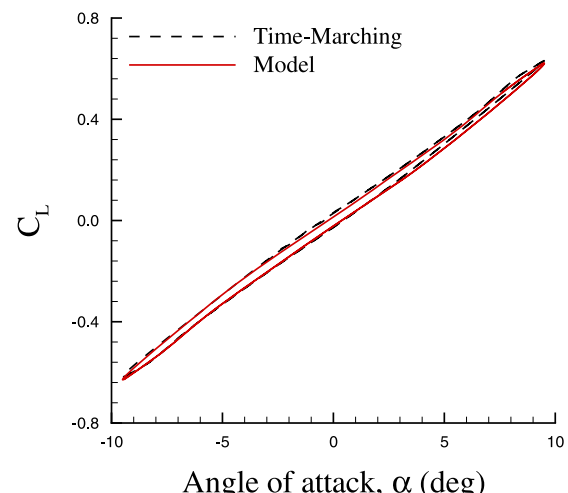

Angle of attack, $\alpha$ (deg)

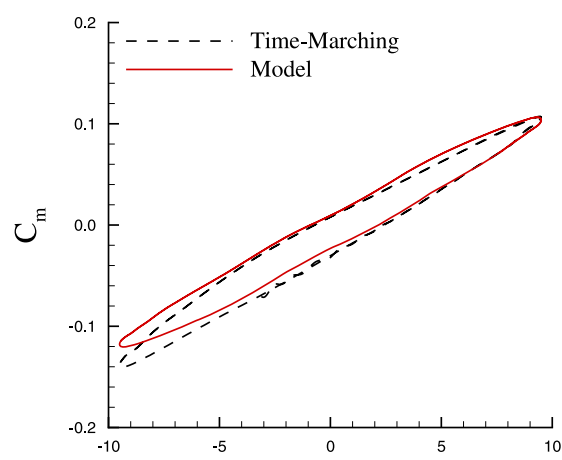

Angle of attack, $\alpha$ (deg)

$\alpha_{e}=9.5^{\circ} \sin (\omega t), k=0.01, M=0.58$

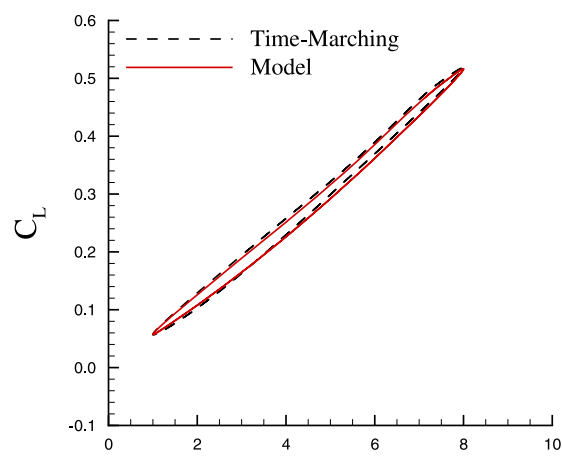

Angle of attack, $\alpha(\mathrm{deg})$

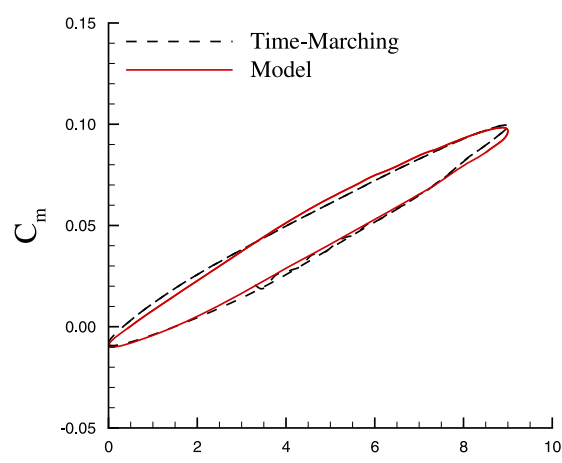

Angle of attack, $\alpha(\mathrm{deg})$

$\alpha_{e}=4.5^{\circ}+3.5^{\circ} \sin (\omega t), k=0.05, M=0.58$ 
The mean angle of attack was zero, but the amplitude changes in time; the maximum angle of attack in the maneuver is $14^{\circ}$ to excite nonlinear aerodynamics due to shock-induced separation. The pitch moment coefficient of this maneuver computed using the RANS grid is illustrated in Fig. 29(b). The Volterra model is also included in the same figure for comparison with the training signal used in the identification process. The overall agreement is reasonable. However, deviations can be observed at both ends of the time interval.
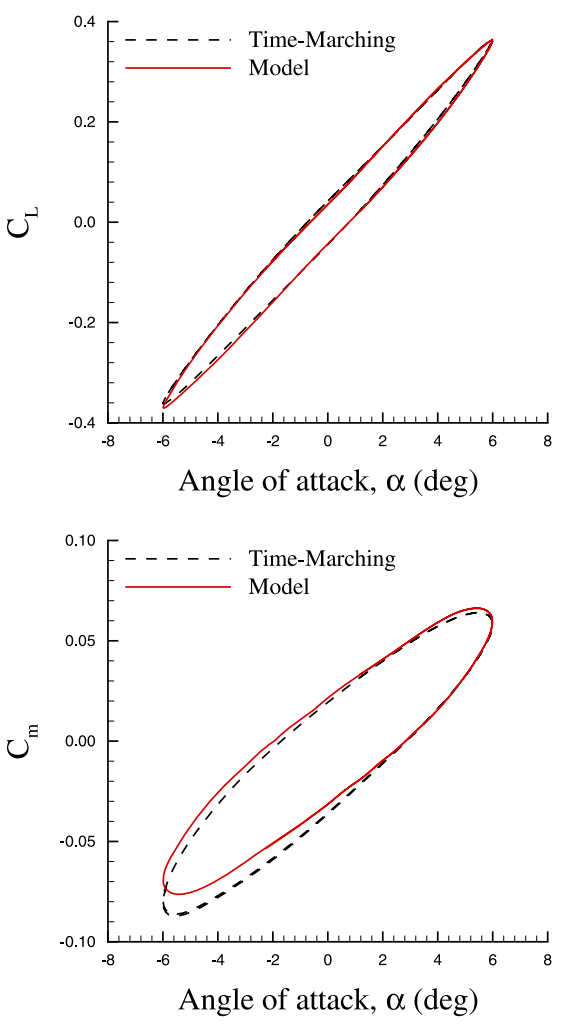

$\alpha=6^{\circ} \sin (\omega t), k=0.02, M=0.32$
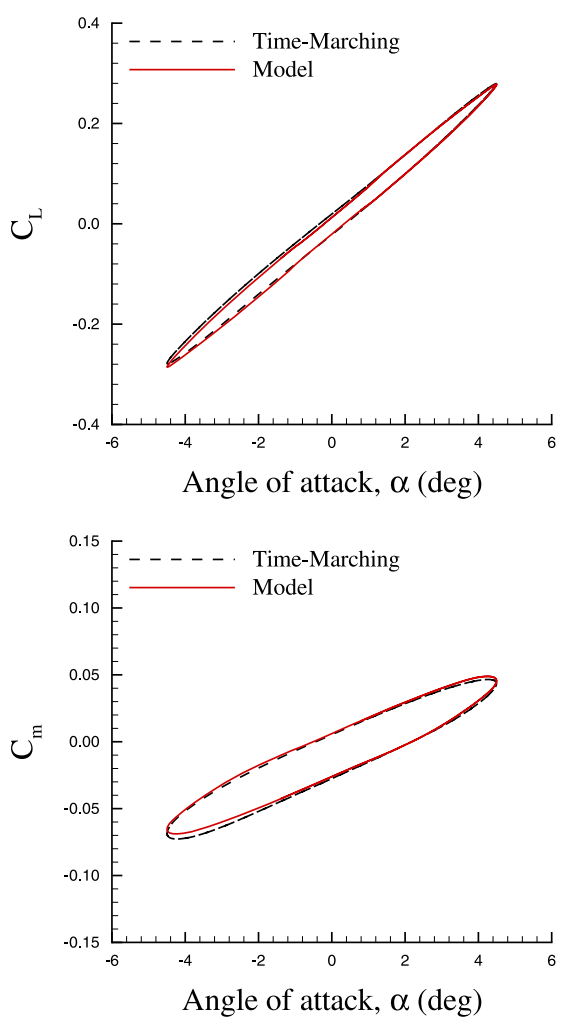

$\alpha=4.5^{\circ} \sin (\omega t), k=0.015, M=0.66$
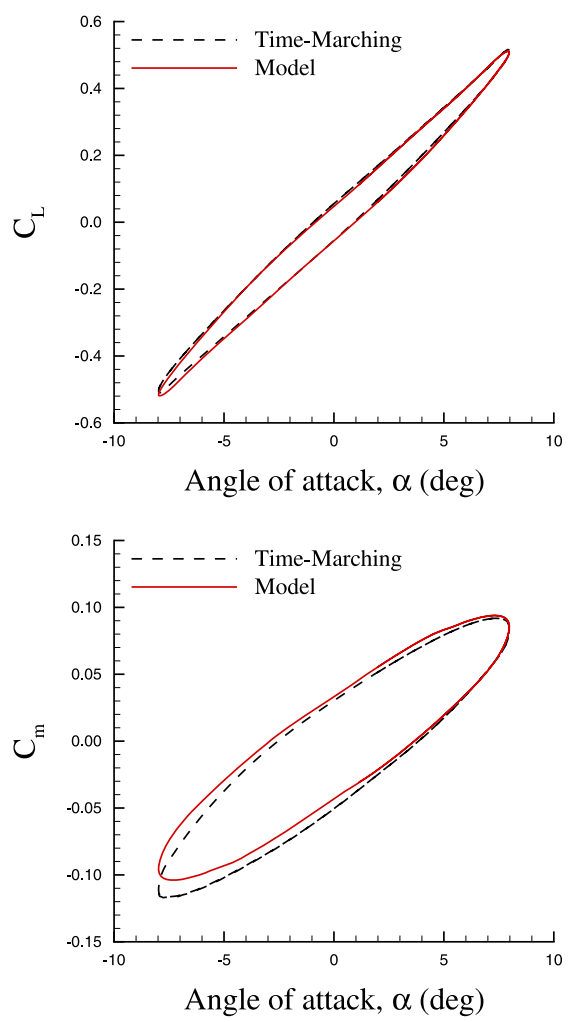

$\alpha=8^{\circ} \sin (\omega t), k=0.025, M=0.46$
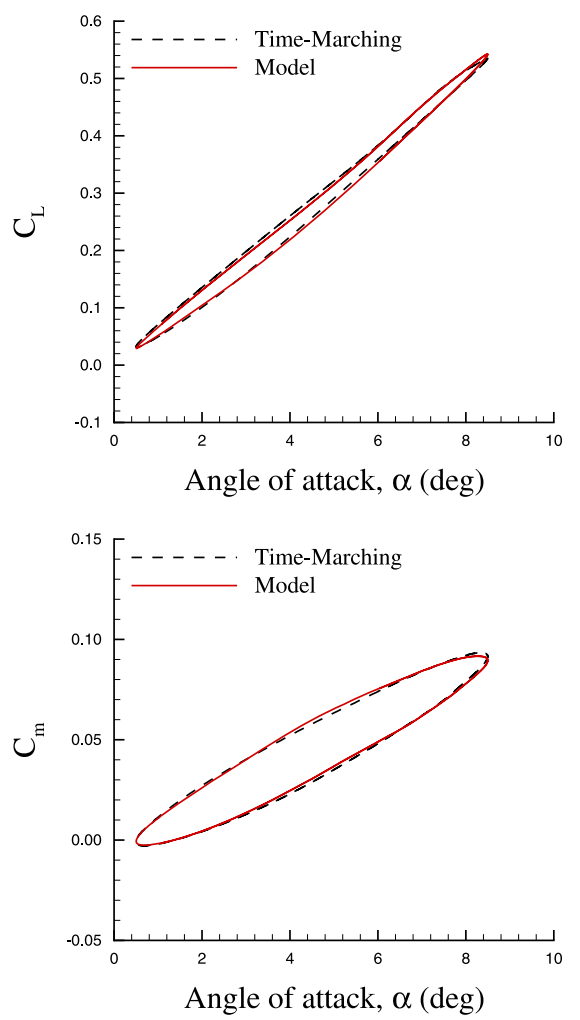

$\alpha=4.5^{\circ}+4^{\circ} \sin (\omega t), k=0.02, M=0.37$
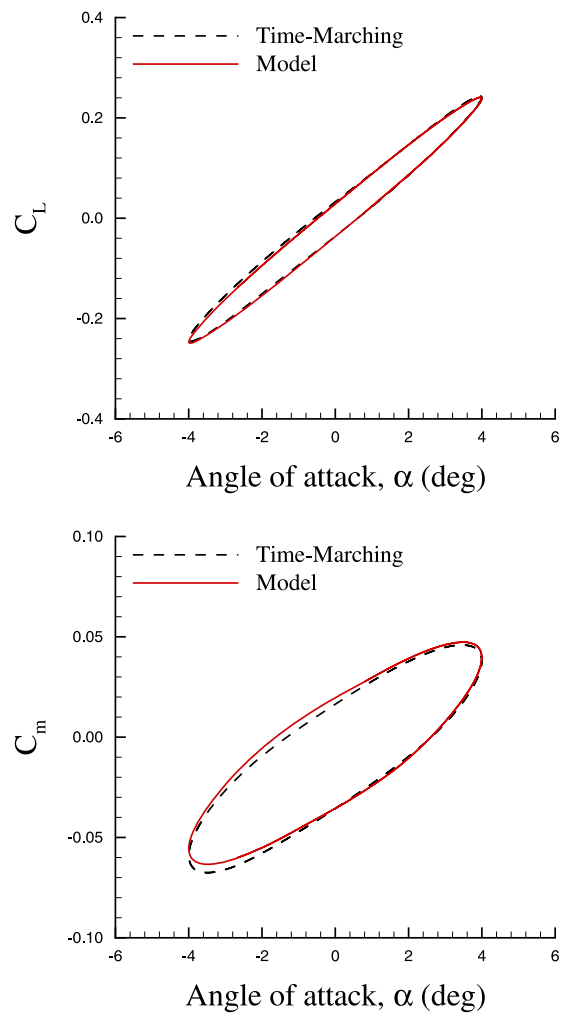

$\alpha=4^{\circ} \sin (\omega t), k=0.05, M=0.58$
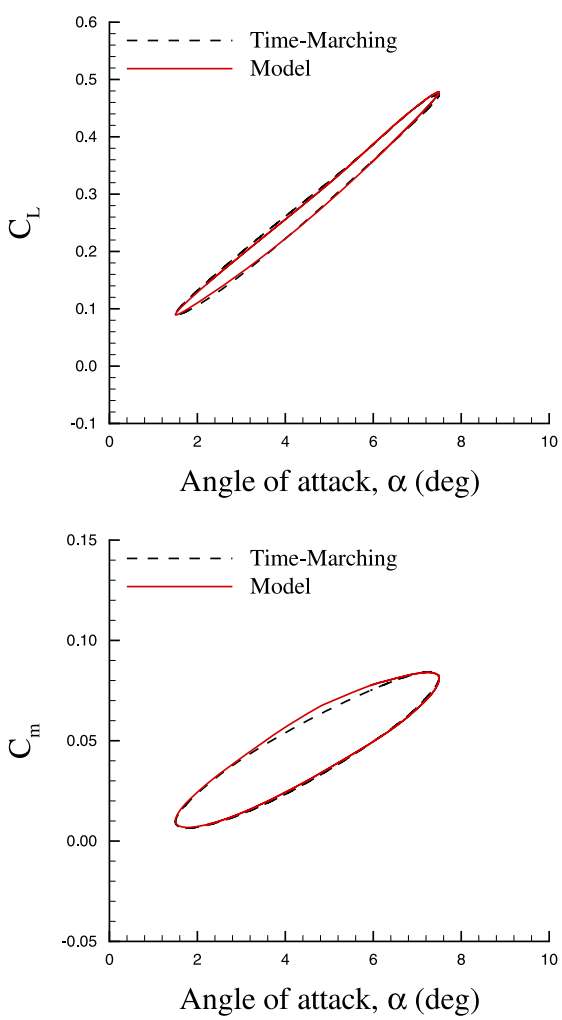

$\alpha=4.5^{\circ}+3^{\circ} \sin (\omega t), k=0.035, M=0.46$

Fig. 38. ROM prediction of pitching motions. 
As the airfoil is symmetric and oscillates around zero degrees mean angle of attack, the resulting aerodynamic loads are odd functions of the angle of attack change. As a result, any odd kernel was neglected in the identification process. The Volterra

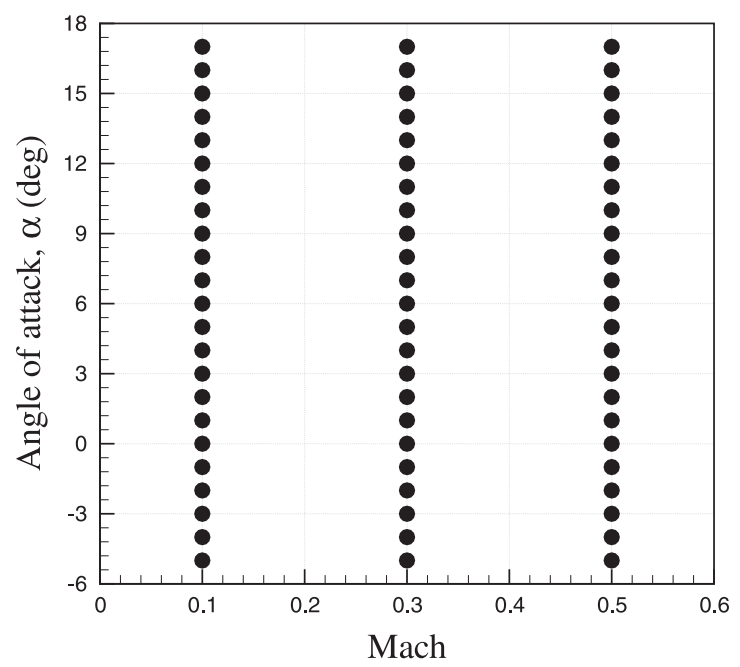

Fig. 39. Design space samples.
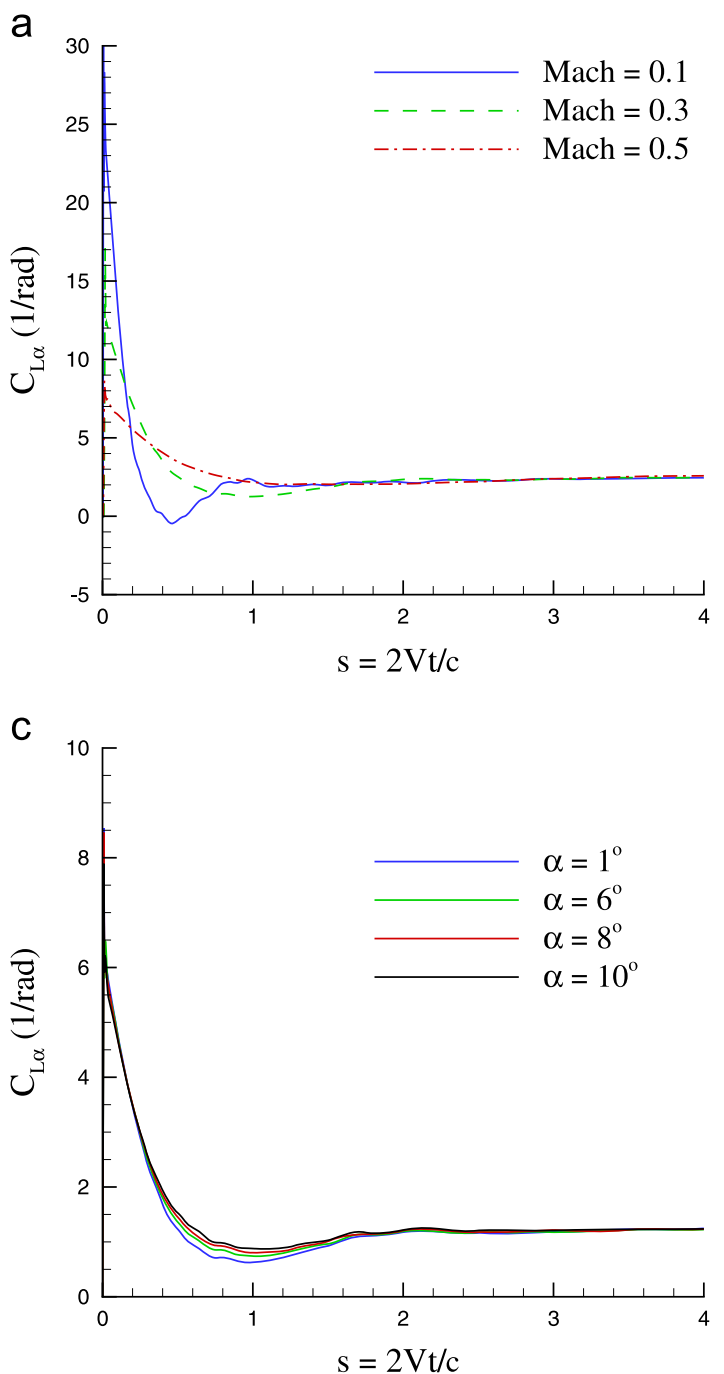

model used in the results includes kernels up to third order as follows:

$$
\begin{aligned}
& H_{1}^{\alpha} \quad H_{1}^{\dot{\alpha}} \quad H_{1}^{\ddot{\alpha}} \\
& H_{2}^{\alpha, \dot{\alpha}} \quad H_{2}^{\dot{\alpha}, \ddot{\alpha}} \\
& H_{3}^{\alpha, \dot{\alpha}, \dot{\alpha}} \quad H_{3}^{\dot{\alpha}, \dot{\alpha}, \ddot{\alpha}} \quad H_{3}^{\alpha, \alpha, \ddot{\alpha}}
\end{aligned}
$$

The upper bound of the integer $k$ in Eq. (37), which indicates the number of previous time-steps to account for, was set to one for the terms $H_{1}^{\alpha}$ and $H_{1}^{\dot{\alpha}}$; for the other kernels, it was set to zero. The effect of including more terms in the model was assessed during the identification of the Volterra model, and in the subsequent comparison for the target maneuver.

Fig. 30 conveys the unsteady pitch moment coefficient for the target maneuver. The CFD solution exhibits nonlinear characteristics at the higher angles of attack. The Volterra model including the kernels shown in Eq. (46) is illustrated in the same figure for comparison. Including first-order kernels, the error norm in the prediction of the target maneuver was $6.78 \%$. Second-order kernels were then added and the Volterra model deviated by $6.87 \%$ from the CFD solution. By introducing third-order kernels (this model is shown in Fig. 30), the error norm slightly drops to 6.18\%. Despite using kernels up to third order, the agreement is not excellent. The overall fit of the model is reasonable, but there is no sign of the prediction of any non-linear features.

b
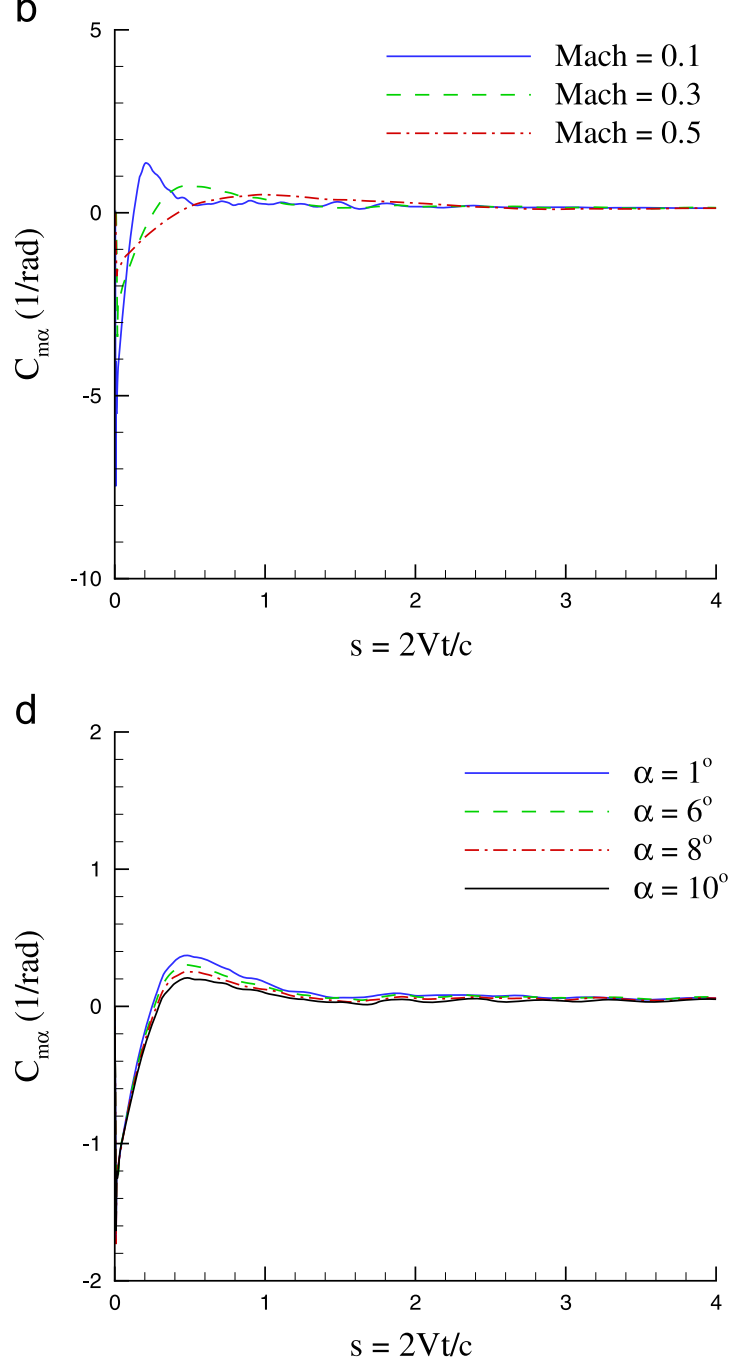

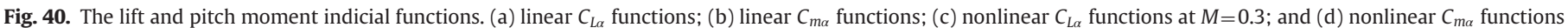
at $M=0.3$. 
The mediocre performance of the Volterra model may be attributable to two aspects. To obtain a robust predictive model, in combination with kernels of the second and third order, the influence of the previous time-history on the current time instant was limited. Second, the training case considered may not be optimal for the model identification.

An SBRF model was also constructed to predict the pitch moment response of similar target maneuvers. This model was created from CFD solutions corresponding to 30 different combinations of $\alpha_{A}$, constrained to vary between $0^{\circ}$ and $10^{\circ}$. With an increment in $\alpha_{A}$ of $0.25^{\circ}$, the parameter space was initially covered for $75 \%$ of all the possible combinations. Fig. 31 depicts the carpet plot of the pitch moment coefficient for each value of the parameter $\alpha_{A}$ obtained using the SBRF model. The axis "Iteration" indicates the time evolution through the last cycle of the simulations.

Next, the number of initial training CFD solutions was iteratively reduced to focus on the predictive capabilities of the model.
Four CFD solutions were eventually retained, and these are included as large spheres in Fig. 31. Note that two training cases are located at the borders of the parameter space to avoid extrapolation. The remaining two cases were automatically sampled by the algorithm described in reference [117]. Note also that there is no close proximity of the four training cases to the amplitude of $8.5^{\circ}$ used in the target maneuver.

The ROM predictions are evaluated for the target maneuver with a Mach number of 0.764 . The comparisons are presented in Fig. 32 which shows the reduced-order model closely approximates the reference solution (the error norm is $1.7 \%$ ), featuring very similar nonlinear characteristics at higher angles as well. Note that this model can only predict responses to motion at a constant reduced frequency of $k=0.1$. The number of training maneuvers, and therefore the computational cost of this model, will significantly increase for aerodynamic modeling throughout the angle of attack/Mach number/frequency space. a

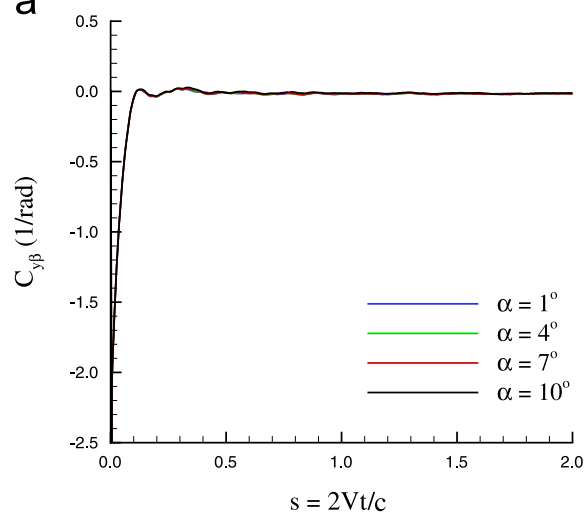

d

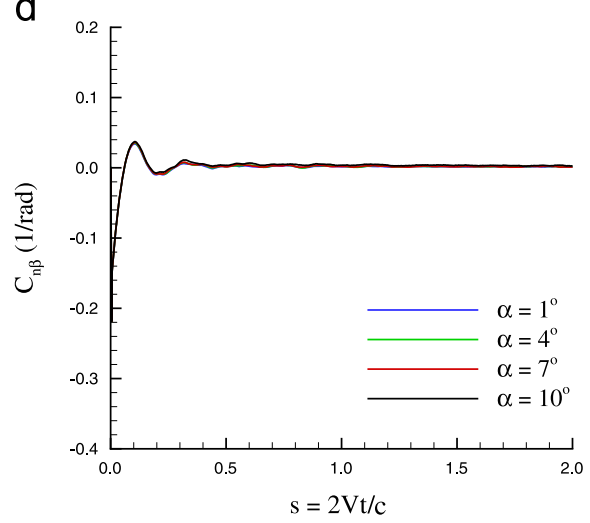

9

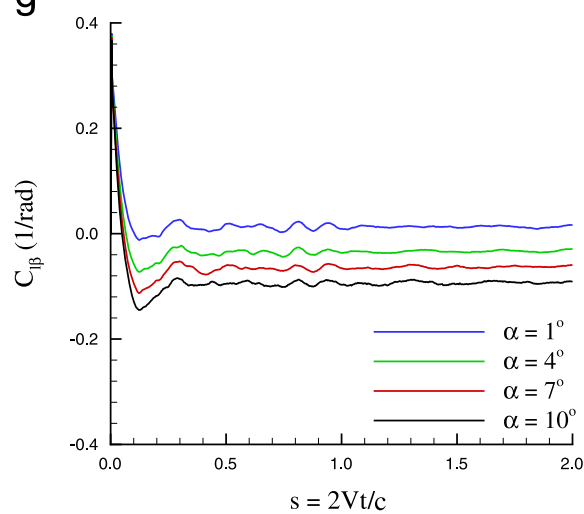

b

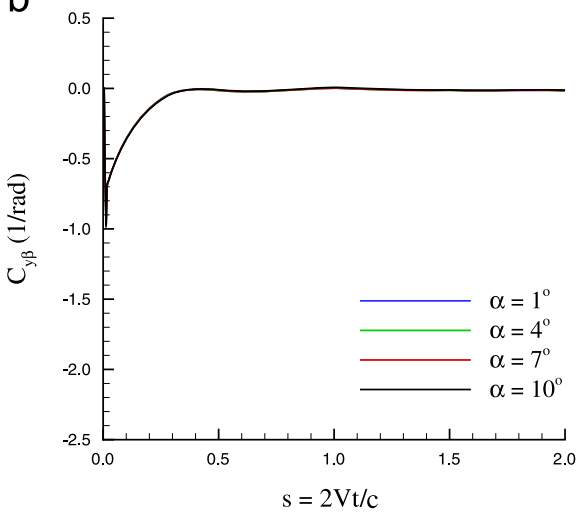

e

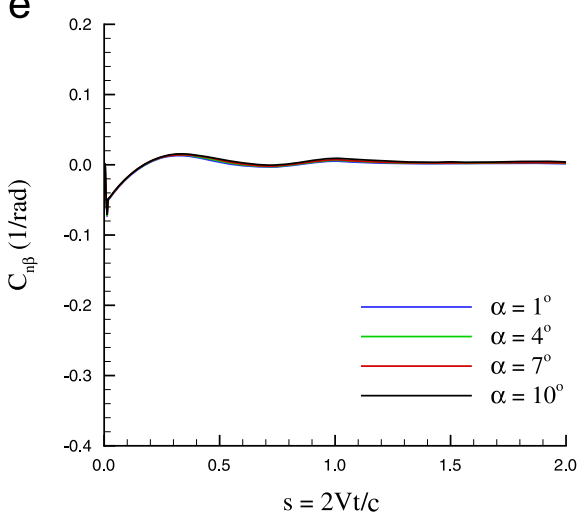

h

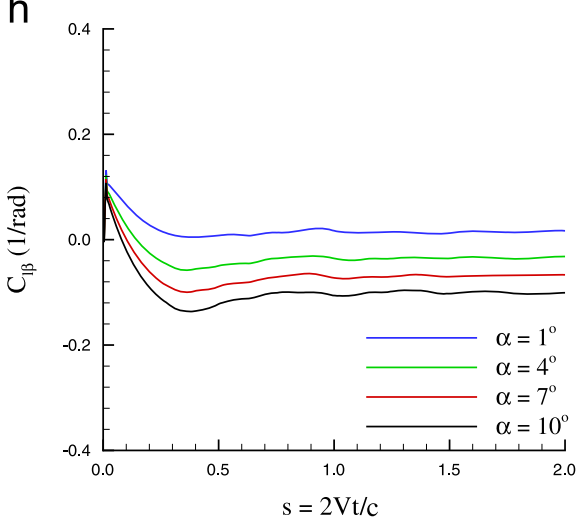

C

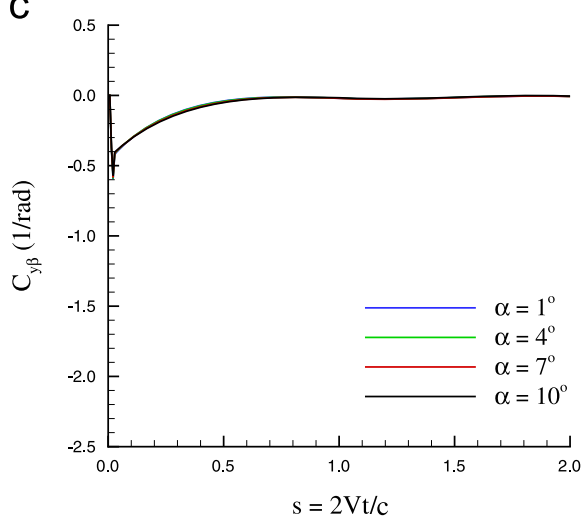

f

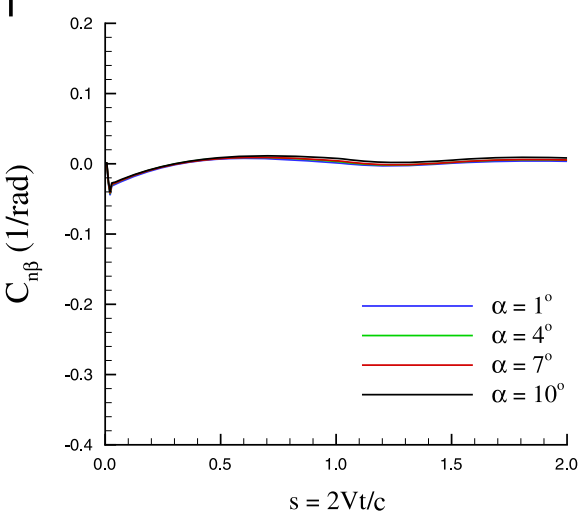

i

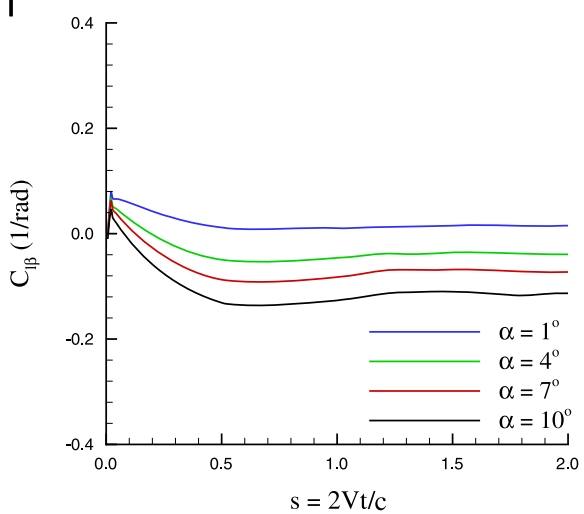

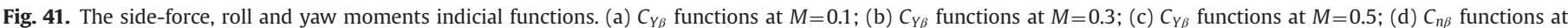
$M=0.1$; (e) $C_{n \beta}$ functions at $M=0.3$; (f) $C_{n \beta}$ functions at $M=0.5$; (g) $C_{l \beta}$ functions at $M=0.1$; (h) $C_{l \beta}$ functions at $M=0.3$; and (i) $C_{l \beta}$ functions at $M=0.5$. 


\subsection{SDM aerodynamics modeling}

A ROM based on an indicial response function, along with a timedependent surrogate approach, is reviewed for the SDM aerodynamic modeling in the angle of attack/Mach number/frequency space.

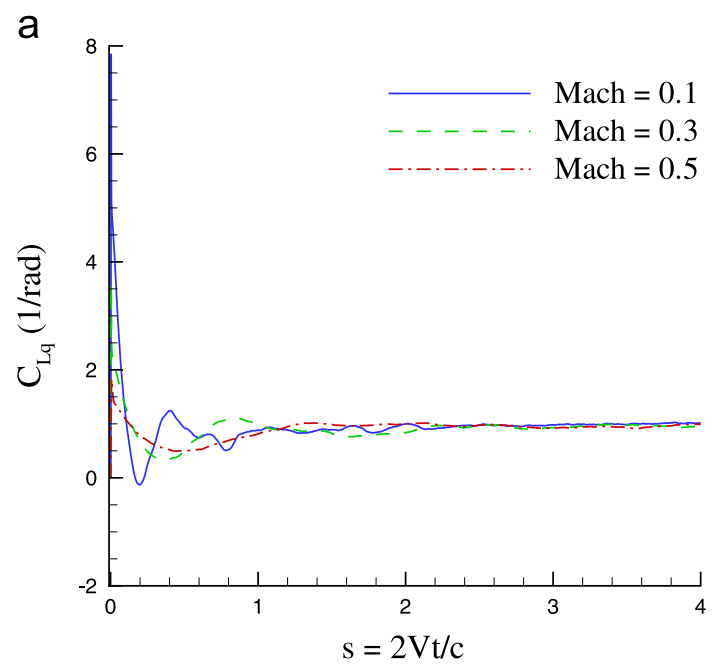

Fig. 42. The lift and pitch moment indicial functions with a unit step change of normalized pitch rate at different Mach numbers.

a

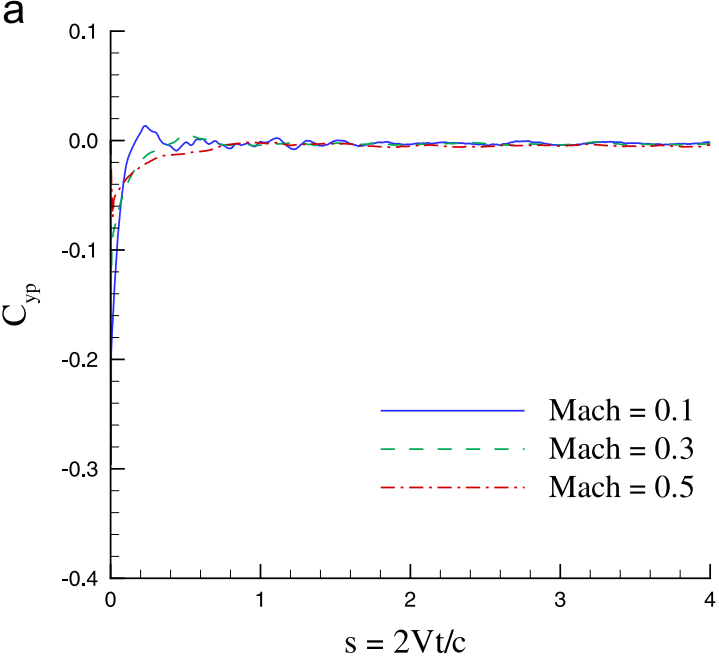

C

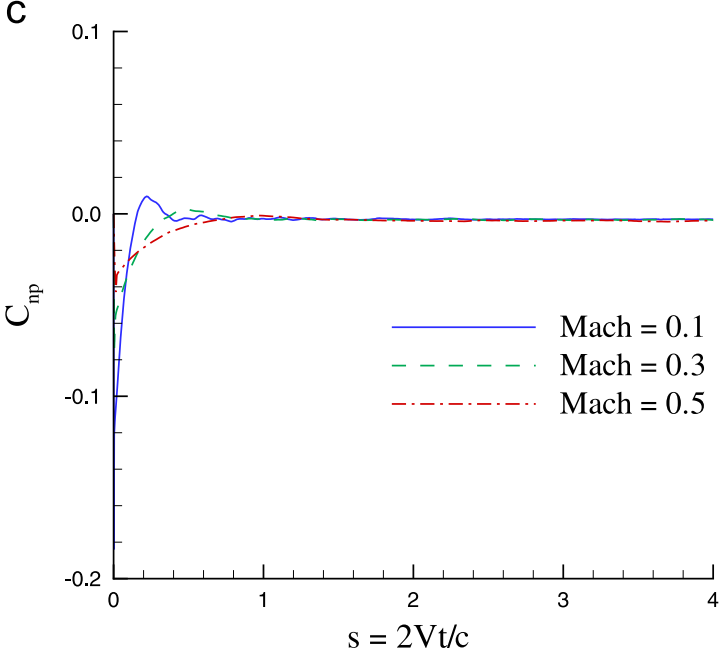

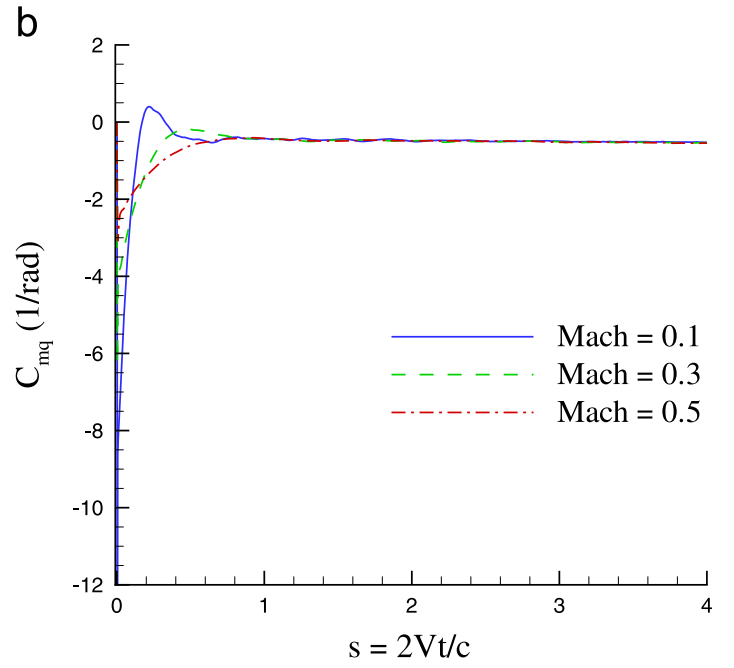

The indicial response functions of the SDM aircraft are interpolated from some available samples in the angle-of-attack and free-stream Mach number space. Note that these functions only need to have dependency on angle-of-attack and Mach number, and once they have been calculated they could be used to predict

b

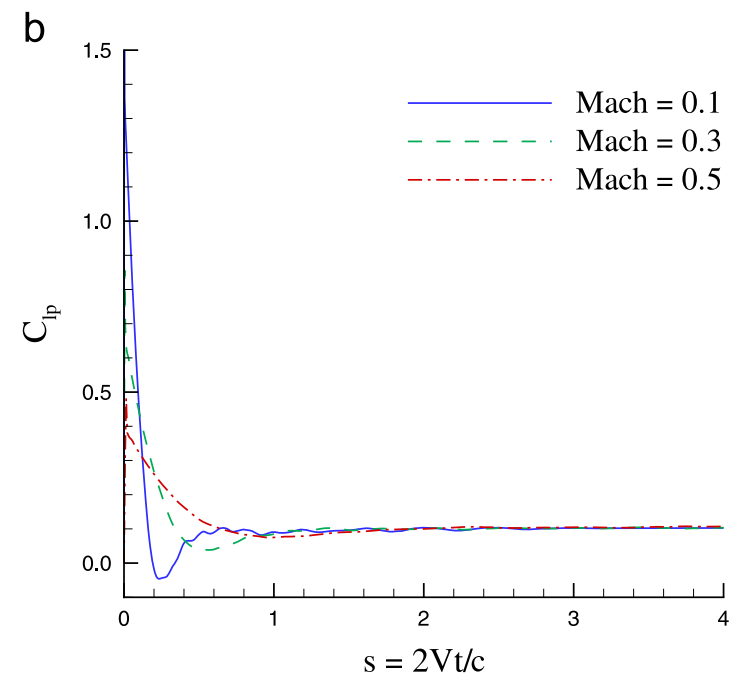

Fig. 43. The lateral coefficients with a unit step change in the normalized roll rate. 
the aerodynamic response to any frequency of interest. For example the samples could be generated using the methods of factorial designs, Latin hypercube sampling, low discrepancy sequences, or designs based on statistical optimality criteria (A-, D- and G-optimal designs) [160]. Factorial designs are extremely simple to construct and have been used in this work. The SDM motions considered encompass $\alpha$ and $M$ values in the range of $\left[-10^{\circ}, 10^{\circ}\right]$ and $[0.3,0.7]$, respectively. Assuming symmetrical flow solutions with respect to the angle of attack, the indicial functions are only calculated for positive angles of attack.

A set of samples including 50 points is defined on the $\alpha$ and $M$ space using factorial design; these points are shown in Fig. 33. The indicial functions are calculated using CFD with the grid motion approach for each sample condition. All these calculations started from a steady-state solution such that the Mach number in the steady-state simulations corresponds to each sample Mach number. The step function calculations are second order in time with a non-dimensional time step of $\Delta t^{*}=\Delta t . V / c=0.01$. The calculated indicial functions due to angle of attack are shown in Fig. 34 for $M=0.3$ and $M=0.6$. Fig. 34(a) and (b) shows that the indicial lift has a peak at $s=0$ followed by a rapidly falling trend. The lift again builds up and asymptotically reaches the steady-state value. The pitch moment also has a negative peak at $s=0$ as shown in Fig. 34 (c) and (d). Fig. 34 shows that the initial values of indicial functions are invariant with angle of attack, but the intermediate trend and steady-state values change, depending on the angle of attack.
Although, the final values of indicial lift are nearly unchanged for angles of attack below $5^{\circ}$, but the pitch moment final values are different even at small angles of attack due to vortices on the wing.

The effects of Mach number on the lift and pitch moment indicial functions with respect to pitch rate are shown in Fig. 35. Fig. 35 shows that increasing Mach number results in the increase of steady-state $C_{L q}$ and the decrease of steady-state $C_{m q}$ (the so-called pitch damping derivative). Fig. 36 compares the steadystate $C_{m q}$ values calculated from the CFD code with the out-ofphase components of pitch moment derivative, i.e. $C_{m q}+C_{m \dot{\alpha}}$ measured at different Mach numbers and zero degrees angle of attack; these experimental data are detailed by Da Ronch et al. [161]. Like the static predictions, the CFD values slightly underestimate the experimental pitch moment data, although the CFD predictions do not include the effects of the rate of change of angle of attack, i.e. $C_{m \dot{\alpha}}$. $C_{m q}$ is the largest factor in the sum $C_{m q}+C_{m \dot{\alpha}}$, typically accounting for $90 \%$ of the sum. Again, the underestimation of experimental data is likely due to different inlet geometries in the wind-tunnel and the SDM geometry used in this work. Note that the indicial function approach allows the direct calculation of pitch damping derivatives but extraction of dynamic derivatives from harmonic motions results in the in-phase and out-of-phase components [161] and additional work required to separate each derivative from these components.
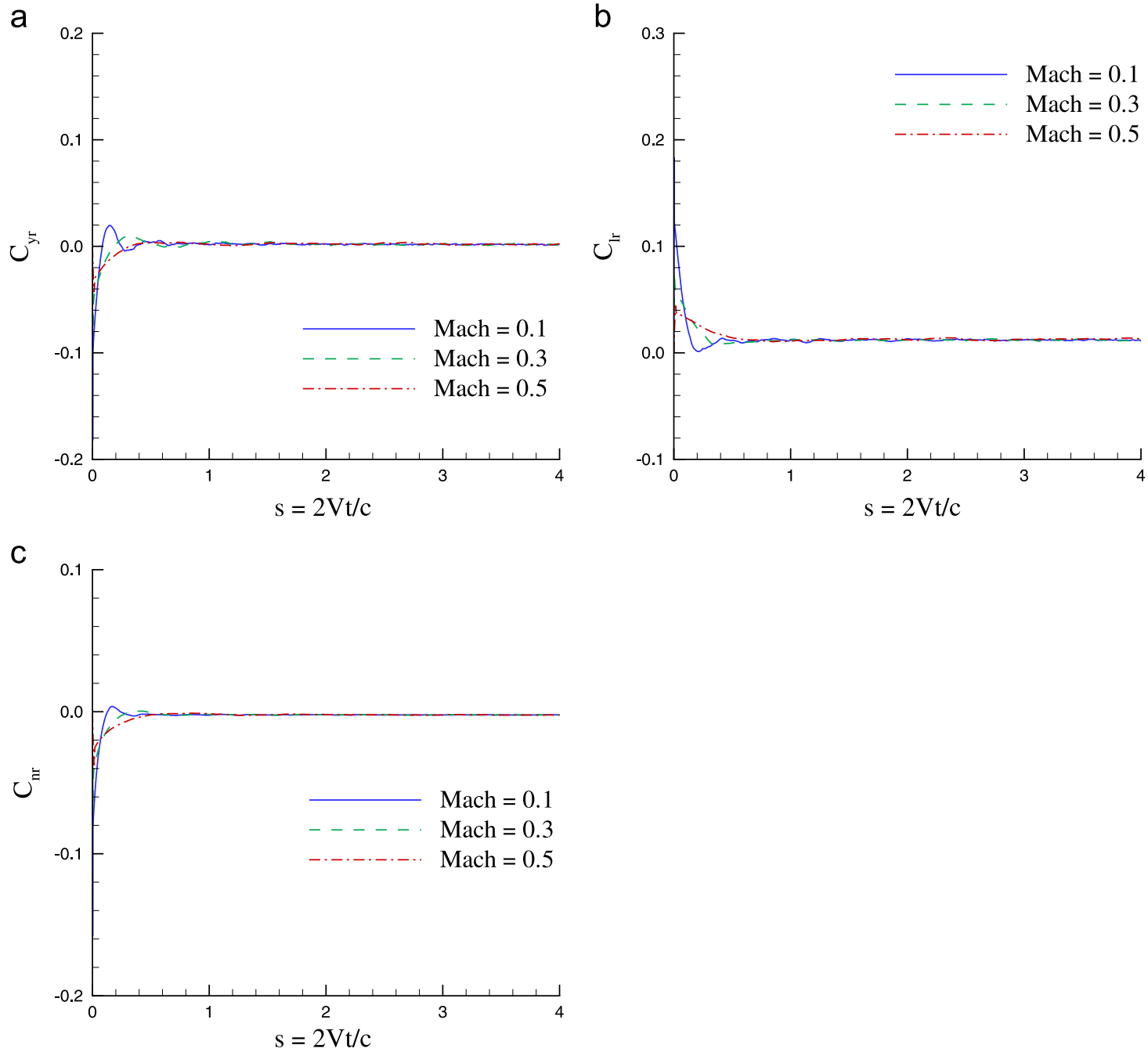

Fig. 44. The lateral coefficients with a unit step change in the normalized yaw rate. 
A nonlinear ROM based on indicial response theory is created along with a time-dependent surrogate model to determine the terms in Eq. (24) at each time step. The validity of the ROM is tested for several arbitrary pitching and plunging motions. These motions start from different steady-state conditions (not being used during ROM creation) and run for different amplitudes and frequencies. The ROM predictions are compared with timeaccurate CFD simulations in Figs. 37 and 38. Figs. 37 and 38 show that the ROM lift and pitch moment predictions agree well with the time-marching simulation values. Small discrepancies are found in the pitch moment predictions at negative angles of attack. This is likely due to the fact that SDM pitch moment is not symmetric with angle of attack and hence the response

a

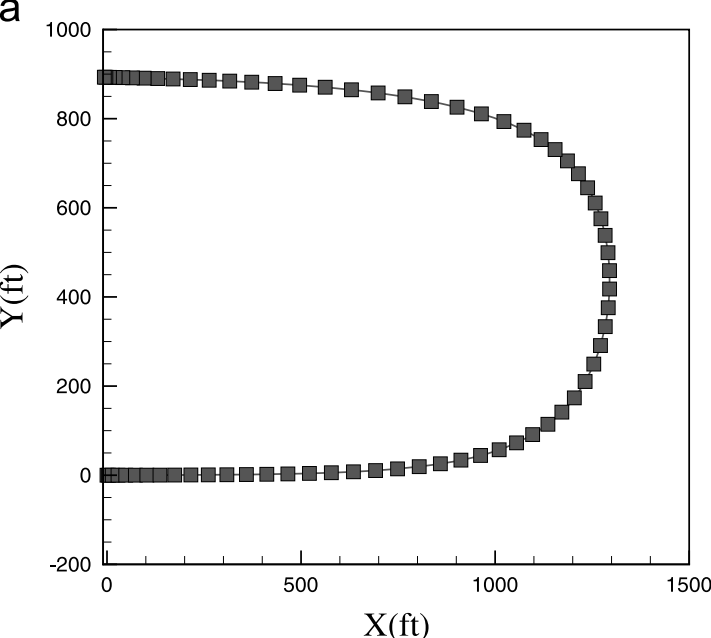

C

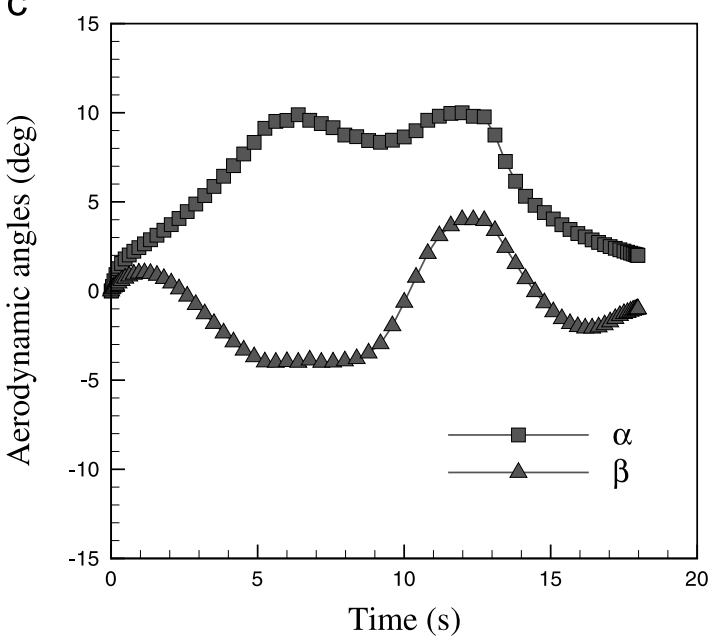

functions generated at positive angles cannot predict the slope changes correctly. Note that the cost of ROM creation and the average cost of generating each time-marching simulation are around 72,500 and $5280 \mathrm{CPU}$ hours using 128 processors $(2.7 \mathrm{GHz})$, respectively. However, the model cost is upfront and once the model is created it can rapidly predict the aerodynamic responses of many motions, all defined within the input space range.

\subsection{SACCON aerodynamics modeling}

Most previous reduced order aerodynamic modeling efforts have been limited to longitudinal motions with the angle of attack
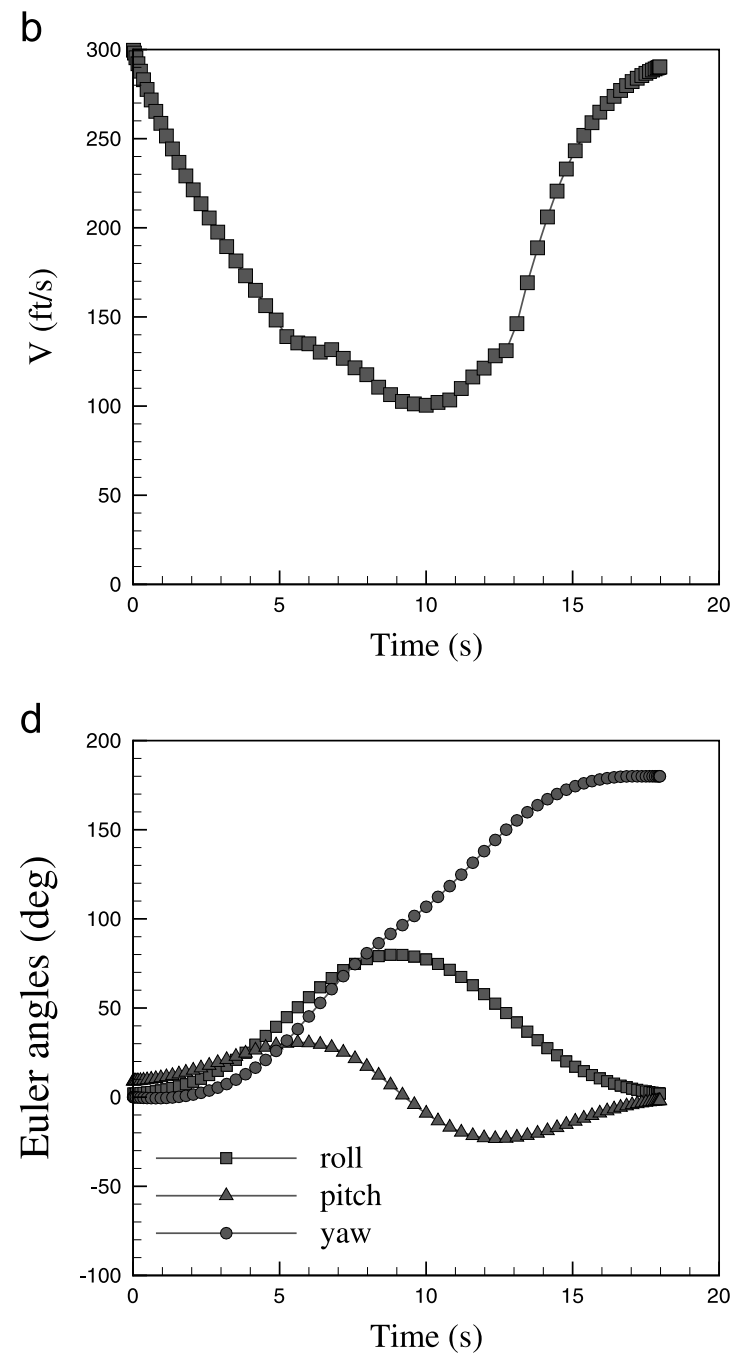

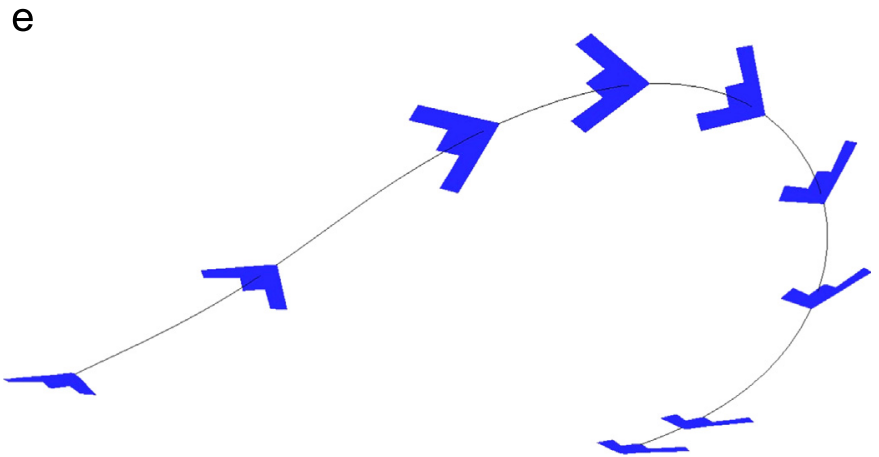

Fig. 45. Half lazy-8 maneuver. (a) ground trajectory; (b) flight velocity; (c) aero angles; and (d) Euler angles; and (e) flight trajectory. 
and pitch rate as input variables. Recently, models based on indicial functions have been used for aerodynamic prediction of both longitudinal and lateral forces and moments [112]. Here, these models are reviewed for prediction of the SACCON aero- dynamic forces/moments during 6DoF maneuvers in the subsonic speed range.

An optimal control approach [162,163] was used to generate 6 DoF maneuvers for the SACCON with the feasible solutions based

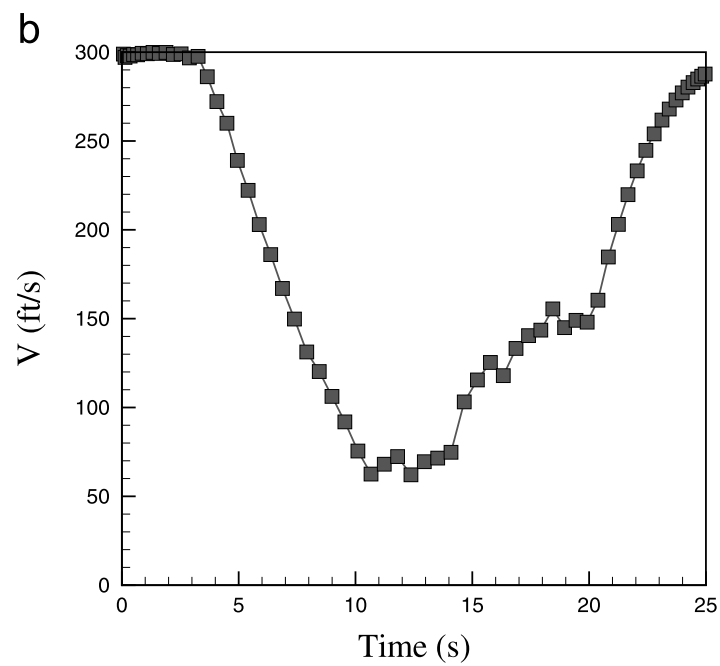

d

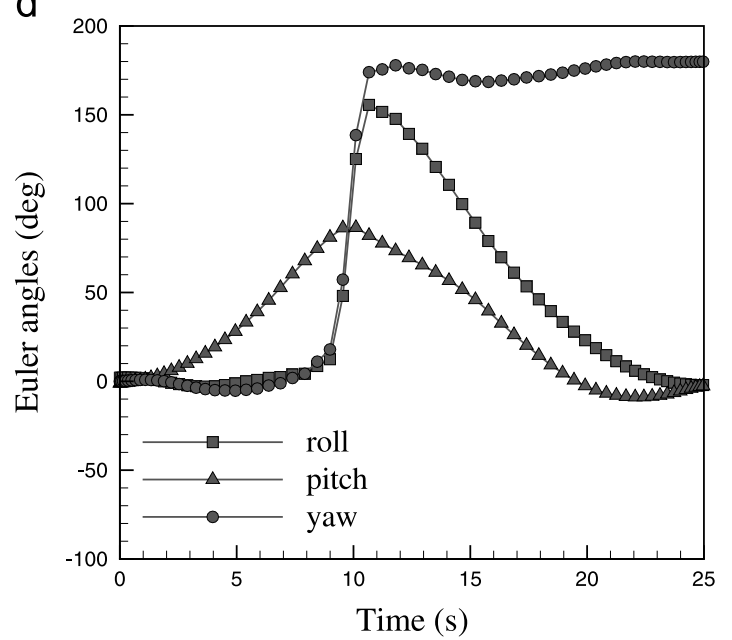

b

d
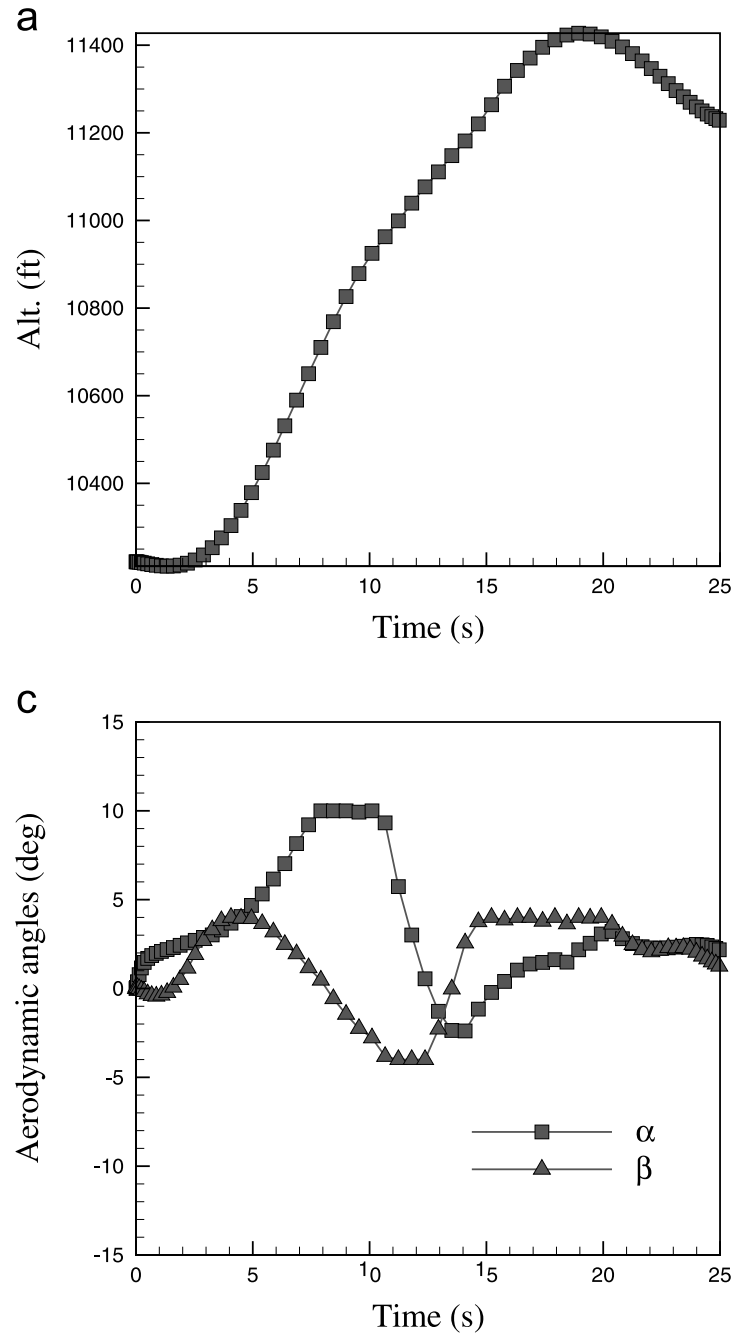

e

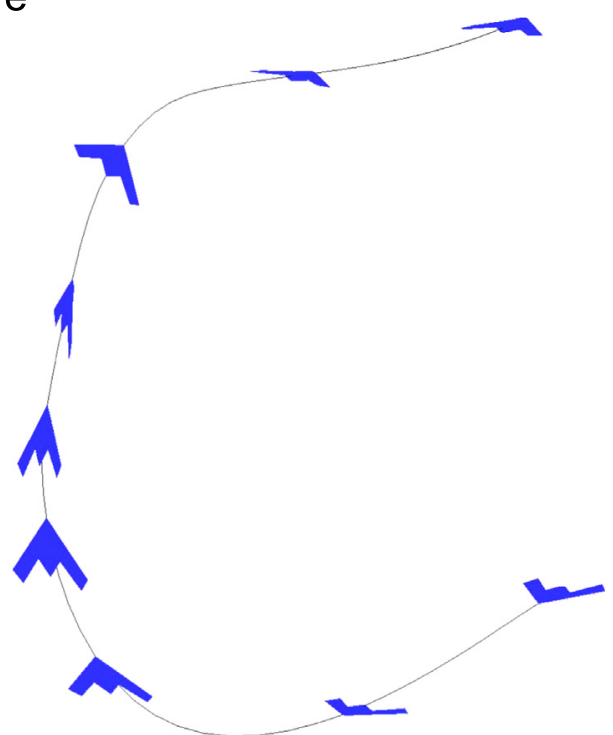

Fig. 46. Immelmann turn maneuver. (a) flight altitude; (b) flight velocity; (c) aero angles; (d) Euler angles; and (e) flight trajectory. 
on the vehicle control and state constraints. The input angle of attack of maneuvers is in the range of $-10^{\circ}$ to $10^{\circ}$ with a Mach number range of $0.1-0.5$. The side-slip angle also ranges from $-5^{\circ}$ to $5^{\circ}$. The side-force, yaw and roll moments are nearly linear with

a

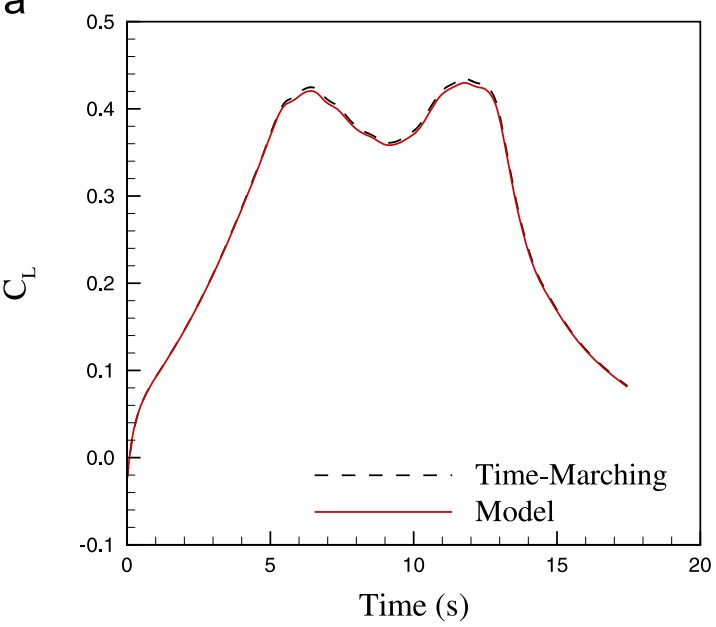

C

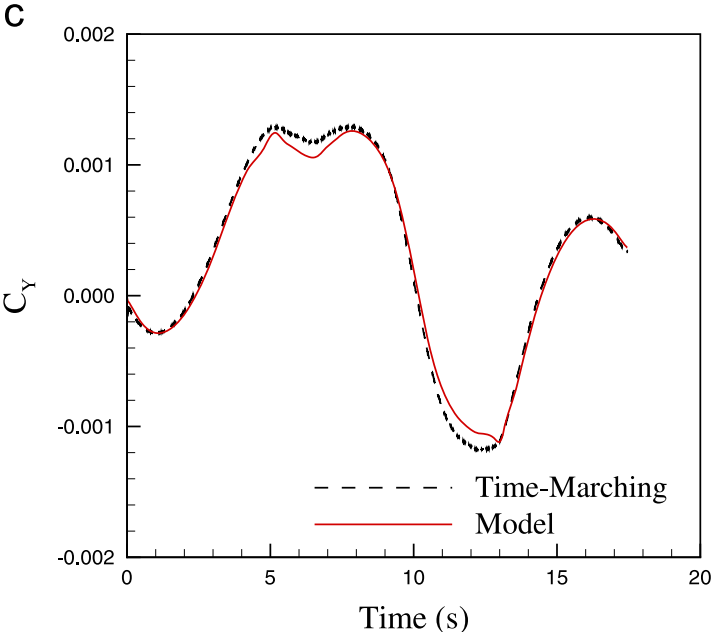

side-slip for small values of side-slip and angles of attack below $15^{\circ}$ as shown in Fig. 13. However, the generation of response functions in the defined input space using CFD is still expensive and makes the creation of a ROM very time consuming. A time-

b

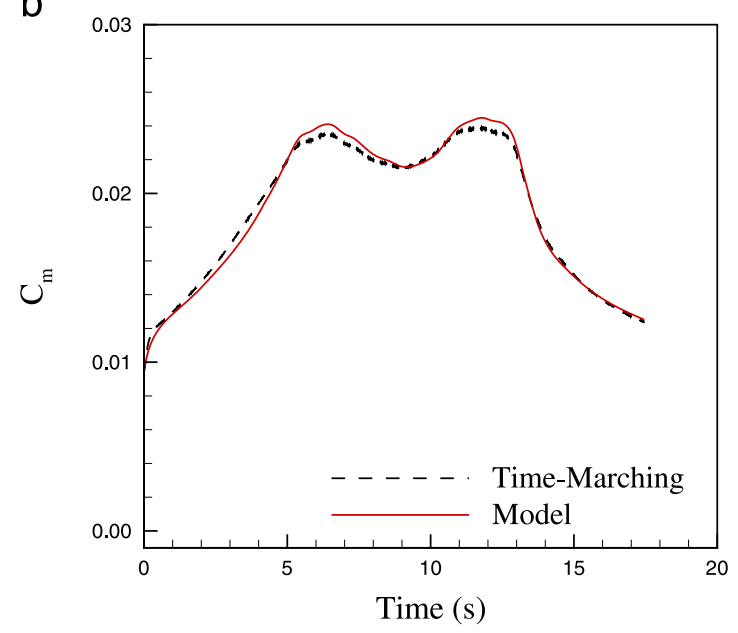

d

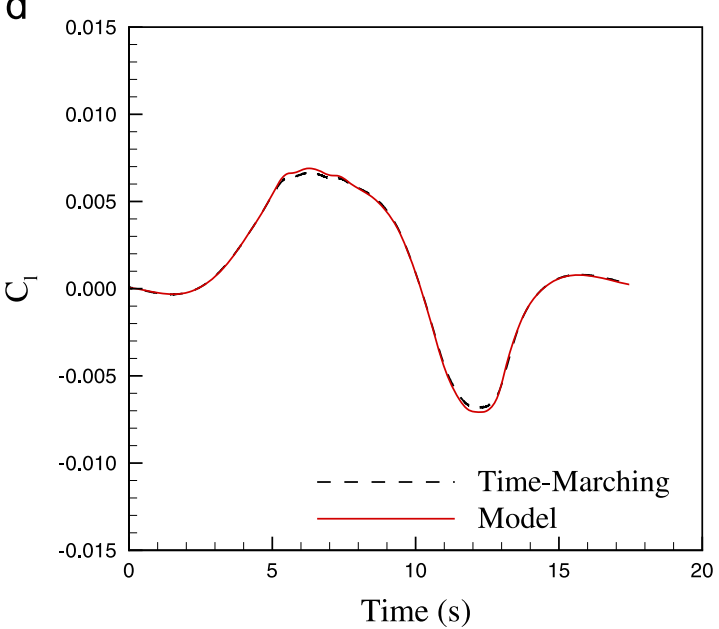

e

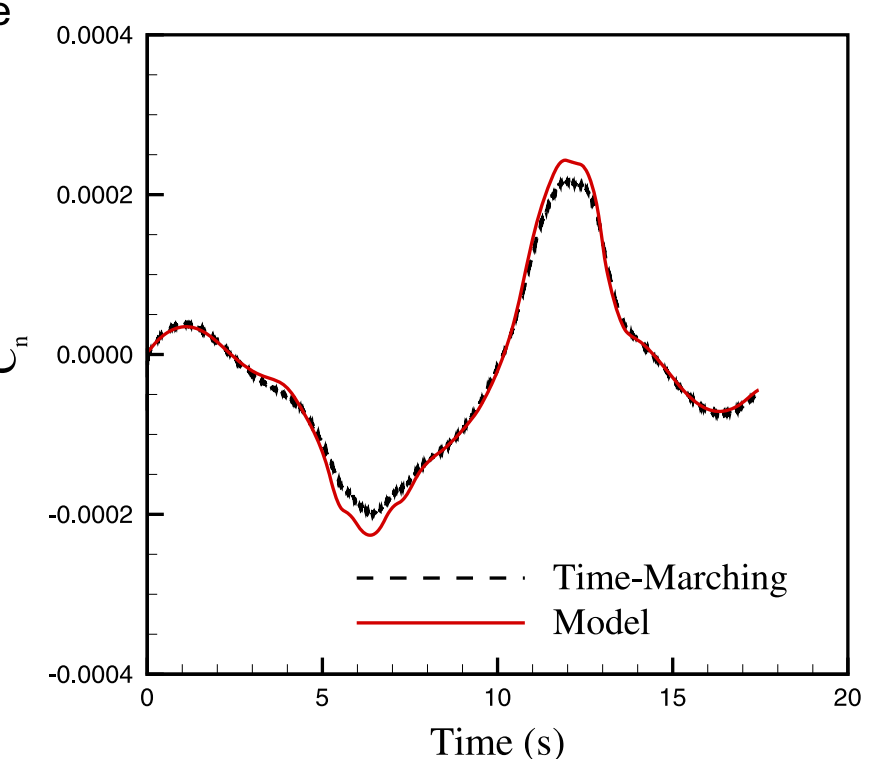

Fig. 47. Aerodynamic modeling of half lazy-8 maneuver. (a) lift coefficient; (b) pitch moment coefficient; (c) side force coefficient; (d) roll moment coefficient; and (e) yaw moment coefficient. 
dependent surrogate model is presented to model SACCON response functions for a new flight condition from some available (observed) responses.

For surrogate modeling of response functions, a set of samples including 33 points is defined on the $\alpha$ and $M$ space using full factorial design. Also, it is assumed that the aerodynamic forces and moments are symmetric about zero degrees angle of attack and hence only the angles between zero and ten degrees are considered for response function calculations. This assumption is not entirely correct but it aids in reducing the computational cost of generating response functions. The sample points are uniformly spaced over positive $\alpha$ values for Mach numbers of 0.1, 0.3, and 0.5 and are shown in Fig. 39.
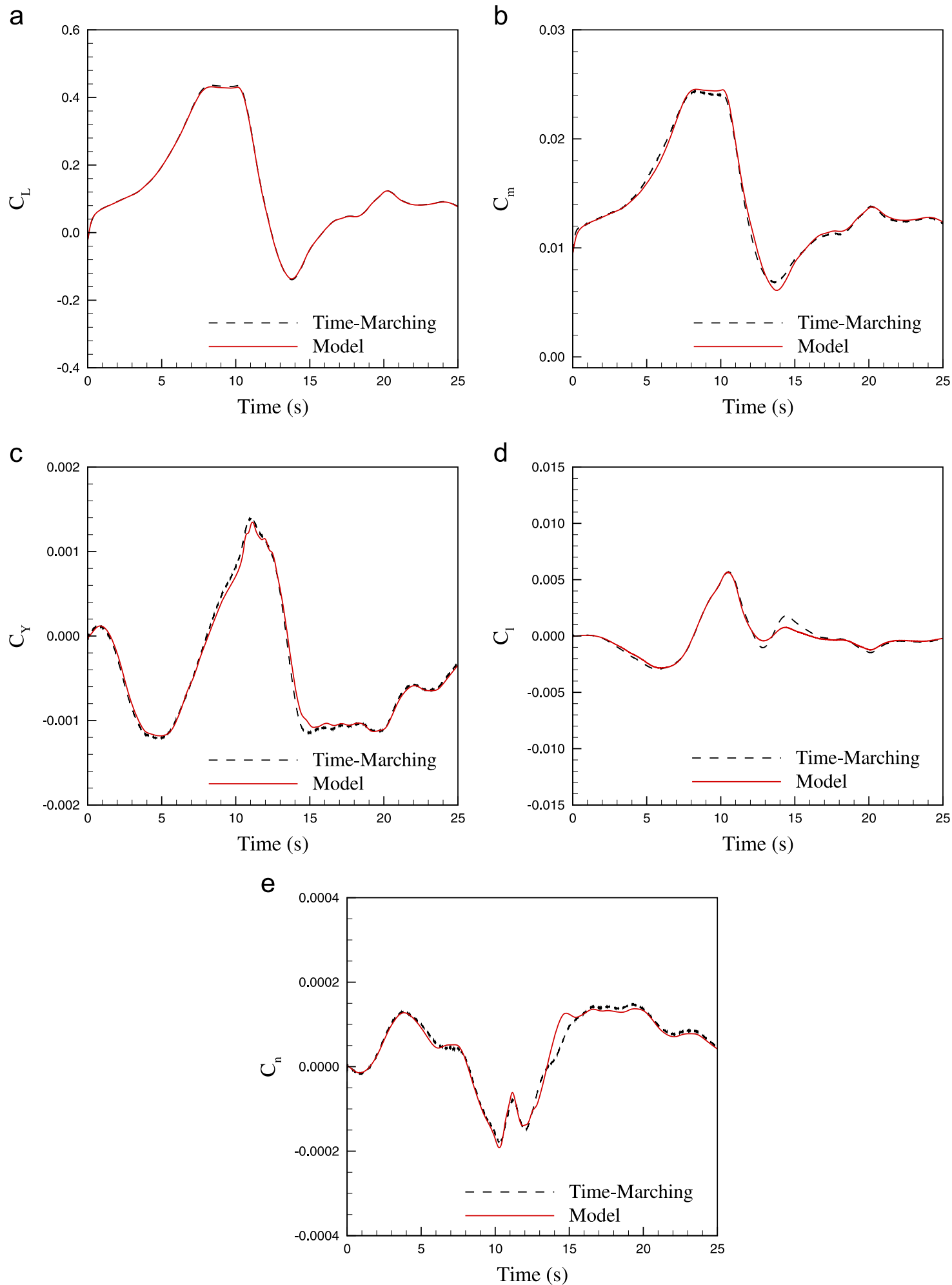

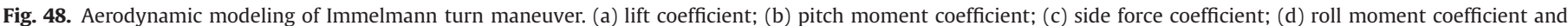
(e) yaw moment coefficient. 
All response computations started from a steady-state solution and then advanced in time using second-order temporal accuracy. The motion files were generated for step changes in aircraft forcing parameters (angle of attack, side-slip angle, and angular rates). These files define the rotations and displacements at discrete time instants and Cobalt then interpolates motion data using cubic-splines and moves the grid for each computational time step. The grid undergoes only translation motion for $\alpha$ and $\beta$ responses, where the relative velocity between grid and flow at each time instant defines the angle of attack and side-slip. For angular rate responses, the motions start from a steady-state solution with zero degrees angle of attack and side-slip angle. The grid then rotates and translates simultaneously. The rotation corresponds to a unit step change in the angular rate, while the translation motion is used in order to keep angles of attack and side-slip zero during rotations.

In angle of attack response simulations the solutions start from a steady-state condition at an angle of attack of $\alpha_{i}$ and a Mach number of $M_{i}$, and then performs a small step in the angle of attack for all $t>0$. In these calculations, $M_{i}$ and $\alpha_{i}$ values correspond to the samples shown in Fig 39, and the side-slip angle is zero degrees at all times and the grid does not rotate at any time. The response functions are then computed by taking the differences between time-varying forces and moments occurring after the step and the steady-state solution at $\alpha=\alpha_{i}$ degrees, and dividing them by the magnitude of the step $(\Delta \alpha)$. For a weakly nonlinear system, the response will be nearly independent of the step magnitude (assuming that $\alpha_{i}+\Delta \alpha \leq \alpha_{i+1}$ ). The step value used was a unit step.

The lift and pitch moment indicial responses to a unit step change in the angle of attack from $\alpha=0$ are shown in Fig. 40 (a) and (b) for Mach numbers of 0.1, 0.3, and 0.5. The lift and pitch moment are plotted against the nondimensional time $s=2 \mathrm{Vt} / \mathrm{c}$. Fig. 40(a) shows that the lift responses have a peak at $s=0$. Likewise, the pitch moment predicts a negative peak at this time as shown in Fig. 40(b). As the steady flow around the vehicle is disturbed by the grid motion, a compression wave and an expansion wave are formed on the lower and upper surfaces of the vehicle that cause a sharp peak in the responses [27]. As the response time progresses, the waves begin to move away from the vehicle, the lift starts to fall, and the pitch moment starts to increase, and then the responses asymptotically reach the steadystate values. Note that final time responses match with the static data slopes at zero angle of attack. Fig. 40(a) and (b) also shows that the initial peak becomes smaller for compressible flow. An explanation is given by Leishman [106]; this is due to the propagation of pressure disturbances at the speed of sound, compared to the incompressible case, where the disturbances propagate at infinite speed.

Fig. 40(c) and (d) shows the lift and pitch moment responses at different angles of attack at Mach number of 0.3 . These figures show that the initial values of responses are invariant with angle of attack, but the transient trend and steady state values change depending on the angle of attack. The changes in the indicial functions are small since the lift and pitch moment are in the nearly linear regime of angle of attack.

The lateral loads response to a unit step change in the side slip angle at different angles of attack are shown in Fig. 41 for Mach numbers of $0.1,0.3$, and 0.5 . In these simulations, the solution starts from a steady-state condition at zero degrees side slip angle and an angle of attack of $\alpha_{i}$ at a Mach number of $M_{i}$, and then iterates such that the side slip angle is held constant to one degree and angle of attack is held constant to $\alpha_{i}$ for all $t>0$, where $M_{i}$ and $\alpha_{i}$ correspond to the samples shown in Fig. 39. Likewise, for the lift and pitch moment, the initial peaks in lateral responses become smaller for compressible flow. Fig. 41 shows that side-force responses $\left(C_{y \beta}\right)$ remain almost unchanged with the changes in the angle of attack for the range of angles studied. This figure shows that the yaw moment responses $\left(C_{n \beta}\right)$ slightly change with the changes in the angle of attack, but significant differences are found for the roll moment. The differences become more apparent as the Mach number increases.

Typically, the angle of attack effects are negligible for the responses due to the angular rates at low to moderate angles of attacks. Fig. 42(a) and (b) shows the lift and pitch moment responses respectively with a unit step change in pitch rate for Mach numbers of 0.1, 0.3, and 0.5. Again there is an initial jump in lift as the grid starts to rotate, and the value decreases as Mach number increases. The lift response starts to fall a short time after initial excitation and then it asymptotically reaches a steady-state value, the so-called pitch dynamic derivative. Fig. 42(a) and (b) shows that increasing Mach number results in the slight decrease of the lift and pitch damping derivatives. These calculations, along with a time-dependent surrogate model, were used to estimate the functions of $C_{L q}(t, M)$ and $C_{m q}(t, M)$ in the ROM equations. Also, the indicial functions with respect to roll and yaw rates are shown in Figs. 43 and 44, respectively. These calculations, along with a time-dependent surrogate model, were used to estimate the functions of $C_{Y p}(t, M), C_{l p}(t, M), C_{n p}(t, M)$, $C_{Y r}(t, M), C_{l r}(t, M)$, and $C_{n r}(t, M)$ in the ROM equations.

The response modeling equations were used for prediction of two SACCON maneuvers: a half Lazy-8 [164] and an Immelmann turn [165]. In both maneuvers, the aircraft enters and terminates the maneuver from a straight and level condition. The maneuvers were generated using the DIDO code [166] to minimize the final maneuver time subject to vehicle aerodynamics, as well as mass properties, state, and control constraints. The angle of attack and side slip angles are limited to $\left[-10^{\circ}, 10^{\circ}\right]$ while the maximum Mach number is 0.5 . Fig. 45 shows that the Lazy- 8 maneuver makes a $180^{\circ}$ turn. The airplane starts a climb steeply to reduce

a

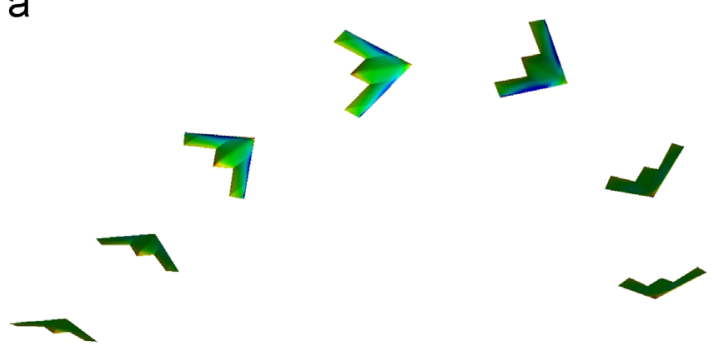

b

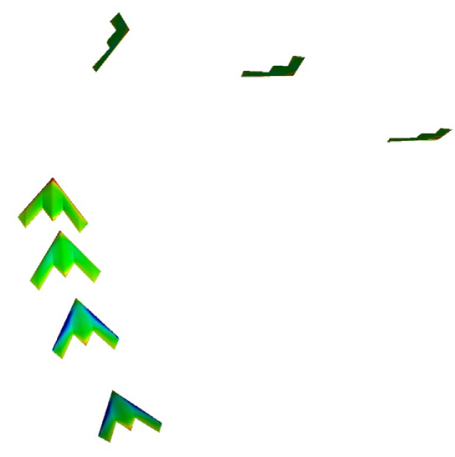

Fig. 49. Surface pressure solutions during Lazy- 8 and Immelmann Turn maneuvers: (a) half Lazy-8 and (b) Immelmann turn. 
flight speed as shown in Fig. 45(b). This reduced speed helps to have a smaller radius turn and total traveled time [165]. Next, the airplane starts to roll as the pitch angle decreases, where at $90^{\circ}$ yaw angle, the vehicle is at zero pitch and maximum roll angle as shown in Fig. 45(d). This is followed by a descent trajectory and decreasing roll angle, increasing pitch angle, and increase in the speed until the vehicle reaches the initial velocity and altitude. The Immelmann turn as shown in Fig. 46 comprises a half loop with a half roll at the end. The maneuver starts with a steep climb and thus decreases the speed as shown in Fig. 46(b). At the maximum

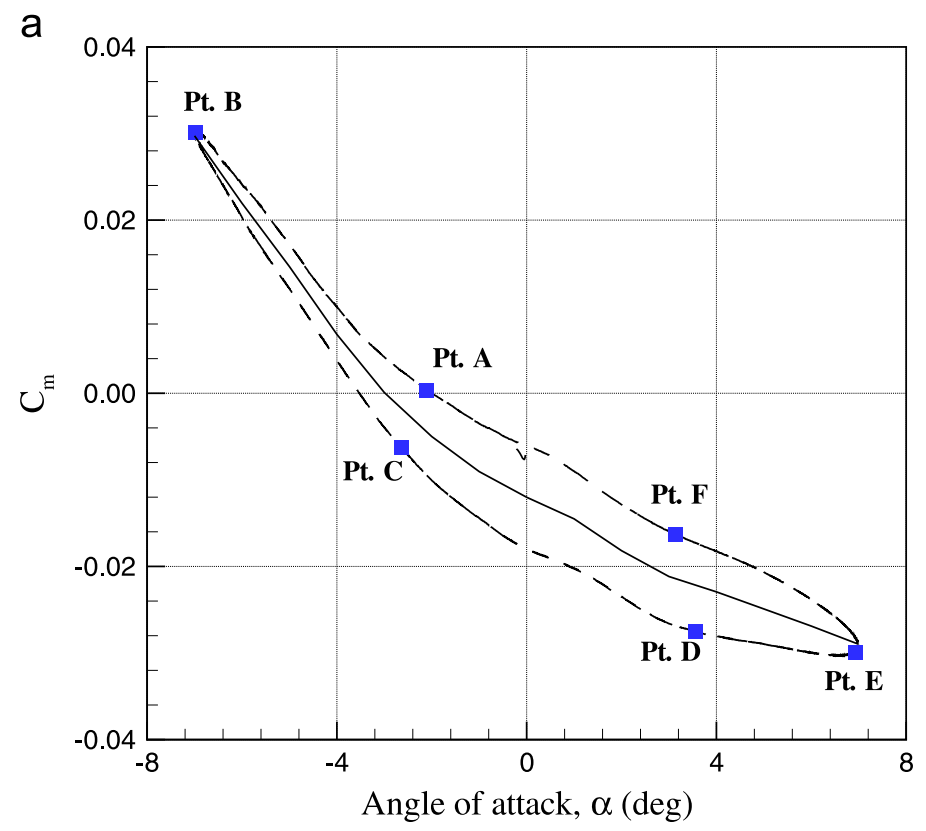

b
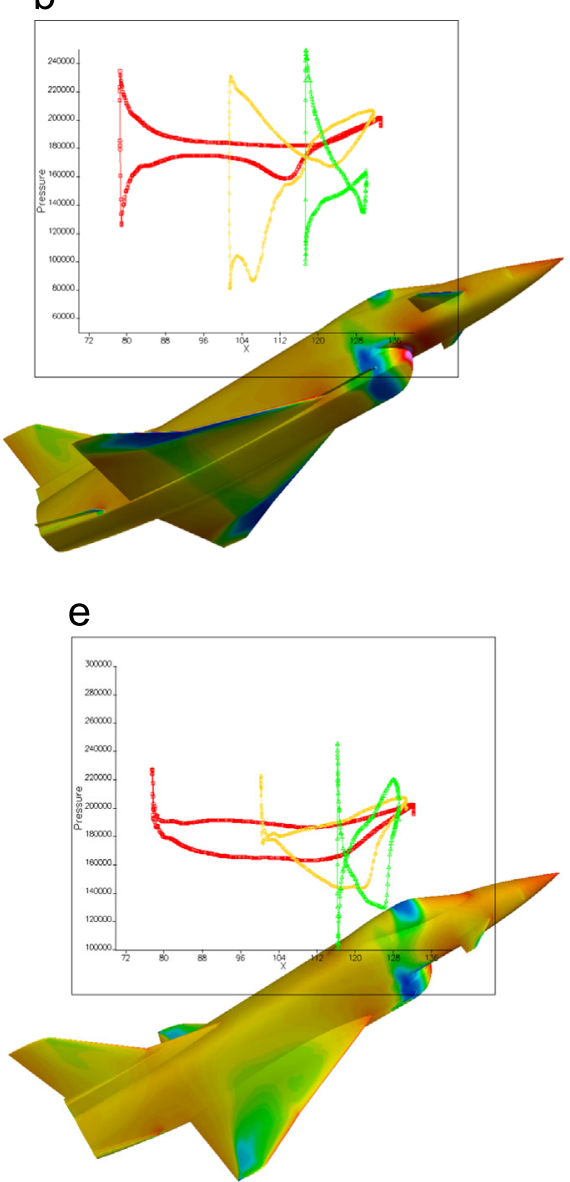

C
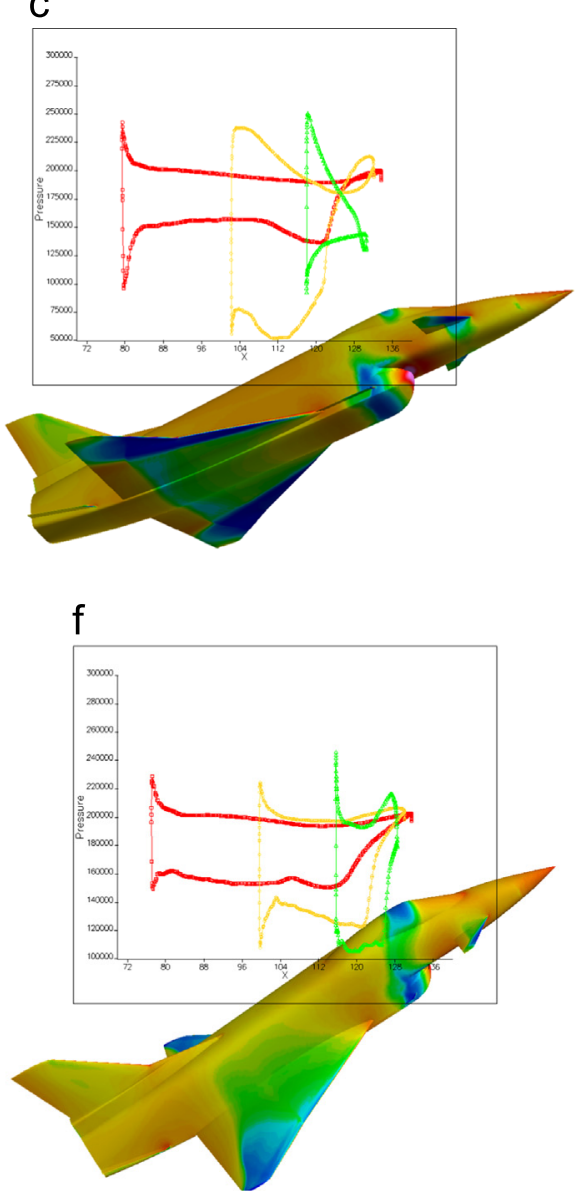

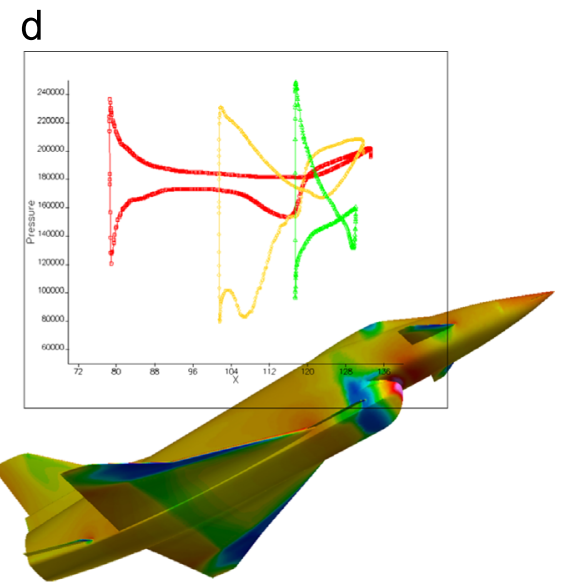

g

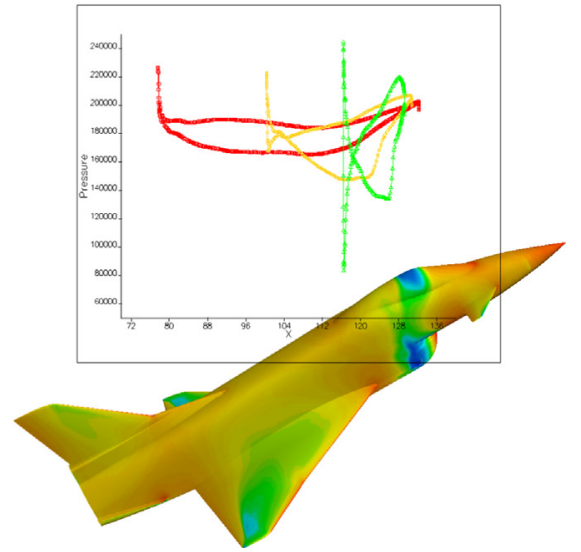

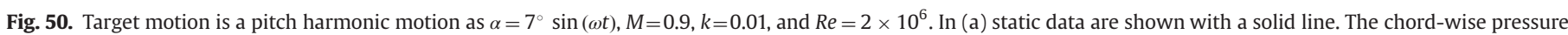

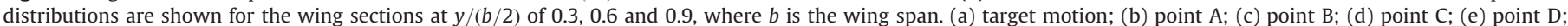
(f) point $\mathrm{E}$; and (g) point $\mathrm{F}$. 
pitch angle, the aircraft heading suddenly changes from $0^{\circ}$ to $180^{\circ}$, which makes the aircraft final flight path exactly opposite of the initial path. As the heading starts to increase, the aircraft performs a half roll to level the wing as shown in Fig. 46(d). The final altitude is slightly higher than the starting altitude as shown in Fig. 46(a).

The time-marching simulations of these maneuvers were calculated using RANS simulations with grid motion. The computations start from a steady-state solution corresponding to the initial state of the maneuver and then advance in time using second-order temporal accuracy. The grid moved and rotated at each time step according to the maneuver descriptions shown in Figs. 45 and 46. Cobalt reports time-dependent aerodynamic forces and moments in an inertia axis; these forces and moments are then transformed to the wind axis and normalized by the reference area and length and the dynamic pressure at each time step. Fig. 47 depicts the predicted aerodynamic loads of half Lazy- 8 maneuver. The comparisons between the created ROM with the time-marching model show good agreements for all

Table 4

Comparison of model costs and errors to predict the X-31 target motion at $M=0.9$.

\begin{tabular}{|c|c|c|c|}
\hline & $\operatorname{Cost}^{\mathrm{a}}$ & Error (\%) & Comments \\
\hline Volterra using spiral & 21,500 & 1.42 & Limited to the frequency of maneuver \\
\hline Volterra using chirp & 21,500 & 3.47 & Might be applied to other frequencies \\
\hline RBF using spiral & 21,500 & 3.24 & Limited to the frequency of maneuver \\
\hline RBF using chirp & 21,500 & 1.10 & Might be applied to other frequencies \\
\hline RBF using Schroeder & 21,500 & 1.53 & Might be applied to other frequencies \\
\hline Linear indicial function & 768 & 12.67 & Small amplitude motions with any frequency \\
\hline Nonlinear response function & 5400 & 0.48 & Any amplitude ${ }^{\mathrm{b}}$ and frequency \\
\hline SBRF in $\alpha-M$ space & 344,000 & 0.98 & Any amplitude, Mach $^{\mathrm{b}}$ at a fixed frequency \\
\hline Response function in $\alpha-M$ space & 27,000 & 0.48 & Any amplitude, Mach ${ }^{\mathrm{b}}$ and frequency \\
\hline
\end{tabular}

a CPU hours.

${ }^{\mathrm{b}}$ Amplitude and Mach number should be within the range of input space.

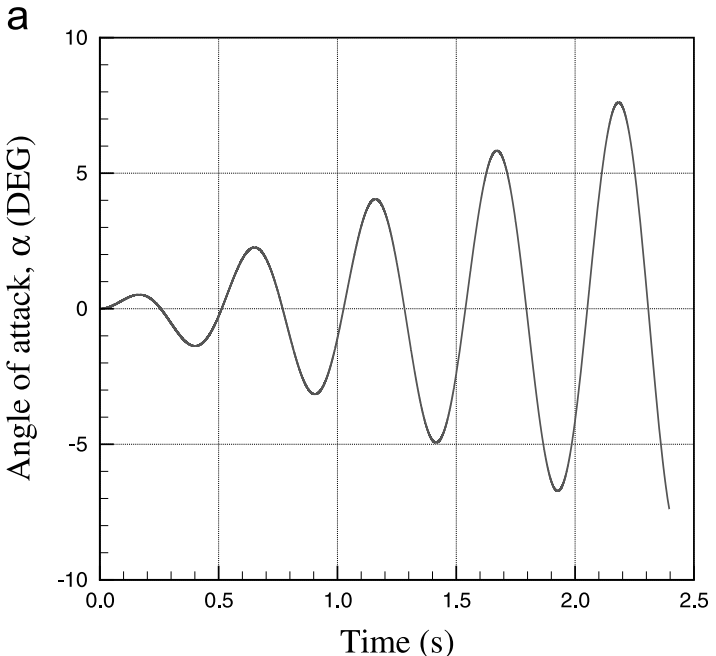

$b$

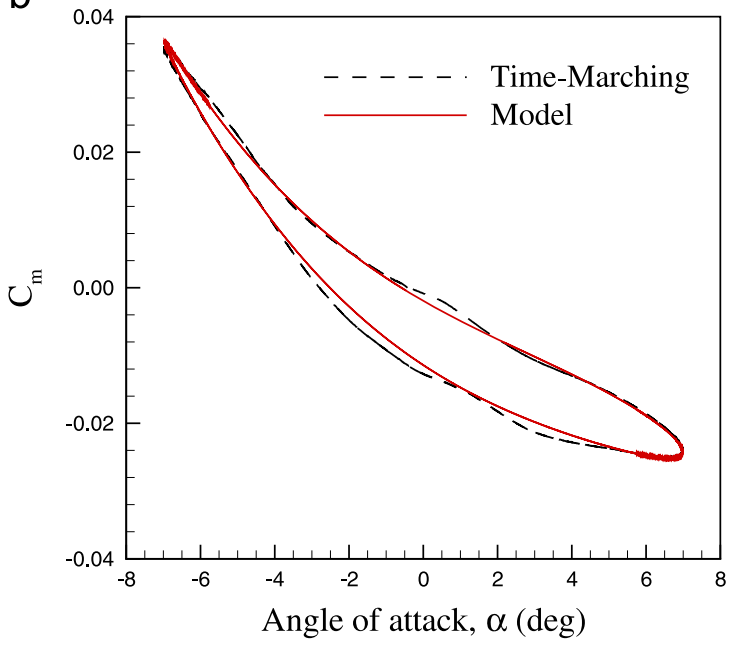

C

d
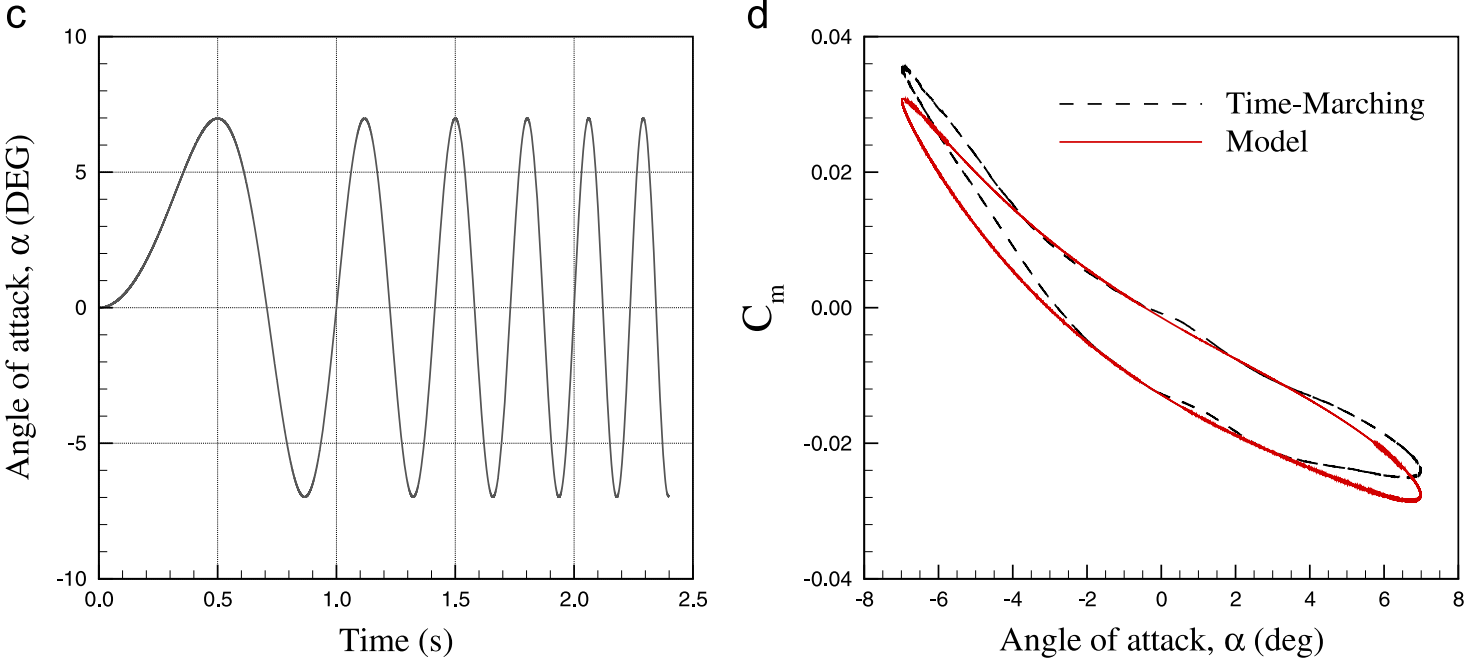

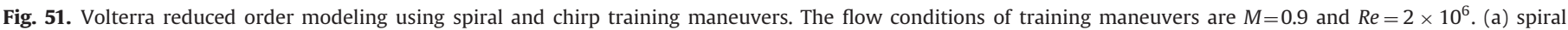
maneuver; (b) ROM prediction; (c) chirp maneuver; and (d) ROM prediction. 
coefficients. The predictions of aerodynamic loads for the Immelmann turn are also shown in Fig. 48. Again, the ROM is in close agreement with the time-marching model for all coefficients; small discrepancies are found in the roll and yaw moments for maneuver times between 12 and $16 \mathrm{~s}$, where the angle of attack is negative. This shows that the
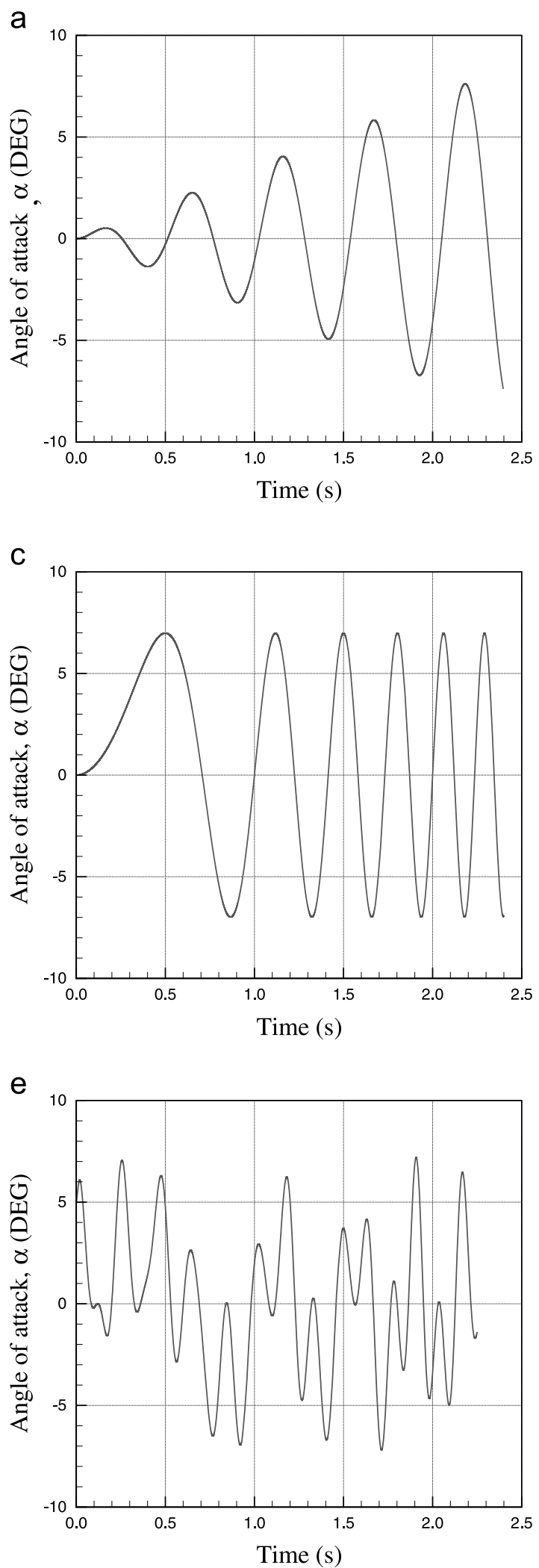

roll and yaw moments of SACCON are not symmetric about zero degrees angle of attack and hence the indicial functions need to include negative angles of attack as well. Note that the cost of generating each time-marching model is approximately 50,000 CPU hours using 256 processors $(2.7 \mathrm{GHz})$, but the model predictions are

b

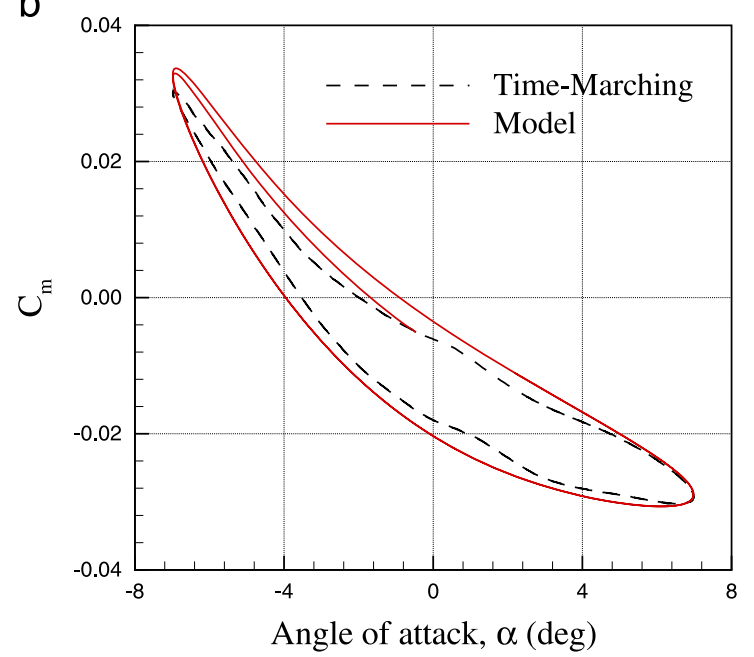

d

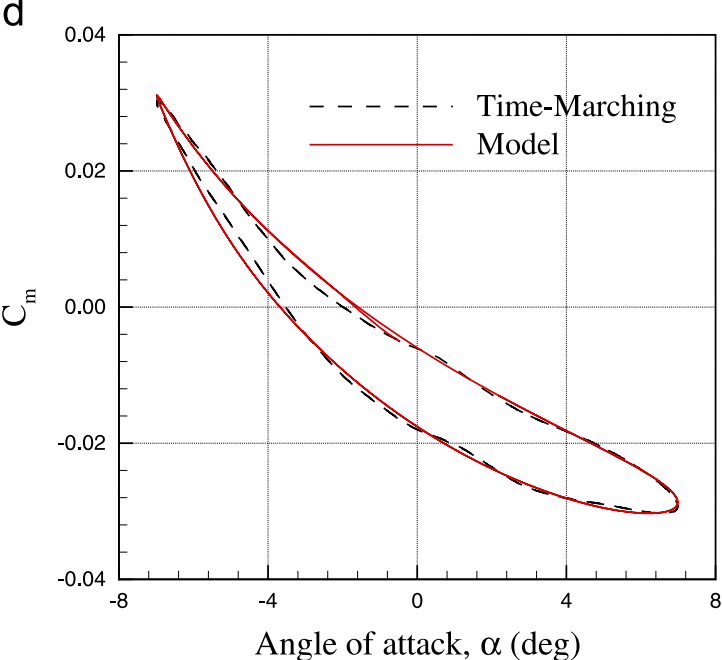

f

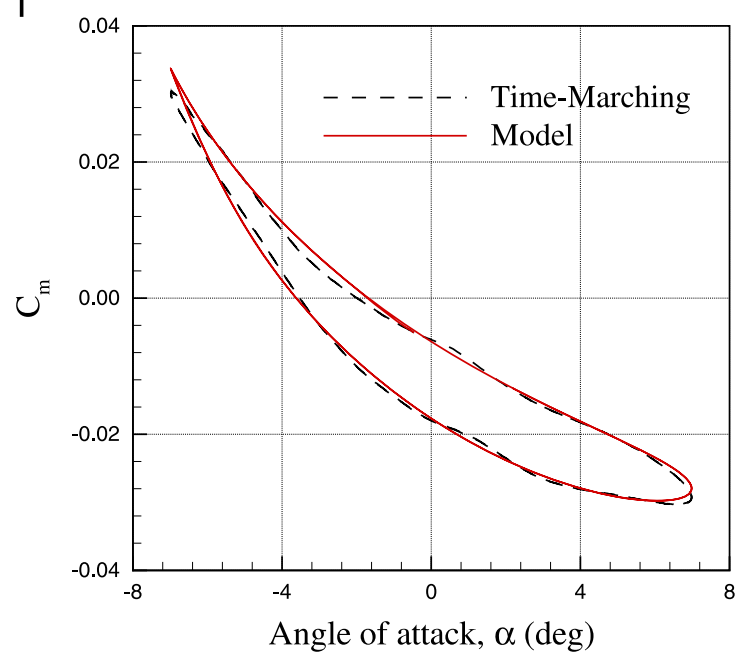

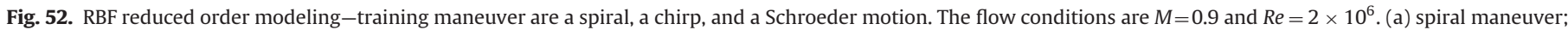
(b) ROM prediction; (c) chirp maneuver; (d) ROM prediction; (e) Schroeder maneuver; and (f) ROM prediction. 
generated within a few seconds. Finally, the surface pressure distributions during maneuvers are shown in Fig. 49. The leading edge vortex can be seen behind the leading edge on the upper wing around 10 angle of attack during the maneuvers.

\subsection{X-31 aerodynamics modeling}

All ROMs considered were evaluated for a similar target motion to permit direct comparison of costs and errors. The objective of all models is to predict the unsteady pitch moment resulting from a sinusoidal pitch oscillation at a freestream Mach number of 0.9 and reduced frequency of $k=0.01$. The amplitude of oscillation is held constant at $7^{\circ}$ and the mean angle of attack is zero degrees. Fig. 50(a) shows the computed pitch moment coefficient, $C_{m}$, by solving the RANS and SARC-DES turbulence model equations in a time-accurate fashion. The cost of simulating three pitch cycles is approximately $13,000 \mathrm{CPU}$ hours using 256 processors $(2.7 \mathrm{GHz}$ ).

Fig. 50(a) shows that the pitch moment curve of target motion makes a nonlinear loop on the moment versus angle of attack figure due to the occurrence of shock waves and vortices. This figure also shows that the pitch moment curve is not symmetric about zero degrees angle of attack. The moment curve shows a negative slope during the pitch cycle such that it has more negative slope values at negative angles of attack compared with the slope values at positive angles of attack. Some flow features

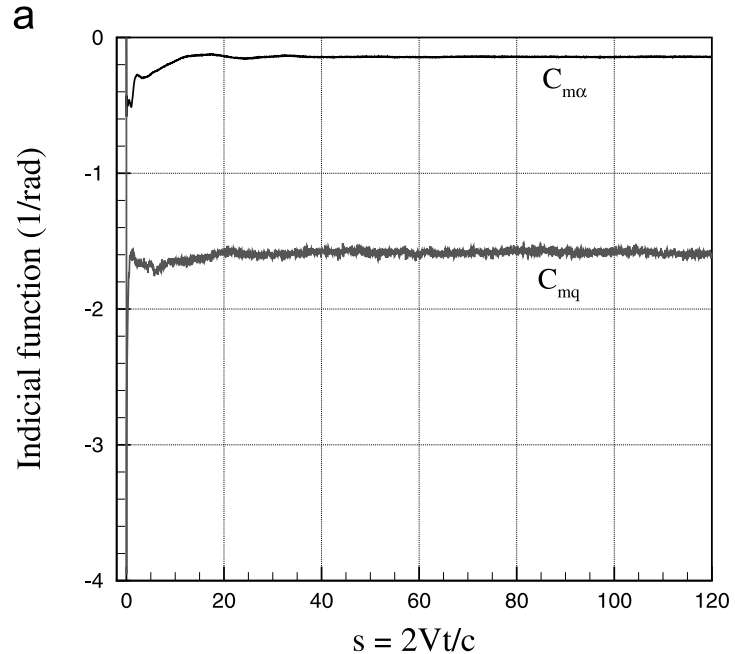

C

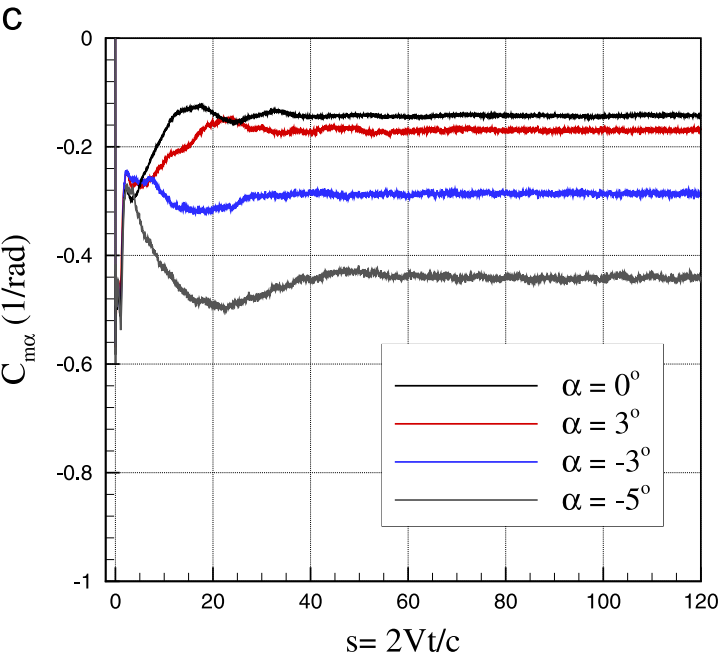

during the pitch oscillation are shown in Fig. 50(b)-(g) and will be briefly discussed here. In Fig. 50(b) the angle of attack is $-2.1^{\circ}$ and a vortex can be seen emanating from the wing root on the lower surface which spirals towards the wing tip. This vortex causes a sharp negative pressure peak to occur close to the wing leading edge as shown in the surface pressure plots of Fig. 50(b)-(d). Fig. 50(b) also shows that a shock wave is formed on the lower surface of the wing which is nearly perpendicular to the fuselage before it interacts with the leading edge vortex. At the minimum angle of attack in the pitch cycle, i.e. $\alpha=-7^{\circ}$, the leading edge vortex becomes much stronger and the wing surface pressure close to the leading edge drops further as shown in Fig. 50(c). This figure also shows that as the angle of attack becomes smaller, the shock moves downstream and therefore changes the pitch moment curve slope. No vortices were observed on the wing during pitching at positive angles of attack, but a vortex was formed on the canard tip at the maximum angle of attack in the pitch cycle, i.e. $\alpha=7^{\circ}$, as shown in Fig. 50(f). Fig. 50(e)-(g) shows that a shock wave is formed over the upper surface which is no longer perpendicular to the fuselage and moves slowly with increasing in the angle of attack during upstroke. The model costs and errors to predict this target motion are summarized in Table 4 with more details given below:

For the identification of the Volterra kernels, the chirp and spiral training maneuvers were generated using CFD (RANS and

\section{b}

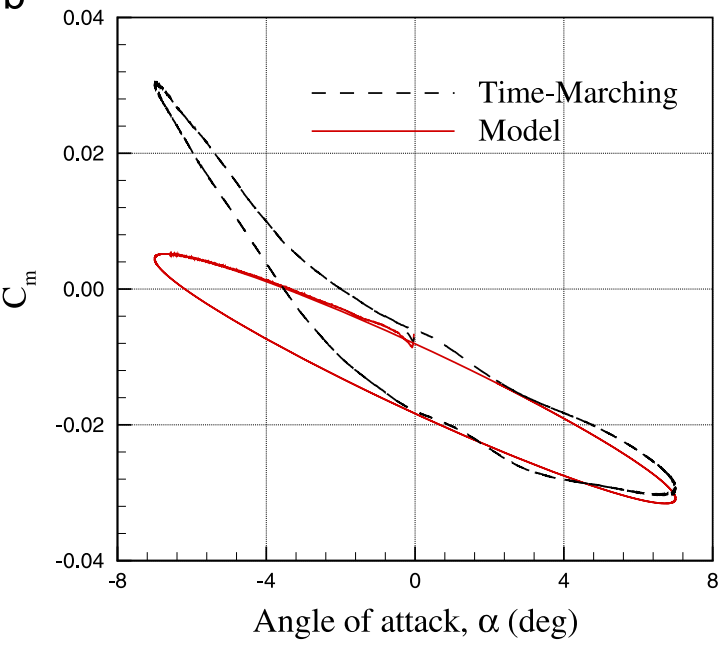

d

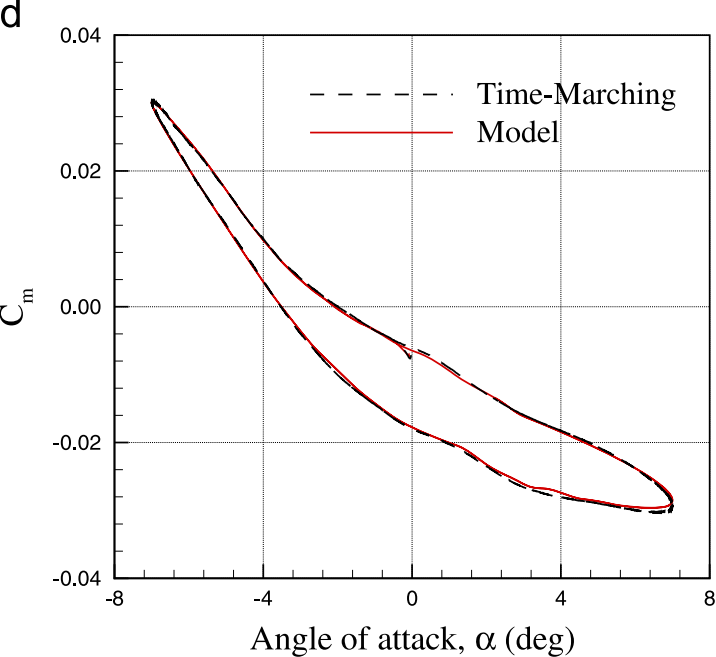

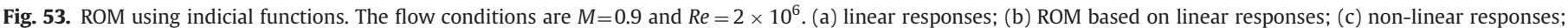
and (d) ROM based on non-linear responses. 
SARC-DES turbulence model) as the source of the data. The variations of angle of attack with time for these maneuvers are shown in Fig. 51(a) and (c). Both maneuvers ran for $2.4 \mathrm{~s}$ of physical time and started from a steady-state solution. The chirp maneuver has an amplitude of $7^{\circ}$ starting from zero degrees angle of attack and pitching with a frequency of $1 \mathrm{~Hz}$ at $t=0$. The chirp motion frequency increases linearly with time. The spiral maneu-

a

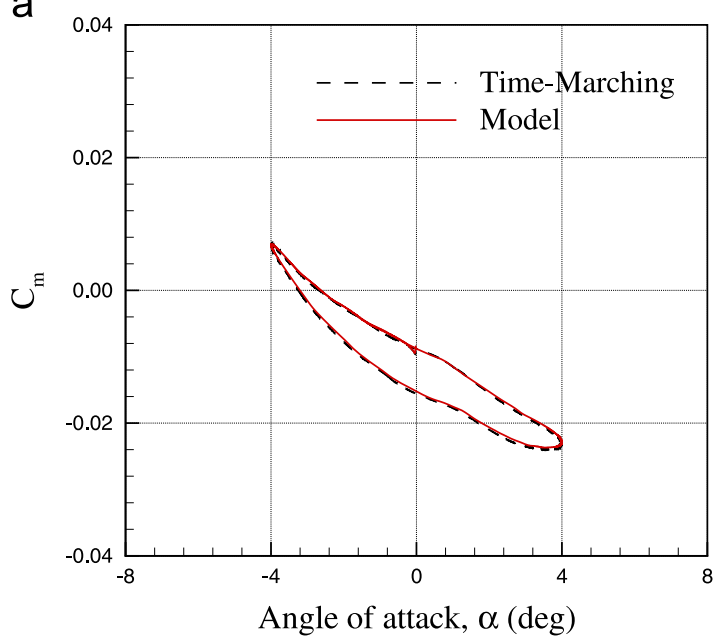

C

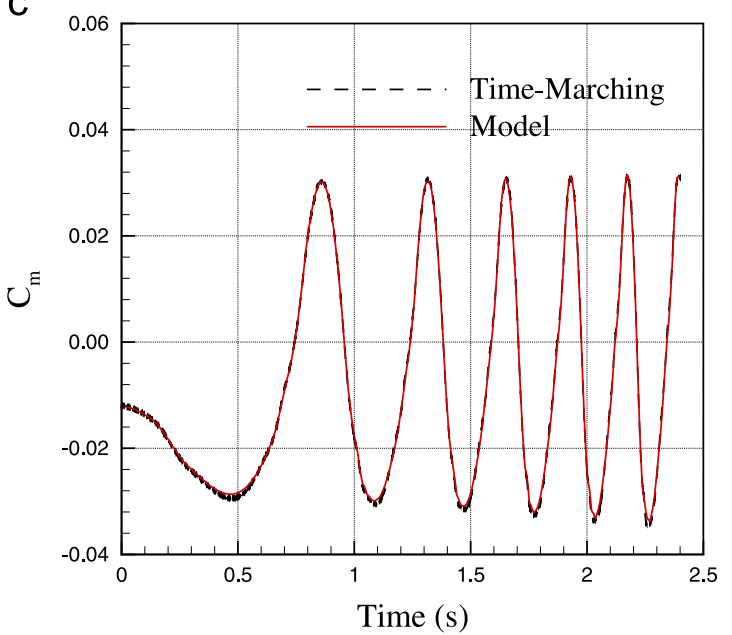

e

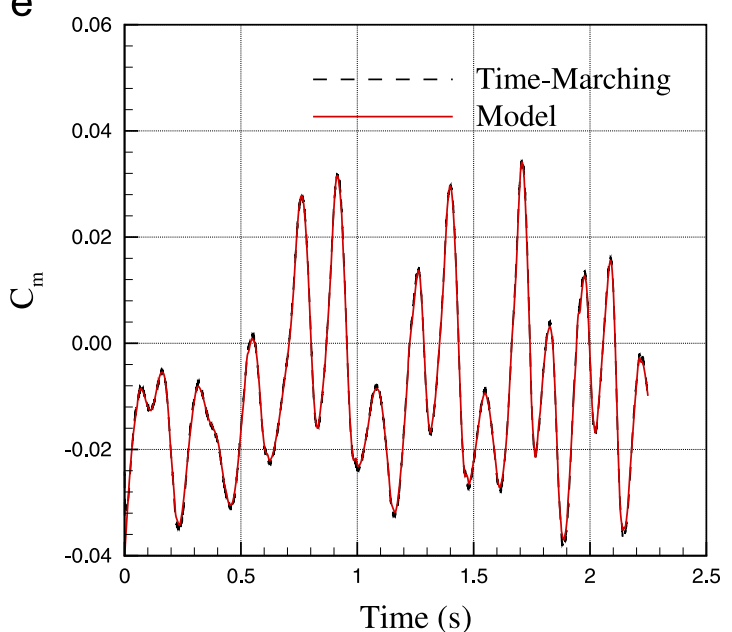

ver has an initial amplitude of $3.5^{\circ}$, starting from zero degrees angle of attack and pitching at constant frequency of $k=0.01$. The oscillation amplitude in the spiral motion increases as time progresses. Note that the spiral maneuver is at the reduced frequency of the maneuver to be predicted. The cost of generating each training maneuver is approximately 21,500 CPU hours using 256 processors.

b

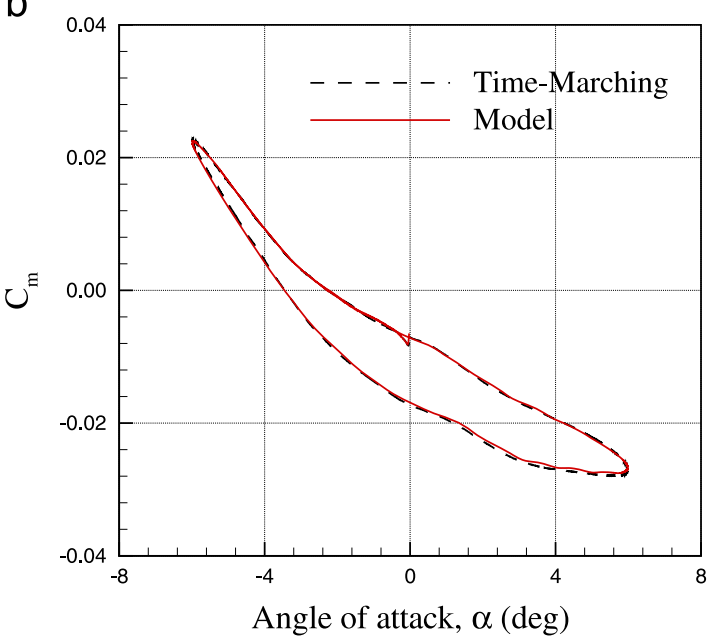

d

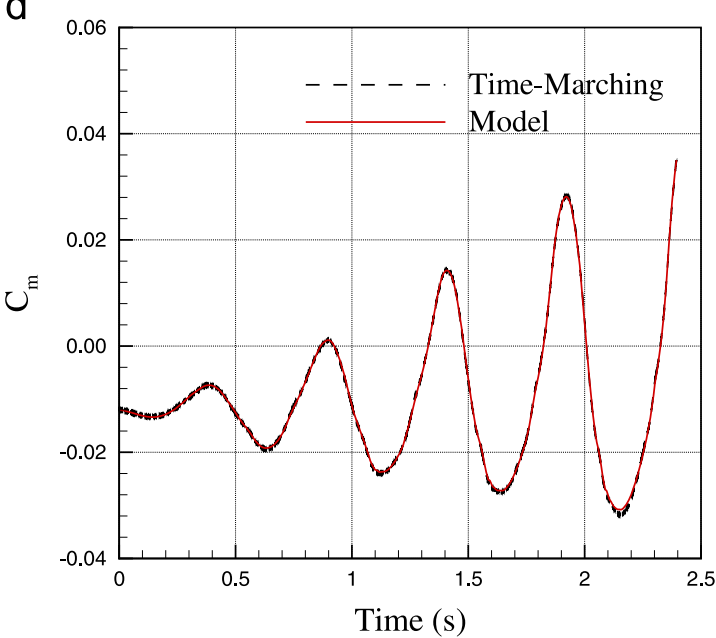


The first and second order kernels of the Volterra model were estimated from time-history simulations of chirp and spiral training maneuvers. These estimations were used next to predict the target motion. The ROM predictions based on spiral and chirp training maneuvers are compared with target data in Fig. 51 (b) and (d). The comparisons show a good agreement with CFD data for a ROM identified from spiral data (error norm is $1.42 \%$ ), but the ROM identified from chirp data does not match everywhere, in particular, around the maximum and minimum angles of attack. The instantaneous frequency in the chirp maneuver varies with time and hence it might not have sufficient information to identify the Volterra kernels corresponding to a swept amplitude motion at constant frequency. However, the ROM based on chirp data possibly could be used for predicting aerodynamic responses from pitch oscillations at other frequencies within the range of input, but the ROM based on the spiral is possibly only valid for the motions at a fixed reduced frequency.

The generated chirp and spiral training maneuvers were also used to find a mapping between the pitch moment coefficient and the instantaneous pitch motion variables. This mapping was next learned using a RBF neural network. Also, a Schroeder maneuver was defined by a multi-stage frequency sweep. This maneuver started from an initial angle of attack of $4.95^{\circ}$. The number of frequencies in the maneuver, $N$, was set to 20 with an initial amplitude of $7^{\circ}$. This maneuver ran for $2.4 \mathrm{~s}$ of physical time as well and is shown in Fig. 52(e). The aircraft responses to these three maneuvers were generated using the URANS equations and the SARC-DES turbulence model. The training data were next normalized using the mean and standard deviation of each input. The data were then rearranged according to Eq. (30) and the RBF network performance was tested for different values of $m$ and $n$, with a performance error threshold of $1 \times 10^{-6}$. All networks computed converged to the threshold error. The results showed that using $m=n=4$ is sufficient for modeling the motions studied. The trained networks were then tested against the target motion; the ROM predictions are shown in Fig. 52(b), (d) and (f). These figures show that the predicted ROM values agree well with the time-marching solution, although the ROM based on chirp and Schroeder maneuvers showed better accuracy than models based on the spiral maneuver.

The indicial pitch moment responses of the X-31 aircraft with a unit step change in angle of attack and pitch rate are shown in Fig. 53(a). These functions correspond to the fixed Mach number of 0.9. In $C_{L \alpha}$ simulations, the angle of attack is zero degrees at $t=0$ and is held constant to one degree for all other times. In $C_{L q}$ simulations, the grid starts to pitch up with a normalized pitch rate of $q=1 \mathrm{rad}$ at $t=0$ and the angle of attack is held to zero degrees during simulations with the aid of grid translation. All computations started from a steady-state solution and then are advanced in time using second-order temporal accuracy with five Newton subiterations. As shown in Fig. 53(a), the pitch moment responses have a negative peak at $t=0$ followed by an increasing trend. As the response time progresses the pitch moment responses start to increase and then asymptotically reach the steady-state values. The cost of generating each indicial function is around 384 CPU hours using 256 processors. This model has much lower cost than RBF and Volterra theory, because response functions reach the steady state solutions in one order of magnitude less time than the time used to complete training maneuvers.

A linear ROM was created using Eq. (23) and used for prediction of target maneuver. The results are compared with timemarching model in Fig. 53(b). The figure shows that linear ROM fails to accurately predict the pitch moment values at all angle of attack. The error norm value is $12.67 \%$. The functions of $C_{L \alpha}$ vary largely with angles of attack in the transonic speed range and thus a linear ROM cannot predict these effects.
Next, the X-31 $C_{L \alpha}$ functions were simulated at different angles of attack and at a freestream Mach number of 0.9 and are shown in Fig. 53(c). Note that the pitch moment slope is not symmetric with zero degrees angle of attack and hence the simulations included both positive and negative angle of attack responses. The total cost of generating a nonlinear ROM is now increased to approximately 5400 CPU hours (this is still cheaper than the cost of simulating chirp, spiral and Schroeder maneuvers).

Fig. 53(c) shows that the responses at the initial time are invariant with angle of attack, but the intermediate and final values change depending on the angle of attack. Fig. 53(c) shows that the pitch moment responses have more negative values than positive angles of attack due to vortex formation on the lower surface of the wing. A nonlinear ROM was created, and then using a linear interpolation scheme, the prediction of the target maneuver was evaluated. Fig. 53(d) shows that the nonlinear ROM predictions agree very well with the time-marching simulation values with an error of $0.48 \%$.

Such a nonlinear ROM could be used for computing the pitch moment responses from many other motions with different amplitudes and frequencies. For example, the ROM was used to predict two pitch oscillations with $4^{\circ}$ and $6^{\circ}$ amplitude at $M=0.9$ and $k=0.01$. The predictions are compared with time-marching solutions in Fig. 54(a) and (b). Again a very good match was found. Also, the ROM predictions were evaluated for the chirp, spiral and

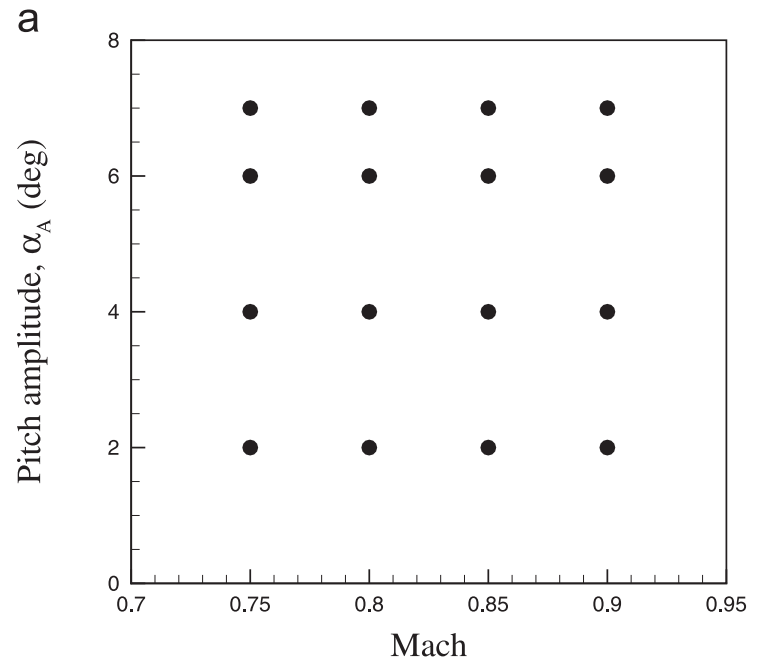

b

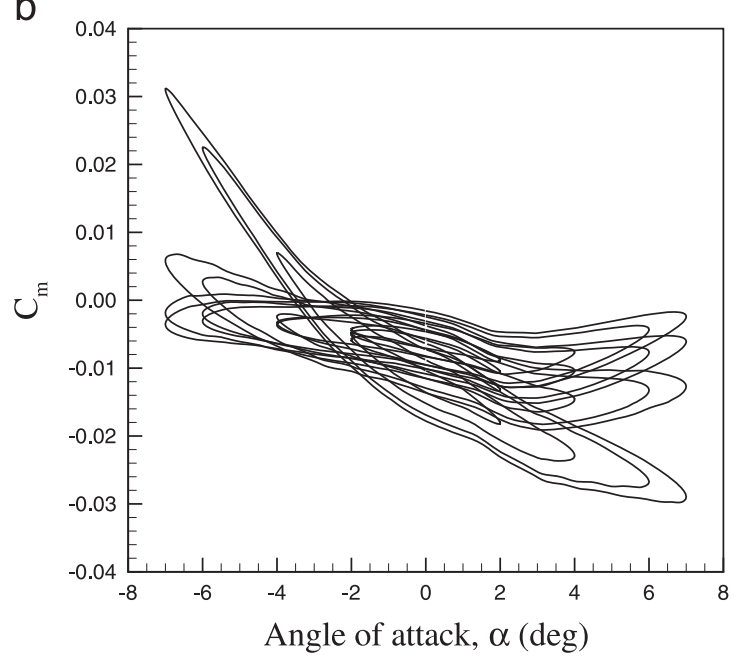

Fig. 55. Samples and training pitch motions for a SBRF model. (a) samples design for SBRF model and (b) training pitch motions for SBRF model. 
Schroeder maneuvers used in RBF work. Fig. 54(c)-(e) shows that even for this varying amplitude and frequency motions, the created ROM matches very well with the CFD data.

For the generation of the SBRF model, time-accurate simulations were pre-computed for various combinations of pitch amplitude and Mach number at a fixed reduced frequency. The two-dimensional parameter space was filled using Design of Experiment methods and is shown in Fig. 55(a). Also, the pitch motion simulations of all samples are shown in Fig. 55(b). The SBRF model was used for the prediction of the pitch moment coefficient time history for sinusoidal forced motions about zero degrees angle of attack, amplitude of $7^{\circ}$ and values of Mach number of $0.78,0.825$, and 0.88 . Model predictions are compared to time-accurate results in Fig. 56. Tests were performed to evaluate the dependency of the model predictions on the number of previous steps in the inputs (angle of attack time history, first and second time derivatives, and Mach number) and output (the prediction itself). No significant dependence was found for values of $m$ and $n$ up to 2. This may be attributed to the small time step increments used in the time-accurate simulations. Although the method robustness could degrade for higher values of $m$ and $n$, it is considered relevant that the predictions are unaffected for a range of values. In this work, the Kriging interpolation was used to approximating the mapping function between inputs and outputs. A good agreement is noted in Fig. 56 for all flight conditions, with the model predictions being generated in few seconds.
An issue regarding the SBRF model is that the cost of simulating three pitch cycles for each sample shown in Fig. 55(a) is around 13,000 CPU hours using 256 processor and the model still cannot predict the aerodynamic responses to motions at other frequencies. A ROM based on indicial functions, along with a time-dependent surrogate approach, is proposed for aerodynamics modeling in the angle of attack/Mach number/frequency space. In this model, the indicial functions in the angle of attack and Mach number space are interpolated from some available samples. A ROM based on these functions is still cheaper than the time-marching model and SBRF model because the indicial functions eliminate the need of repeating calculation for each frequency.

The X-31 motions considered encompass $\alpha$ and $M$ values in the range of $\left[-7^{\circ}, 7^{\circ}\right]$ and $[0.75,0.9]$, respectively. A set of samples including 56 points is defined on the $\alpha$ and $M$ space using factorial design. These points are shown in Fig. 57(a).

The indicial functions with respect to angle of attack are calculated using the CFD and grid motion approach for each sample condition. The pitch rate indicial functions are calculated for a unit step change in the pitch rate for each Mach number shown in Fig. 57(a). The total cost of generating all functions is now approximately 27,000 CPU hours. The calculated indicial functions due to a unit step change in angle of attack are shown in Fig. 57(b) for each Mach number in the sample design. This figure shows that the pitch moment initial, intermediate, and final

b

a

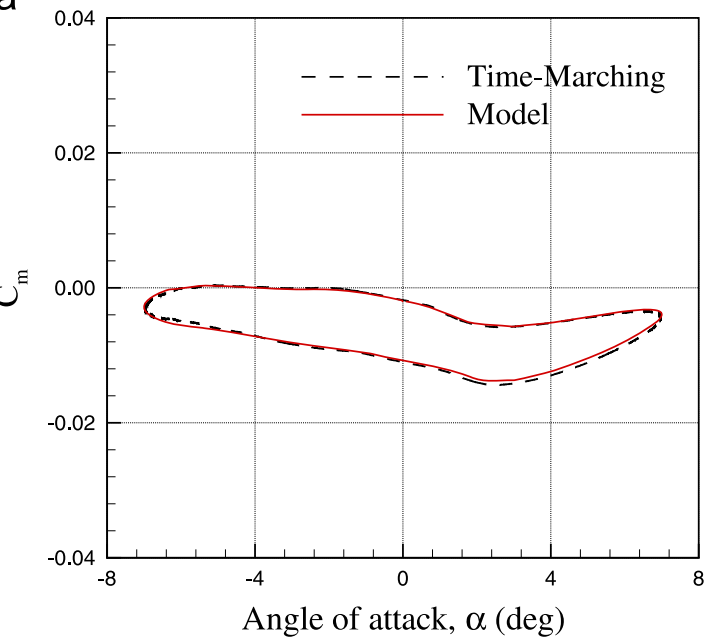

C

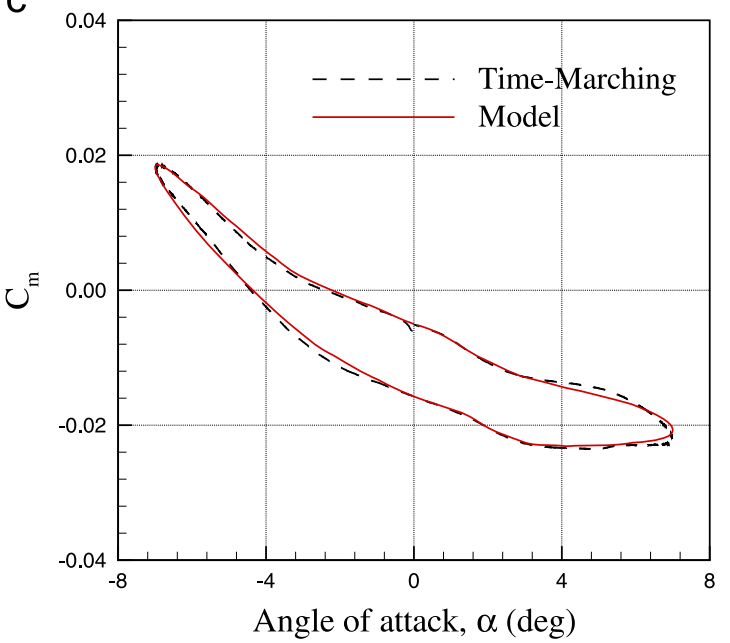

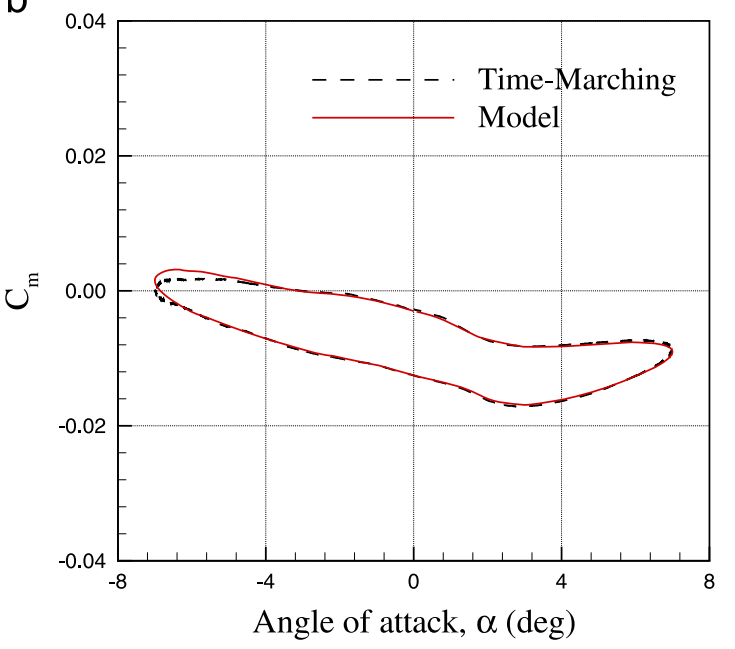


loadings are different at each Mach number. The initial peak in the pitch moment becomes smaller for higher Mach number. Fig. 57 (c) also shows that pitch rate indicial functions decrease as the Mach number increases.

A new ROM is now created along with a time-dependent surrogate model to determine the terms in Eq. (24) at each time step. The validity of the ROM is tested for several motions in the angle of attack/frequency/Mach number space and compared with time-accurate CFD simulations in Fig. 58. This figure shows that the ROM predictions agree well with the CFD data, although small discrepancies are found in the high-speed motions. This is likely due to the sample design used with a uniform spacing and the fact that the pitch moment changes suddenly at high speeds. More samples at high speeds could improve the model predictions.

\section{Conclusions}

Aircraft stability and control analysis using CFD requires a very large number of CFD simulations to determine appropriate forcing parameters within the frequency/amplitude/Mach number space.
Typically, the time-accurate CFD simulations start from a steady state solution and are marched (iterated) in pseudo time within each physical time step using a dual-time stepping scheme. Also, to have a free decay response to the initial grid perturbation, it is often necessary to march time-accurate solutions for several oscillations. The aircraft configurations used in this work have highly swept slender wings resulting in complex vortical flow under various conditions. A highly refined mesh and small time step are required to accurately resolve the unsteady flow around the aircraft in space and time. Because of the combination of large grids and small time steps, and a large number of simulations, the full-order modeling approach is expensive for stability and control analysis of aircraft. This paper reviews the use of reduced order models that significantly reduces the CFD simulation time required to create a full aerodynamics database, making it possible to accurately model aircraft static and dynamic characteristics from a limited number of time-accurate CFD simulations.

The models considered were based on linear and nonlinear indicial response theory, Volterra theory, radial basis functions, and a surrogate-based recurrence framework. The response functions were directly calculated from unsteady RANS simulations

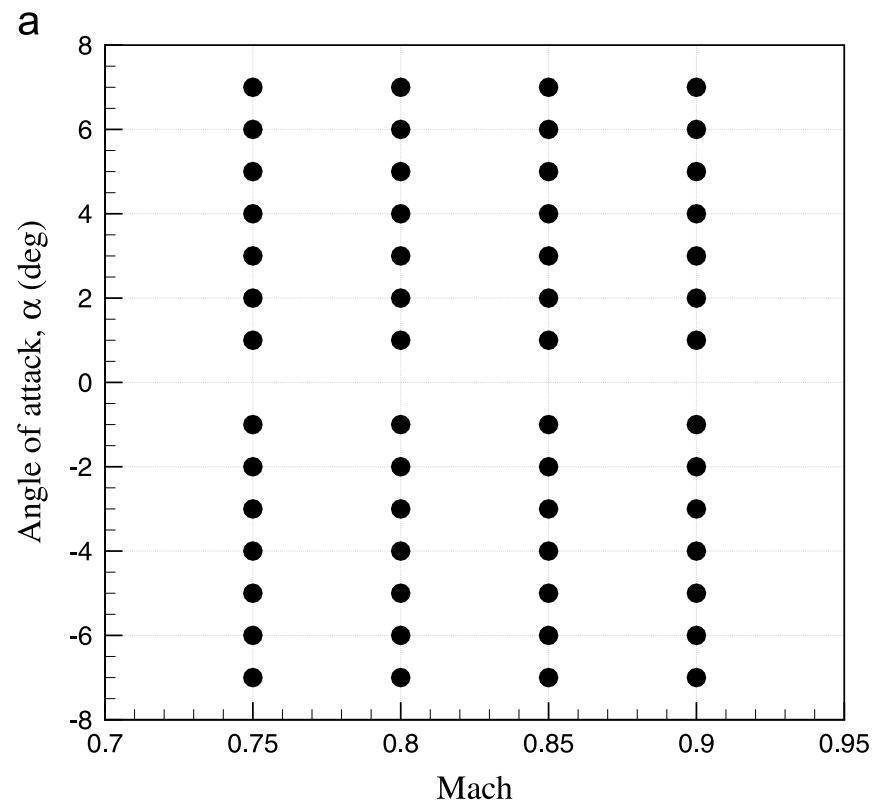

b

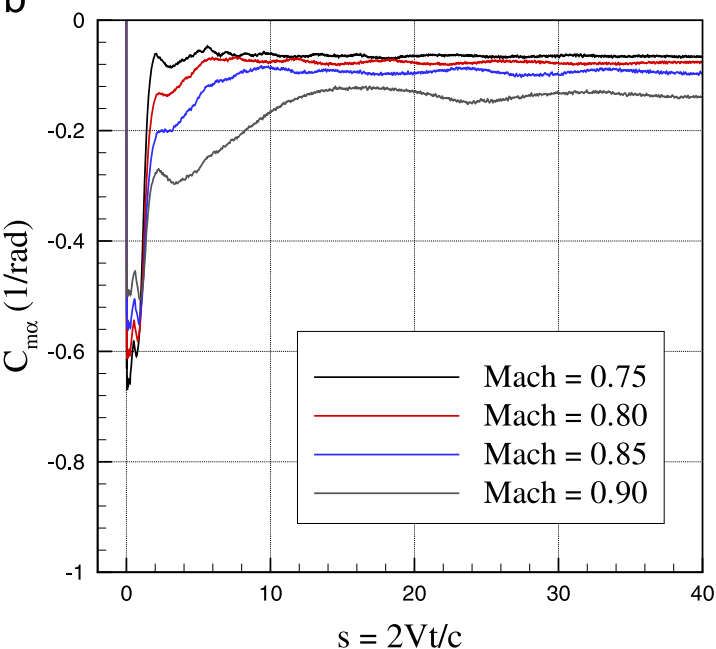

C

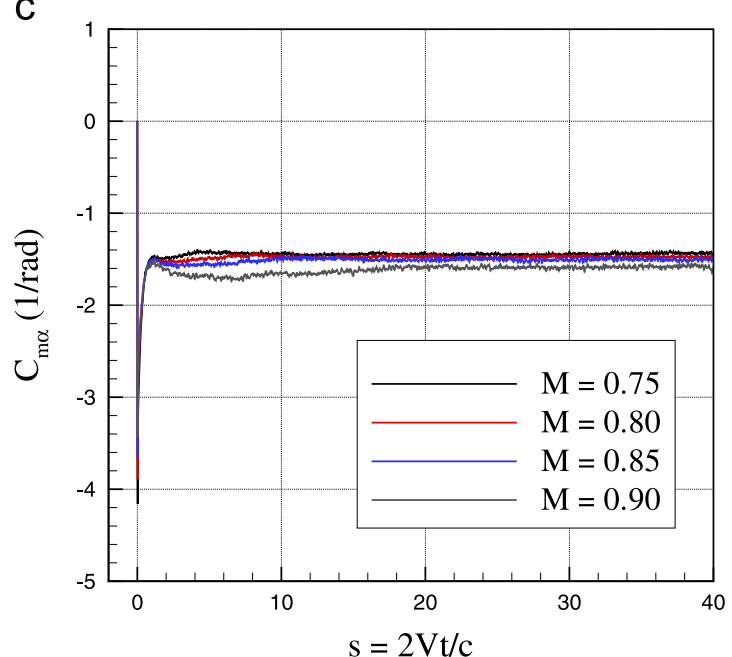

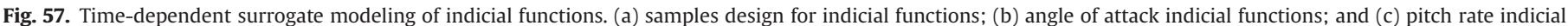
functions. 
starting from an initial steady-state condition with a prescribed grid motion. An important feature of this approach is uncoupling the effects of angle of attack and side-slip from angular rate responses. A method to efficiently reduce the number of step

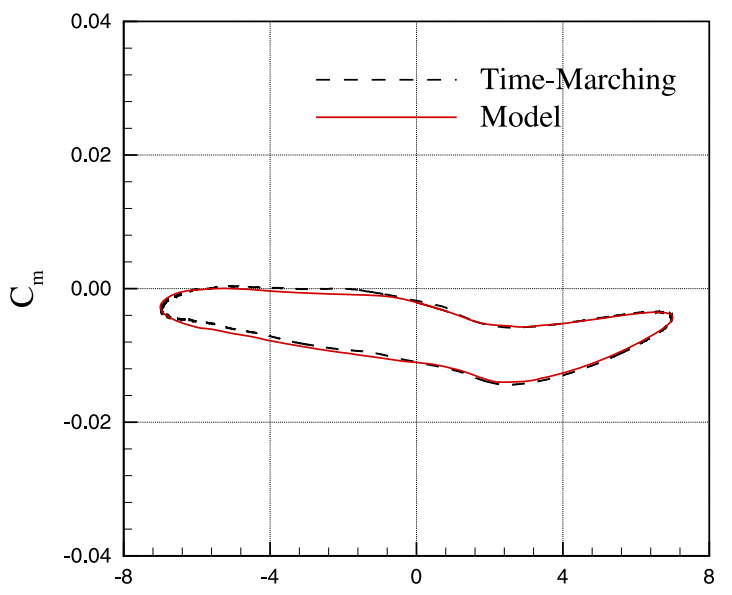

Angle of attack, $\alpha$ (deg)

$\alpha=7^{\circ} \sin (\omega t), k=0.01, \mathrm{M}=0.78$

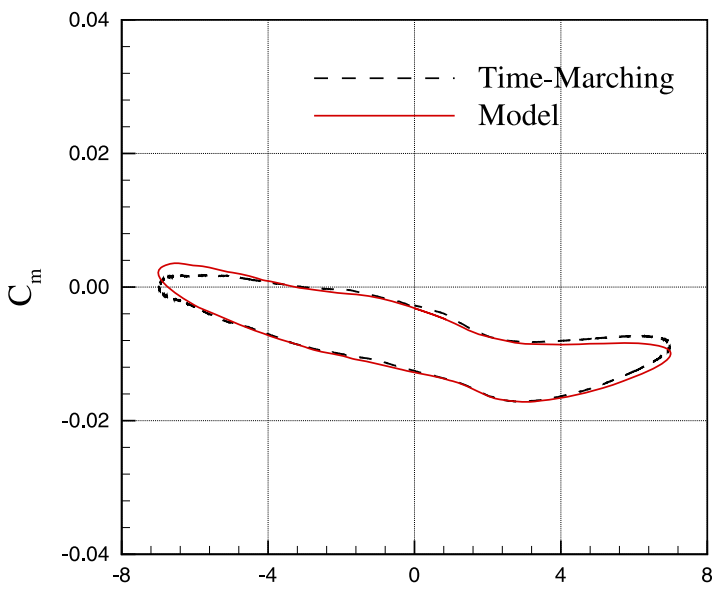

Angle of attack, $\alpha$ (deg)

$\alpha=7^{\circ} \sin (\omega t), k=0.01, \mathrm{M}=0.825$

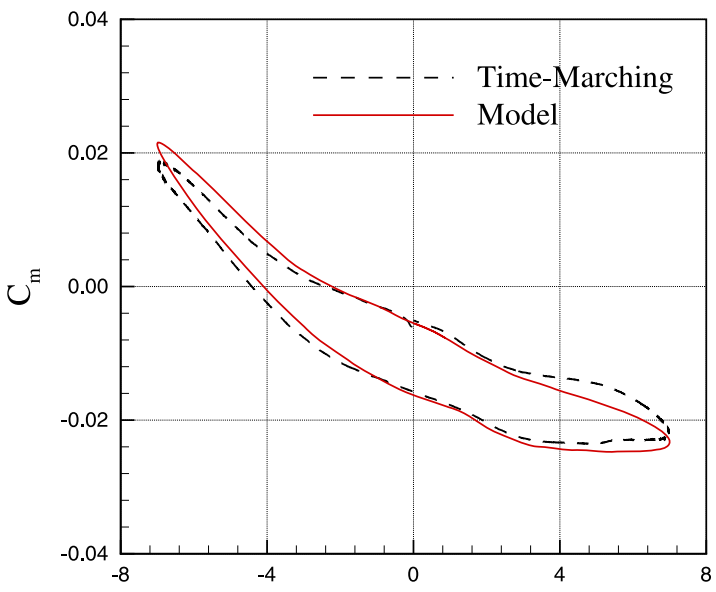

Angle of attack, $\alpha$ (deg)

$\alpha=7^{\circ} \sin (\omega t), k=0.01, \mathrm{M}=0.88$ function calculations within the angle of attack/Mach number space was described. This method uses a time-dependent surrogate model to fit the relationship between flight conditions (Mach number and angle of attack) and step functions calculated for a

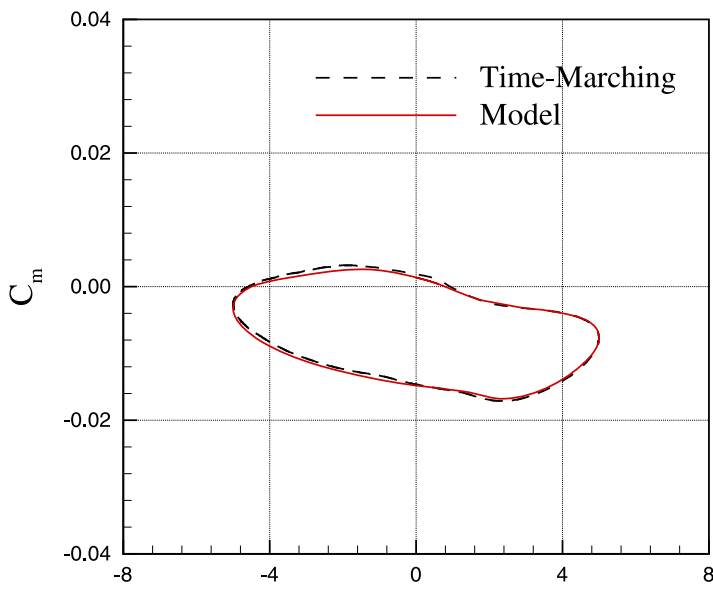

Angle of attack, $\alpha$ (deg)

$\alpha=5^{\circ} \sin (\omega t), k=0.025, \mathrm{M}=0.78$

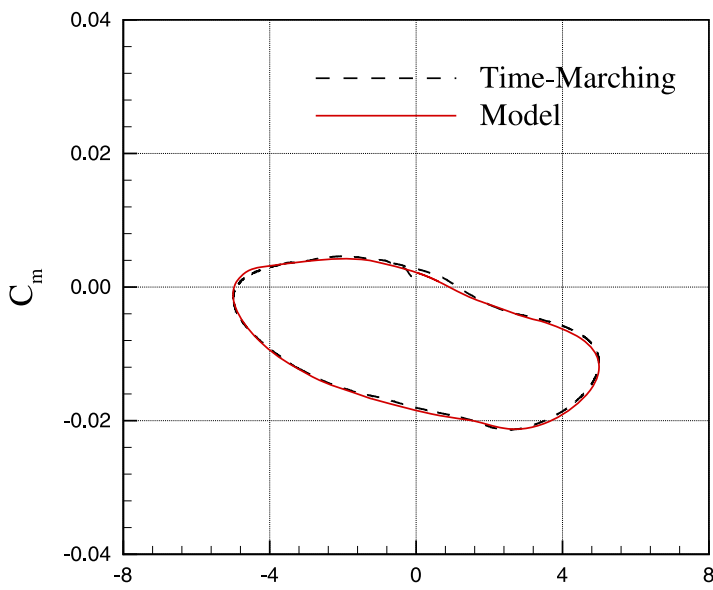

Angle of attack, $\alpha$ (deg)

$\alpha=5^{\circ} \sin (\omega t), k=0.03, \mathrm{M}=0.825$

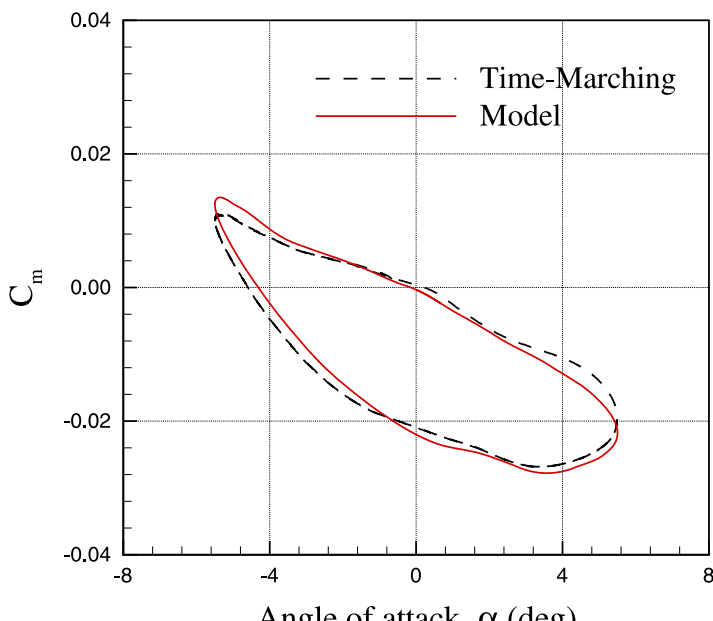

$\alpha=5.5^{\circ} \sin (\omega t), k=0.025, \mathrm{M}=0.88$

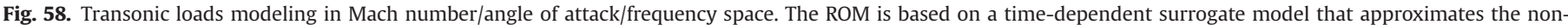
linear indicial functions at different flight conditions. In above $\omega$ is angular velocity and $k=\omega c / 2 V$ is reduced frequency. 
limited number of samples. These responses are a function of time and include the effects of input time derivatives, therefore they can be used for any frequency of interest. Models based on response functions can predict the initial transient behavior seen in the CFD solutions. However, these models are limited to weakly nonlinear systems.

An indirect method was described to estimate the nonlinear Volterra kernels from time-accurate computational fluid dynamic simulations of some special training maneuvers. These maneuvering simulations were also used to estimate the unknown parameters in a model based on RBF. The results showed that both model predictions largely depend on the training maneuver type. A Design of Experiment method was used to generate several pitching motions at different amplitudes and free-stream Mach numbers. The model based on a SBRF then approximated the aerodynamic responses induced by pitching motions at new amplitudes and Mach numbers. However, this model is usually more expensive than other models to generate. Overall, the reduced order models were found to produce accurate results for low to moderate angles of attack and at reasonable computational cost.

\section{Acknowledgments}

Mehdi Ghoreyshi is supported by USAFA under Contract FA70001320018; their financial support is gratefully acknowledged. Acknowledgements are expressed to the DoD High Performance Computing Modernization Program (HPCMP) for providing computer time. The SACCON and X-31 geometries and experimental data were provided by the NATO RTO Task Group AVT-161 on Assessment of Stability and Control Prediction Methods for NATO Air \& Sea Vehicles. The authors appreciate the support provided by the High Performance Computing Research Center at USAFA.

\section{References}

[1] Shilu C, Xiaofei X. Parameter identification of unsteady aerodynamic forces. AIAA paper 92-4505; August 1992.

[2] Pamadi BN. Performance, stability, dynamics, and control of airplanes. AIAA education series, AIAA, Reston, Virginia; 2004

[3] Myatt JH, Schumacher CJ, McKeehen PD, Buffington JD. Modeling and simulation of unsteady aerodynamic effects on a tailless aircraft. AIAA paper 98-4455; August 1998

[4] Abramov NB, Goman MG, Greenwell DI, Khrabrov AN. Two-step linear regression methods for identification of high incidence unsteady aerodynamic model. AIAA paper 2001-4080; August 2001.

[5] Zhang W, Wang B, Ye Z, Quan J. Efficient method for limit cycle flutter analysis by nonlinear aerodynamic reduced-order models. AIAA J 2012;50 (5):1019-28.

[6] Venkatesan C, Friedmann PP. New approach to finite-state modeling of unsteady aerodynamics. AIAA J 1986;24(12):1889-97.

[7] Kyle H, Lowenberg M, Greenwell D. Comparative evaluation of unsteady aerodynamic modeling approaches. AIAA paper 2004-5272; August 2004

[8] Greenwell DI. A review of unsteady aerodynamic modelling for fligh dynamics of manoeuvrable aircraft. AIAA paper 2004-5276; August 2004

[9] Lucia DJ, Beran PS, Silva WA. Reduced-order modeling: new approaches for computational physics. Prog Aerosp Sci 2004;40(1):51-117.

[10] Silva WA. Application of nonlinear systems theory to transonic unsteady aerodynamics responses. J Aircr 1993;30(5):660-8.

[11] Schütte A, Einarsson G, Raichle A, Schoning B, Mönnich W, Forkert T. Numerical simulation of manoeuvreing aircraft by aerodynamic, flight mechanics, and structural mechanics coupling. J Aircr 2009;46(1):53-64.

[12] Ghoreyshi M, Vallespin D, Da Ronch A, Badcock KJ, Vos J, Hitzel S. Simulation of aircraft manoeuvres based on computational fluid dynamics. AIAA paper 2010-8239; August 2010.

[13] Ghoreyshi M, Jirásek A, Cummings RM. CFD modeling for trajectory predic tions of a generic fighter configuration. AIAA paper 2011-6523; August 2011

[14] Sitaraman J, Baeder JD. Computational-fluid-dynamics-based enhanced indicial aerodynamic models. J Aircr 2004;41(4):798-810.

[15] Chin S, Lan CE. Fourier functional analysis for unsteady aerodynamic modeling. AIAA J 1992;30(9):2259-66.
[16] Liebe R. Flow phenomena in nature: a challenge to engineering design. Great Britain: WIT Press: 2007.

[17] Stark VJE. Indicial coefficients for a cropped delta wing in incompressible flow. J Aircr 1985;23(5):370-5.

[18] Lisandrin P, Carpentieri G, van Tooren M. Investigation over CFD-based models for the identification of nonlinear unsteady aerodynamics responses. AIAA J 2006;44(9):2043-50.

[19] McDaniel DR, Cummings RM, Bergeron K, Morton SM, Dean JP. Comparisons of CFD solutions of static and maneuvering fighter aircraft with flight test data. In: 3rd international symposium on integrating CFD and experiments in aerodynamics, U.S. Air Force Academy, Air Force Academy, CO; June 2007.

[20] Raveh DE. Reduced-order models for nonlinear unsteady aerodynamics. AIAA J 2001;39(8):1417-29.

[21] Tischler MB. Aircraft and rotorcraft system identification: engineering methods with flight test examples. AIAA education series, Reston, Virginia; 2006.

[22] Silva W. Identification of noc systems based on the Volterra theory: progress and opportunities. Nonlinear Dyn 2005;39(1-2):25-62.

[23] Marzocca P, Librescu L, Kim D, Lee I, Schober S. Generalized transonic unsteady aerodynamics via computational-fluid-dynamics/indicial approach. AIAA J 2005;43(4):915-7.

[24] Ballhaus WF, Goorjian PM. Computation of unsteady transonic flows by the indicial method. AIAA J 1978;16(2):117-24.

[25] Tobak M. Chapman GT, Schiff LB. Mathematical modeling of the aerodynamic characteristics in flight dynamics. NASA TN-85880; 1984.

[26] Tobak M, Chapman GT. Nonlinear problems in flight dynamics involving aerodynamic bifurcations. NASA TN-86706; 1985.

[27] Ghoryeshi M, Jirásek A, Cummings RM. Computational investigation into the use of response functions for aerodynamic loads modeling. AIAA J 2012;50 (6):1314-27.

[28] Hall KC, Thomas JP, Dowell EH. Reduced-order modelling of unsteady smalldisturbance using a frequency-domain proper orthogonal decomposition technique. AIAA paper 1999-655; January 1999.

[29] Willcox K, Peraire J. Balanced model reduction via the proper orthogonal decomposition. AIAA J 2002:40(11):2323-30.

[30] Silva WA, Bartels RE. Development of reduced-order models for aeroelastic analysis and flutter prediction using the CFL3Dv6.0 code. J Fluids Struct 2004;19(6):729-45.

[31] Silva WA. Discrete-time linear and nonlinear aerodynamic impulse responses for efficient CFD analysis [Ph.D. dissertation]. VA: Faculty of the Department of Applied Science, The College of William and Mary in Virginia; September 1997.

[32] Da Ronch A, McCracken A, Badcock KJ, Ghoreyshi M, Cummings RM. Modeling unsteady aerodynamic loads. AIAA paper 2011-6524; August 2011.

[33] Glaz B, Liu L, Friedmann PP. Reduced-order nonlinear unsteady aerodynamic modeling using a surrogate-based recurrence framework. AIAA J 2010;48 (10):2418-29.

[34] Ghoreyshi M, Cummings RM, Da Ronch A, Badcock KJ. Transonic aerodynamic loads modeling of X-31 aircraft pitching motions. AIAA J, also available as AIAA paper 2012-3127, in press.

[35] Jirásek A, Jeans TL, Martenson M, Cummings RM, Bergeron K. Improved methodologies for maneuver design of aircraft stability and control simulations. AIAA paper 2010-515; January 2010.

[36] Ghoreyshi M, Jirásek A, Cummings RM. Computational approximation of nonlinear unsteady aerodynamics using an aerodynamic model hierarchy. Aerosp Sci Technol 2013;28(1):176-90.

[37] Cummings RM, Jirásek A, Ghoreyshi M, Post ML. Numerical prediction of stability derivatives with various reduced order models. AVT-189 specialists meeting on assessment of stability and control prediction methods for NATO air and sea vehicles, Portsmouth, England; October 2011.

[38] Goman MG, Khrabov AN. State-space representation of aerodynamic characteristics of an aircraft at high angles of attack. AIAA paper 92-4651; August 1992.

[39] Kyle H, Lowenberg M, Greenwell D. Comparative evaluation of unsteady aerodynamic modeling approaches. AIAA paper 2004-5272; August 2004

[40] Ashley H. Some recent developments in interference theory for aeronautical applications. MIT report; July 1963.

[41] Whitehead DS. Force and moment coefficients for vibrating aerofoils in cascade. Aeronautical Research Council Report and Memoranda, Report No. 3254; 1962.

[42] Ashley H, Brunelle E, Moser HH. Unsteady flow through helicopter rotors. Z Angew Math Phys 1958;9(5):57-80.

[43] Miller RH. Rotor blade harmonic air loading. AIAA J 1964;2(7):1254-69.

[44] Daughaday H, Piziali RA. An improved computational model for predicting the unsteady aerodynamic loads of rotor blades. J Am Helicopter Soc 1966;11 (41):3-10.

[45] Hall KC, Ekici K, Thomas JP, Dowell EH. Harmonic balance methods applied to computational fluid dynamics problems. Int J Comput Fluid Dyn 2013;27 (2):52-67.

[46] Sicot F, Guédeney T, Dufour G. Time-domain harmonic balance method for aerodynamic and aeroelastic simulations of turbomachinery flows. Int J Comput Fluid Dyn 2013;27(2):68-78.

[47] McCracken A, Da Ronch A, Timme S, Badcock KJ. Solution of linear systems in Fourier-based methods for aircraft applications. Int J Comput Fluid Dyn 2013;27(2):79-87. 
[48] Vilmina S, Lorrain E, Tartinville B, Capron A, Hirsch C. The nonlinear harmonic method: from single stage to multi-row effects. Int J Comput Fluid Dyn 2013;27(2):88-99.

[49] Gerolymos GA. Filtered chorochronic interface as a capability for 3-D unsteady throughflow analysis of multistage turbomachinery. Int J Comput Fluid Dyn 2013;27(2):100-17.

[50] He L. Fourier spectral modelling for multi-scale aero-thermal analysis. Int J Comput Fluid Dyn 2013;27(2):118-29.

[51] He L. Fourier methods For turbomachinery applications. Prog Aerosp Sci 2010;46(1):329-41.

[52] Dean J, Clifton J, Bodkin D, Morton S, McDaniel D. Determining the applicability and effectiveness of current CFD methods in store certification activities. AIAA paper 2010-1231; January 2010.

[53] Morton S, McDaniel D, Sears D, Tillman B, Tuckey T. Rigid, maneuvering, and aeroelastic results for Kestrel - a CREATE simulation tool. AIAA paper 20101233; January 2010.

[54] Lillian C, Morton S, McDaniel D. Aircraft loads characteristics determined by system and proper orthogonal decomposition of CFD simulations. AIAA paper 2010-4368; July 2010.

[55] Dean J, Morton S, McDaniel D, Clifton J, Bodkin D. Aircraft stability and control characteristics determined by system identification of CFD simulations. AIAA paper 2008-6378; August 2008.

[56] Morelli EA. System IDentification programs for AirCraft (SIDPAC). AIAA paper 2002-4704; August 2002.

[57] Skujins T, Cesnik CES. Toward an unsteady aerodynamic ROM for multiple Mach regimes. AIAA paper 2012-1708; April 2012.

[58] Skujins T, Cesnik CES. Reduced-order modeling of hypersonic unsteady aerodynamics due to multi-modal oscillations. AIAA paper 2011-2341; April 2011.

[59] Skujins T, Cesnik CES. Reduced-order modeling of hypersonic vehicle unsteady aerodynamics. AIAA paper 2010-8127; August 2010.

[60] Glaz B, Liu L, Friedmann PP. A surrogate based approach to reduced-order dynamic stall modeling. AIAA paper 2010-3042; April 2010.

[61] Hall KC, Kielb RE, Ekici K, Thomas JP. Recent advancements in turbomachinery aeroelastic design analysis. AIAA paper 2005-14; January 2005.

[62] Thomas JP, Dowell EH, Hall KC. Three-dimensional transonic aeroelasticity using proper orthogonal decomposition based reduced order models. AIAA paper 2001-1526; April 2001.

[63] Balajewicz M. Reduced order modeling of transonic flutter and limit cycle oscillations using the pruned Volterra series. AIAA paper 2010-2725; April 2010.

[64] Balajewicz M, Dowell E. Reduced-order modeling of flutter and limit-cycle oscillations using the sparse Volterra series. J Aircr 2012;49(6):1803-12.

[65] Lieu T, Farhat C. Adaptation of aeroelastic reduced-order models and application to an F-16 configuration. AIAA J 2007;45(6):1244-57.

[66] Jameson A, Alonso J, McMullen M. Application of a non-linear frequency domain solver to the Euler and Navier-Stokes equations. AIAA paper 200213625; January 2002.

[67] McMullen M, Jameson A, Alonso J. Acceleration of convergence to a periodic steady state in turbomachinery flows. AIAA paper 2001-0152; January 2001.

[68] Woodgate MA, Badcock KJ. Implicit harmonic balance solver for transonic flow with forced motions. AIAA J 2009;47(4):893-901.

[69] Da Ronch A, McCracken AJ, Badcock KJ, Widhalm M, Campobasso MS. Linear frequency domain and harmonic balance predictions of dynamic derivatives. J Aircr 2013:50(3):694-707.

[70] Olcer FE, Prasad JVR, Sankar LN, Bain JJ, Zhao J, He C. Development and evaluation of reduced order models of on-blade control for integrated flight and rotor control. In: Proceedings of the American Helicopter Society, 66th Annual Forum, Phoenix, Arizona; May 2010.

[71] Marques FD, Anderson J. Identification and prediction of unsteady transonic aerodynamic loads by multi-layer functionals. J Fluids Struct 2011;1 (6):83-106

[72] Faller WE, Schreck SJ. Neural networks: applications and opportunities in aeronautics. Prog Aerosp Sci 1996;32(5):433-56.

[73] Faller WE, Schreck SJ. Real-time prediction of unsteady aerodynamics: application for aircraft control and maneuverability enhancement. IEEE Trans Neural Netw 1995;6(6):1461-8.

[74] Jose AI, Baeder JD. Steady and unsteady aerodynamic modeling of trailing edge flaps with overhang and gap using CFD and lower order models. AIAA paper 2009-1071; January 2009.

[75] Leishman JG. Subsonic unsteady aerodynamics caused by gusts using the indicial method. J Aircr 1996;33(5):869-79.

[76] Leishman JG, Nguyen KQ. State-space representation of unsteady airfoil behavior. AIAA J 1990;28(5):836-44.

[77] Raveh DE. CFD-based gust response analysis of free elastic aircraft. AIAA paper 2009-2539; May 2009.

[78] Zaide A, Raveh DE. Numerical simulation and reduced-order modeling of airfoil gust response. AIAA paper 2005-5128; June 2005

[79] Beran P, Stanford B, Kurdi M. Sensitivity analysis for optimization of dynamic systems with reduced order modeling. AIAA paper 2010-1503; January 2010.

[80] Murman SM, Chan WM, Aftosmis MJ, Meakin RL. An interface for specifying rigid-body motions for CFD applications. AIAA paper 2003-1237; January 2003.

[81] Murman SM, Aftosmis MJ, Berger MJ. Simulations of 6-DOF Motion with a Cartesian method. AIAA paper 2003-1246; January 2003.
[82] Korenberg MJ, Hunter IW. The identification of nonlinear biological systems: Volterra kernel approaches. Ann Biomed Eng 1996:24:250-68.

[83] Theodorsen T. General theory of aerodynamic instability and the mechanism of flutter. NACA Technical report 496; 1935.

[84] Sears WR, von Karman T. Airfoil theory for non-uniform motion. J Aeronaut Sci 1938;5(10):379-90.

[85] Küssner HG. Zusammenfassender Bericht Über den Instationaren Auftrieb von Flügeln. Luftfahrtforschung 1939;13(12):410-24.

[86] Sears WR. Some aspects of non-stationary airfoil theory and its practical applications. J Aeronaut Sci 1941;8(1):104-8.

[87] Wagner H. Über die Entstehung des dynamischen Auftriebes von Tragflügeln. Z Angew Math Mech 1925; 1(Book 1):17-35.

[88] Wright JR, Cooper JE. Introduction to aircraft aeroelasticity and loads. UK: John Wiley \& Sons Ltd.; 2007.

[89] Lomax H. Indicial aerodynamics. AGARD manual of aeroelasticity, part 2; October 1960 [chapter 6].

[90] Hodges DH, Pierce GA. Introduction to structural dynamics and aeroelasticity. Cambridge University Press; 2002.

[91] Chen X, Kareem A. Advances in modeling aerodynamic forces on bridge decks. J Eng Mech 2002;128(1):1193-205.

[92] Volterra V. Theory of functionals. London: Blackie; 1930.

[93] Silva WA. Identification of linear and nonlinear aerodynamic impulse responses using digital filter techniques. NASA Technical report 112872 Hampton, Virginia; August 1997.

[94] Wray J, Green GR. Calculation of the Volterra kernels of non-linear dynamic systems using an artificial neural network. Biol Cybern 1994;71:187-95.

[95] Sechi F, Bujatti M. Solid-state microwave high-power amplifiers. Norwood, MA, USA: Artech House INC; 2009.

[96] Wiener N. Nonlinear problems in random theory. Cambridge, MA: MIT Press; 1958.

[97] Barrett JF. The use of functionals in the analysis of non-linear physical systems. J Electr Control 1963;15:567-615.

[98] Kalman RE. Pattern recognition properties of multilinear response functions. J Control Cybern 1980;9:5-31.

[99] Stark L. Neurological control system: studies in bioengineering. New York: Plenum Press; 1968.

[100] Weiner DD, Spina JF. Sinusoidal analysis and modeling of weakly nonlinear circuits. New York: Van Nostrand Reinhold; 1980.

[101] Hunter IW, Korenberg MJ. The identification of nonlinear biological systems: Wiener and Hammerstein cascade models. J Biol Cybern 1986;55:135-44.

[102] Korenberg MJ. A fast orthogonal search method for biological time-series analysis and system identification. In: Proceedings of IEEE international conference on systems, man, and cybernetics; 1989. p. 459-65.

[103] Korenberg MJ. A robust orthogonal for system identification and time-series analysis. J Biol Cybern 1989;60:267-76.

[104] Jirásek A, Cummings RM. Reduced order modeling of X-31 wind tunne model aerodynamic loads. Aerospace Science and Technology 2012;20 (1):52-60.

[105] Balajewicz M, Nitzsche F, Feszty D. Application of multi-input Volterra theory to nonlinear multi-degree-of-freedom aerodynamic systems. AIAA J 2010;48 (1):56-62.

[106] Leishman JG. Indicial lift approximations for two-dimensional subsonic flow as obtained from oscillatory measurements. J Aircr 1993;30(3):340-51.

[107] Duffy DG. Advanced engineering mathematics with MATLAB. second ed. Florida: Chapman and Hall, CRC; 2003.

[108] Reisenthel PH. Development of a nonlinear indicial model using response functions generated by a neural network. AIAA paper 97-0337; January 1997

[109] Reisenthel PH, Bettencourt MT. Data-based aerodynamic modeling using nonlinear indicial theory. AIAA paper 97-0337; January 1999.

[110] Findeisen D. System dynamics and mechanical vibrations: an introduction. Berlin: Springer-Verlag; 2000.

[111] Roskam J. Airplane design. Kansas, USA: Roskam Aviation and Engineering Corporation; 1990.

[112] Ghoreyshi M, Cummings RM. Unsteady aerodynamics modeling for aircraft maneuvers: a new approach using time-dependent surrogate modeling. AIAA paper 2012-3327; June 2012.

[113] Babuska R. Fuzzy modeling for control. Norwell, USA: Kluwer Academic Publishers Group; 1998.

[114] Arbib MA. The handbook of brain theory and neural networks. second edThe MIT Press; 2003.

[115] Levin AU, Narendra KS. Control of nonlinear dynamical systems using neural networks: controllability and stabilization. IEEE Trans Neural Netw 1993;4 (2):192-206.

[116] Krantz SG, Parks HR. The implicit function theorem: history, theory, and applications. Boston: Birkhauser; 2002.

[117] Da Ronch A, Ghoreyshi M, Badcock KJ. On the generation of flight dynamics aerodynamic tables by computational fluid dynamics. Prog Aerosp Sci 2011;47:597-620.

[118] Jategaonkar RV. Flight vehicle system identification. AIAA educational series, vol. 216, Reston, VA; 2006.

[119] George DA. Continuous nonlinear systems. Technical report 335. Cambridge, Massachusetts: M.I.T. Research Laboratory of Electronics; 1959.

[120] Schetzen M. Measurement of the kernels of a nonlinear system of finite order. Int J Control 1965;1:251-63. 
[121] Milanece A, Marzocca P. Volterra kernels identification using continuous time impulses applied to a nonlinear aeroelastic problem. AIAA paper 2009-2321; May 2009.

[122] Tromp J, Jenkins JE. A Volterra kernel identification scheme applied to aerodynamic reactions. AIAA paper 1990-2803; August 1990

[123] Bisplinghoff RL, Ashley H, Ralfman RL. Aeroelasticity. Mineola, NY: Dover Publications; 1996

[124] Garrick IE, H RW. Historical development of aircraft flutter. J Aircr 1981;18 (11):897-912.

[125] Librescu L, Song O. Thin-walled composite beams: theory and application. Dordrecht, Netherland: Springer; 2006.

[126] Mazelsky B, Drischler JA. Numerical determination of indicial lift and moment functions for a two-dimensional sinking and pitching airfoil at mach number 0.5 and 0.6. NACA technical note, 2739; 1952.

[127] Dowell EH. A simple method for converting frequency domain aerodynamics to the time domain. NASA report TM-81844; 1980.

[128] Magnus RJ. Calculation of some unsteady transonic flows about the NACA 64006 and 64A010 airfoils. AFFDL-TR-77-46; July 1977.

[129] Singh R, Baeder JD. Direct calculation of three-dimensional indicial lift response using computational fluid dynamics. J Aircr 1997;34(4):465-71.

[130] Ueda T, Dowell EH. Flutter analysis using nonlinear aerodynamic forces. AIAA J 1984;21(2):101-9.

131] Ghoreyshi M, Badcock KJ, Woodgate MA. Accelerating the numerical generation of aerodynamic models for flight simulation. I Aircr 2009:46 (3):972-80.

[132] Ghoryeshi M, Post ML, Cummings RM. CFD calculation of aerodynamic indicial functions for a generic fighter configuration. ITEA J Test Eval 2012;33(6):348-64.

[133] Görtz S, McDaniel D, Morton SA. Towards an efficient aircraft stability and control analysis capability using high-fidelity CFD. AIAA paper 2007-1053; January 2007.

[134] O'Neill C, Arena A. Time domain training signals comparison for computational fluid dynamics based aerodynamic identification. J Aircr 2005;42 (2):421-8.

[135] Murphy PC, Klein V. Validation of methodology for estimating aircraft unsteady aerodynamics parameters from dynamic wind tunnel tests. AIAA paper 2003-5397; August 2003.

[136] Morelli EA. Real time parameters estimation in the frequency domain. AIAA paper 99-4043; August 1999.

[137] Strang WZ, Tomaro RF, Grismer MJ. The defining methods of cobalt: a parallel, implicit, unstructured Euler/Navier-Stokes flow solver. AIAA paper 1999-0786; 1999.

[138] Gottlieb JJ, Groth CPT. Assessment of Riemann solvers for unsteady onedimensional inviscid flows of perfect gasses. I Fluids Struct 1998;78 (2):437-58.

[139] Tomaro RF, Strang WZ, Sankar LN. An implicit algorithm for solving time dependent flows on unstructured grids. AIAA paper 1997-0333; 1997.

[140] Spalart PR, Allmaras SR. A one equation turbulence model for aerodynamic flows. AIAA paper 1992-0439; January 1992

[141] Wilcox DC. Reassessment of the scale determining equation for advanced turbulence models. AIAA J 26 November 1988; 1299-310.

[142] Menter F. Eddy viscosity transport equations and their relation to the $k-\varepsilon$ model. ASME J Fluids Eng 1997;119:876-84.

[143] Tyssel L. Hybrid grid generation for complex 3D geometries. In: Proceedings of the 7th international conference on numerical grid generation in computational field simulation; 2000. p. 337-46.
[144] Tyssel L. The TRITET grid generation system. International Society of Grid Generation (ISGG). In: Proceedings of the 10the international conference on numerical grid generation in computational field simulations; 2000.

[145] Ladson CL. Effects of independent variation of Mach and Reynolds number on the low-speed aerodynamic characteristics of NACA0012 airfoil section. NASA technical paper 4074; January 1988.

[146] Landon RH. NACA 0012. Oscillating and transient pitching, in compendium of unsteady aerodynamic measurements. AGARD-R-702, Data Set 3; August 1982.

[147] Beyers ME. Subsonic roll oscillation experiments on the standard dynamic model. AIAA paper 83-2134; August 1983.

[148] Jermey C, Schiff LB. Wind tunnel investigation of the aerodynamic characteristics of the standard dynamic model in coning motion at Mach 0.6. In: 12th AIAA atmospheric flight mechanics conference, AIAA Paper 1985-1828; 1985.

[149] Balakrishna S, Niranjana T. Wind tunnel dynamic flying study of the pitching moment derivatives of the standard dynamics model in active control. AIAA paper 87-2626; August 1987.

[150] Huang XZ. Wing and fin buffet on the standard dynamic model. RTO Technical report RTO-TR-26; October 2000.

[151] Cummings RM, Schütte A. Integrated computational/experimental approach to unmanned combat air vehicle stability and control estimation. J Aircr 2012;49(6):1542-57

[152] Löeser T, Vicroy D, Schütte, A. SACCON static wind tunnel tests at DNW-NWB and $14^{\prime} \times 22^{\prime}$ NASA LaRC. AIAA paper 2010-4393; June-July 2010.

[153] Spalart PR, Schur M. On the sensitisation of turbulence models to rotation and curvature. Aerosp Sci Technol 1997;1:297-302.

[154] Canter DE, Groves AW. X-31 post-stall envelope expansion and tactical utility testing. AIAA paper 1994-2171; June 1994

[155] Alcorn CW, Croom MA, Francis MS. The X-31 experience-aerodynamic impediments to post-stall agility. AIAA paper 1995-362; January 1995.

[156] Williams DL, Nelson RC, Fisher D. An investigation of X-31 roll characteristics at high angle-of-attack through subscale model testing. AIAA paper 1994806; January 1994.

[157] Rein M, Höhler G, Schütte A, Bergmann A, Löeser T. Ground-based simulation of complex maneuvers of a delta-wing aircraft. AIAA paper 2006-3149; June 2006.

[158] Schefter J. X-31: how they're inventing a radical new way to fly. Pop. Sci. 1989;234(2):58-64.

[159] Ghoreyshi M, Jirásek A, Cummings RM. Challenges in the aerodynamics modeling of an oscillating and translating airfoil at large incidence angles. Aerosp Sci Technol 2013;28(1):133-44.

[160] Mackman TJ, Allen CB, Ghoreyshi M, Badcock KJ. Comparison of adaptive sampling methods for generation of surrogate aerodynamic models. AIAA J 2013;51(4):797-808

[161] Da Ronch A, Vallespin D, Ghoreyshi M, Badcock KJ. Evaluation of dynamic derivatives using computational fluid dynamics. AIAA J 2012;50(2):470-84.

[162] Betts JT. Survey of numerical methods for trajectory optimization. J Guid Control Dyn 1997:20(1):57-60.

[163] Hull DG. Conversion of optimal control problems into parameter optimization problems. J Guid Control Dyn 1998;21(2):193-207.

[164] Love MC. Flight maneuvers. New York, USA: McGraw-Hill; 1999.

[165] Anderson F. Flying the mountains: a training manual for flying single-engine aircraft. New York, USA: McGraw-Hill; 2003

[166] Ross IM, Fahroo F. User's manual for DIDO 2002: a MATLAB application package for dynamic optimization. NPS Technical report. Department of Aeronautics and Astronautics, Naval Postgraduate School, AA-02-002; 2002. 